Portland State University

PDXScholar

Civil and Environmental Engineering Faculty

Publications and Presentations

Civil and Environmental Engineering

$9-2010$

\title{
Characteristics of Transitions in Freeway Traffic
}

Robert L. Bertini

Portland State University

Soyoung Ahn

Arizona State University

Follow this and additional works at: https://pdxscholar.library.pdx.edu/cengin_fac

Part of the Civil and Environmental Engineering Commons, and the Transportation Commons Let us know how access to this document benefits you.

\section{Citation Details}

Bertini, Robert L. and Soyoung Ahn. Characteristics of Transitions in Freeway Traffic. OTREC-RR-10-12. Portland, OR: Transportation Research and Education Center (TREC), 2010. http://dx.doi.org/10.15760/ trec. 150

This Report is brought to you for free and open access. It has been accepted for inclusion in Civil and Environmental Engineering Faculty Publications and Presentations by an authorized administrator of PDXScholar. Please contact us if we can make this document more accessible: pdxscholar@pdx.edu. 


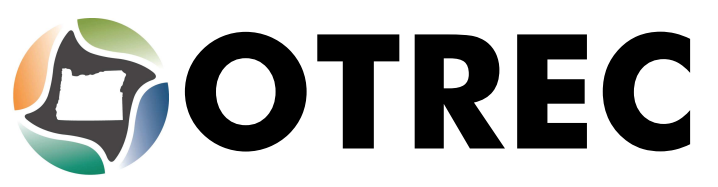

FINAL REPORT

\section{Characteristics of Transitions in Freeway Traffic}

OTREC-RR-10-12

September 2010 



\title{
CHARACTERISTICS OF TRANSITIONS IN FREEWAY TRAFFIC
}

Report Type

OTREC-RR-10-12

\author{
by \\ Robert Bertini \\ Portland State University \\ Soyoung Ahn \\ Arizona State University
}

for

Oregon Transportation Research and Education Consortium (OTREC)

P.O. Box 751

Portland, OR 97207

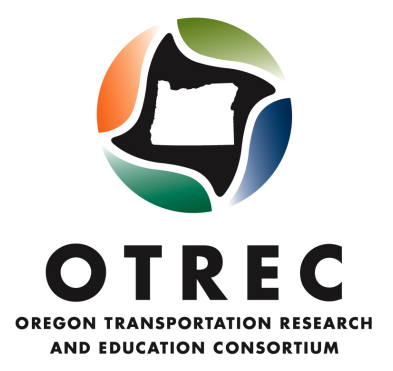

September 2010 


\begin{tabular}{|c|c|c|}
\hline \multicolumn{3}{|c|}{ Technical Report Documentation Page } \\
\hline $\begin{array}{l}\text { 1. Report No. } \\
\text { OTREC-RR-10-12 }\end{array}$ & 2. Government Accession No. & 3. Recipient's Catalog No. \\
\hline \multirow{2}{*}{\multicolumn{2}{|c|}{$\begin{array}{l}\text { 4. Title and Subtitle } \\
\text { CHARACTERISTICS OF TRANSITIONS IN FREEWAY TRAFFIC }\end{array}$}} & $\begin{array}{l}\text { 5. Report Date } \\
\text { September } 2010\end{array}$ \\
\hline & & 6. Performing Organization Code \\
\hline \multicolumn{2}{|c|}{$\begin{array}{l}\text { 7. Author(s) } \\
\text { Robert Bertini, Portland State University } \\
\text { Soyoung Ahn, Arizona State University } \\
\text { Manasa Rayabhari, Arizona State University } \\
\text { Oing Ou, , Delft University of Technology } \\
\text { Wei Feng, Portland State University }\end{array}$} & 8. Performing Organization Report No. \\
\hline \multirow{2}{*}{\multicolumn{2}{|c|}{$\begin{array}{l}\text { 9. Performing Organization Name and Address } \\
\text { Portland State University } \\
\text { Arizona State University }\end{array}$}} & 10. Work Unit No. (TRAIS) \\
\hline & & 11. Contract or Grant No. \\
\hline \multirow{2}{*}{\multicolumn{2}{|c|}{$\begin{array}{l}\text { 12. Sponsoring Agency Name and Address } \\
\text { Oregon Transportation Research } \\
\text { and Education Consortium (OTREC) } \\
\text { P.O. Box } 751 \\
\text { Portland, Oregon } 97207\end{array}$}} & 13. Type of Report and Period Covered \\
\hline & & 14. Sponsoring Agency Code \\
\hline
\end{tabular}

15. Supplementary Notes

\section{Abstract}

This research seeks to understand the characteristics of transitions as freeway traffic changes from one state to another. This study addresses the features of two types of transitions; transitions near a merge and transitions along shock waves during the onsets and dissipations of queues at several freeway sites.

Individual vehicle trajectory data were analyzed for studying the transitions near a merge. The length of a transition zone was measured by analyzing the spatial changes in flow, density and speed along kinematic waves near a merge. It was found that the length of transition in terms of flow, density and speed were respectively around $90 \mathrm{~m}, 120 \mathrm{~m}$ and $180 \mathrm{~m}$ indicating that the transition in flow occurs over a short distance while the transition in speed occurs in much longer space.

The dynamics of the transition zone were explored by analyzing the relationship among the transition durations, rates and various traffic and geometric variables at four freeway sites. Transition durations observed from the four sites vary from 10 to 24 minutes during the onsets of queues while the durations ranged from 10 to 30 minutes during the dissipations of the queues. At each site, formations and dissipations of queues displayed similar durations. Transition rates during the onsets of queues ranged from -7.6 to $-2.2 \mathrm{kmph} / \mathrm{min}$ while they ranged from 2.0 to $6.2 \mathrm{kmph} / \mathrm{min}$ during the dissipations of queues. Some lane-specific features are observed in terms of initial speeds (just prior to transition), change in speed during transition, transition durations, and rates. It is also found that the structure of transition does not change in the absence of freeway interchanges as a queue expands and recedes. Finally, it is found that the transition rates tend to be larger upstream of an on-ramp while they tend to be smaller upstream of an off-ramp, indicating that inflows and outflows have different effects on transition characteristics.

\begin{tabular}{l|l} 
17. Key Words & 18. Distribution Statement
\end{tabular} No restrictions. Copies available from OTREC: www.otrec.us

\begin{tabular}{|l|l|l|l|}
\hline $\begin{array}{l}\text { 19. Security Classification (of this report) } \\
\text { Unclassified }\end{array}$ & $\begin{array}{l}\text { 20. Security Classification (of this page) } \\
\text { Unclassified }\end{array}$ & $\begin{array}{l}\text { 21. No. of Pages } \\
98\end{array}$ & 22. Price \\
\hline
\end{tabular}




\section{ACKNOWLEDGEMENTS}

This research was funded by Oregon Transportation Research and Education Consortium, Arizona State University, and Portland State University. The FHWA provided the NGSIM data, and Stuart Beale of the U.K. Highways Agency generously provided the M4 data. Caltrans, California Path and California Center for Innovative Transportation generously provided the I-80 data.

\section{DISCLAIMER}

The contents of this report reflect the views of the authors, who are solely responsible for the facts and the accuracy of the material and information presented herein. This document is disseminated under the sponsorship of the U.S. Department of Transportation University Transportation Centers Program, Arizona State University and Portland State University in the interest of information exchange. The U.S. Government, Arizona State University and Portland State University assumes no liability for the contents or use thereof. The contents do not necessarily reflect the official views of the U.S. Government, Arizona State University and Portland State University. This report does not constitute a standard, specification, or regulation. 


\section{TABLE OF CONTENTS}

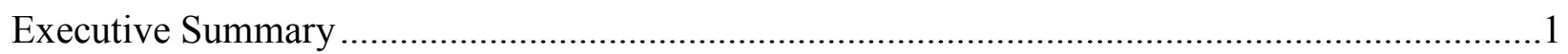

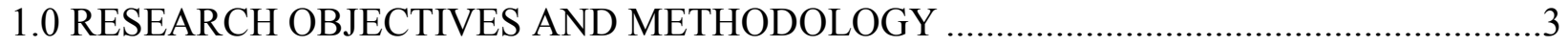

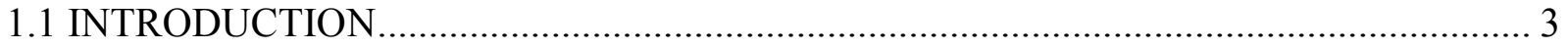

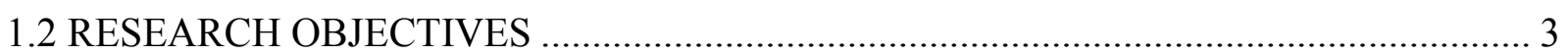

1.2.1 Transition near a Merge ………............................................................................ 3

1.2.2 Transition near the Tail of a Queue ........................................................................... 4

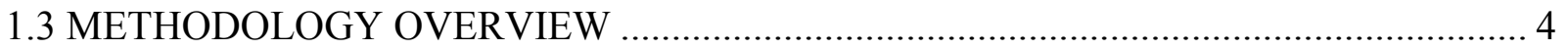

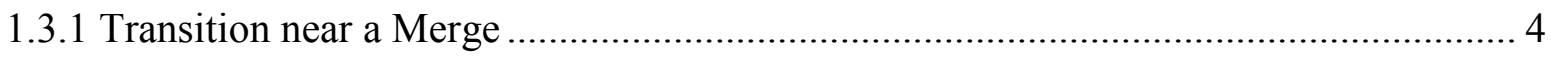

1.3.2 Transition near the Tail of a Queue ………………................................................ 4

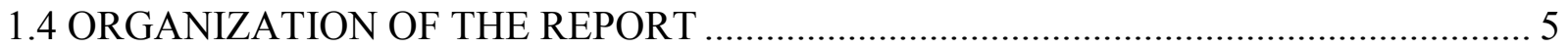

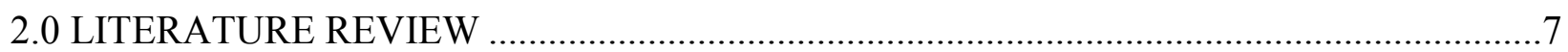

2.1 HISTORY OF TRAFFIC FLOW THEORY …………............................................... 7

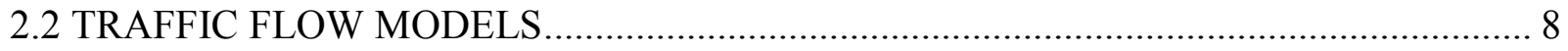

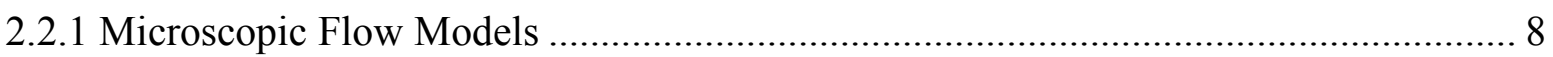

2.2.2 Macroscopic Flow Models ........................................................................................ 9

2.2.2.1 The Continuum Hypothesis - Conservation Law............................................... 10

2.2.2.2 Lighthill-Whitham-Richards Theory: Kinematic Wave Theory.......................... 10

2.2.2.3 Newell's Simplified Kinematic Wave Theory.................................................... 11

2.2.2.4 Empirical Support of First-Order Model......................................................... 13

2.2.2.5 Extensions to the First-Order Model ............................................................... 13

2.2.2.6 Higher-Order Models ............................................................................. 15

2.3 EMPIRICAL STUDIES OF TRANSITION ZONE …………..................................... 15

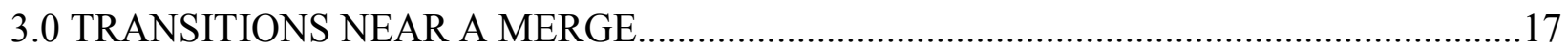

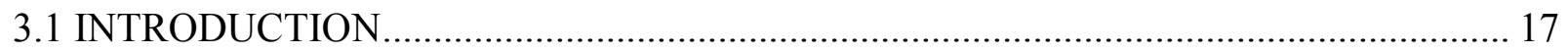

3.2 STUDY SITE AND DATA DESCRIPTION ………………..................................... 17

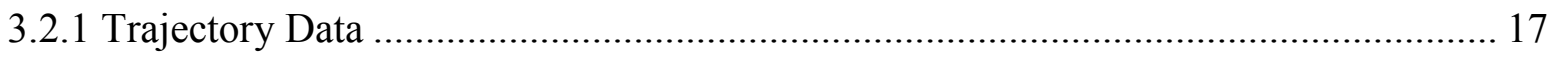

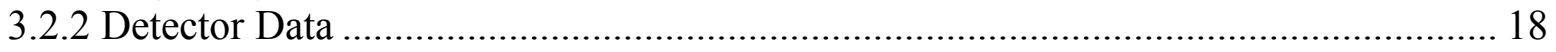

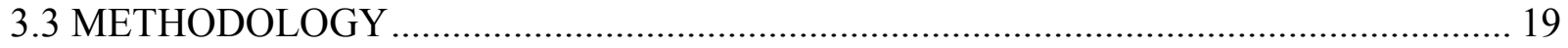

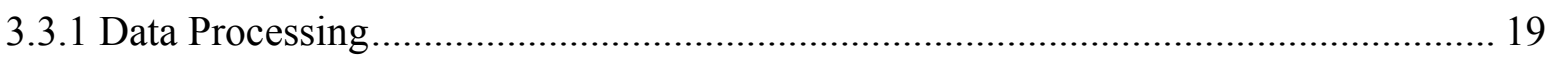

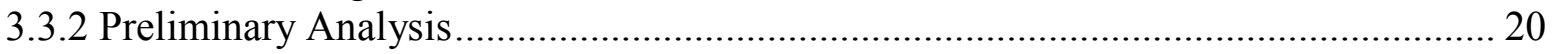

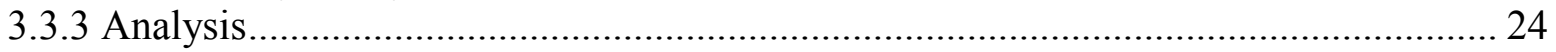

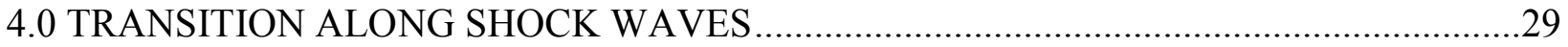

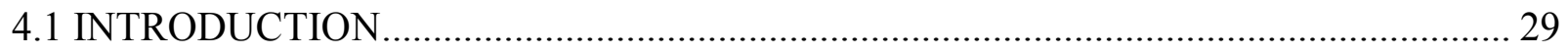




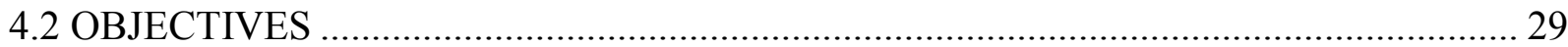

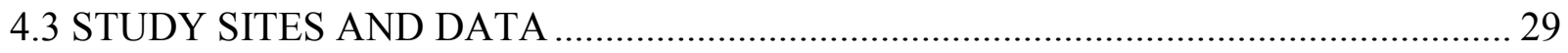

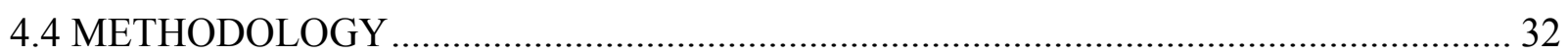

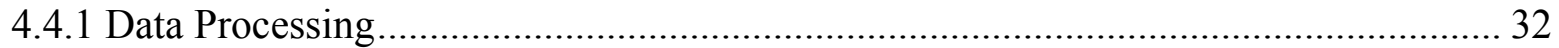

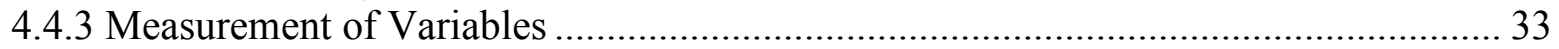

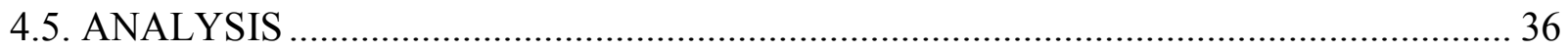

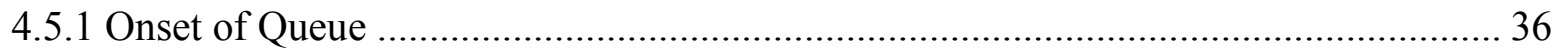

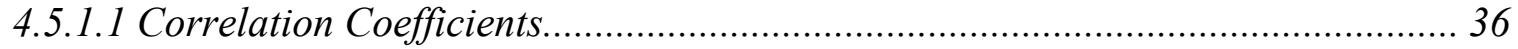

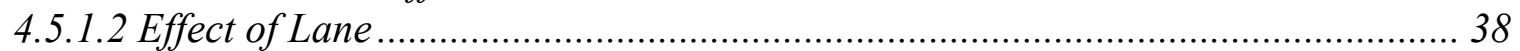

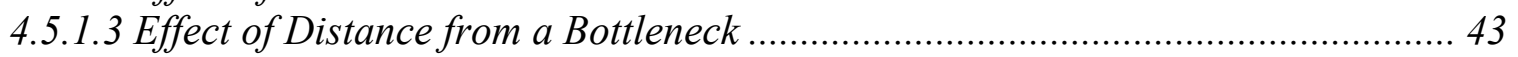

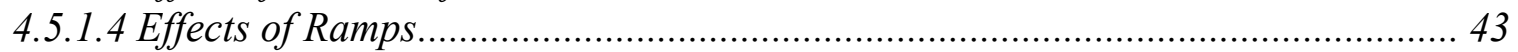

4.5.2 Clearance of Queue ............................................................................................ 44

4.5.2.1 Correlation Coefficients............................................................................. 45

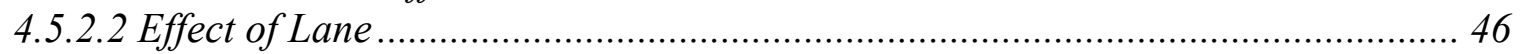

4.5.2.3 Effect of Distance from a Bottleneck .............................................................. 47

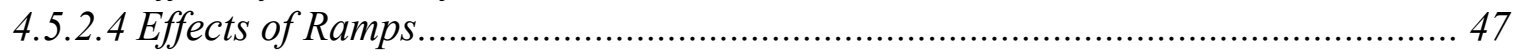

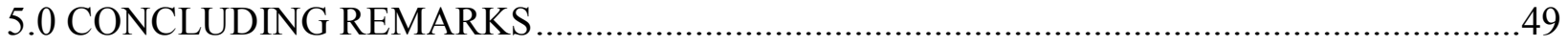

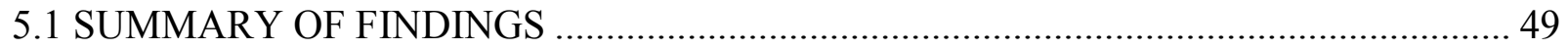

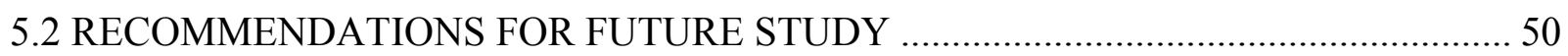

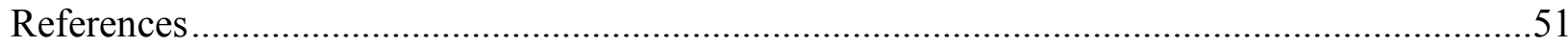

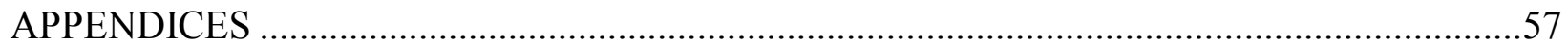

APPENDIX A: OTHER SITES NOT INCLUDED IN THE REPORT ………….........................59

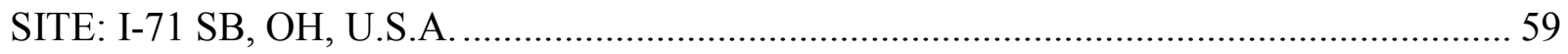

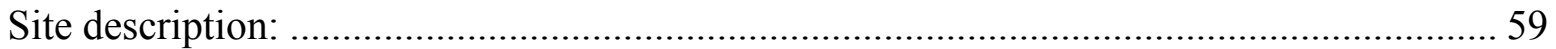

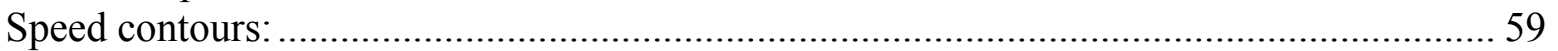

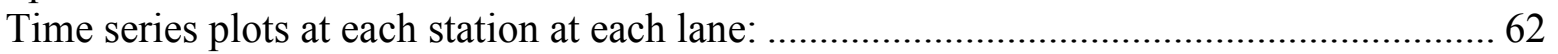

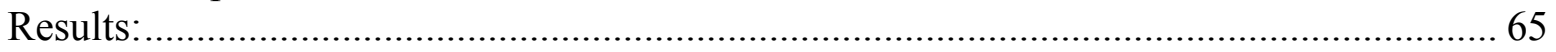

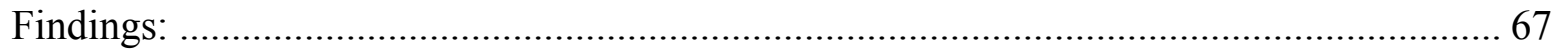

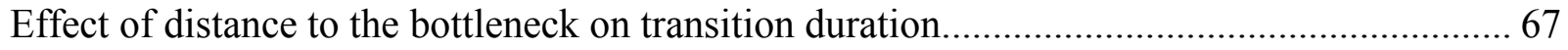

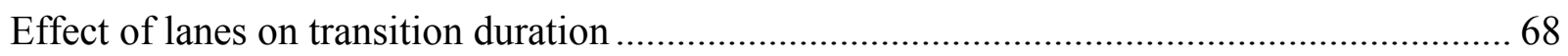

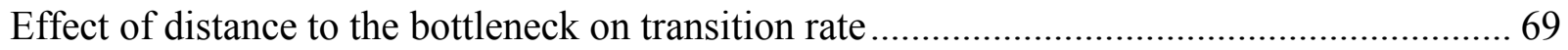

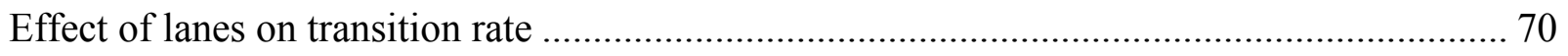

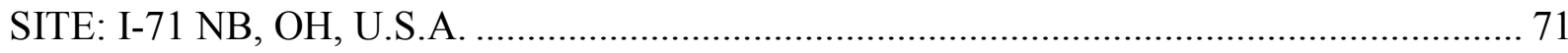

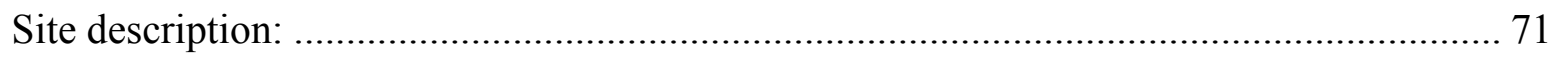




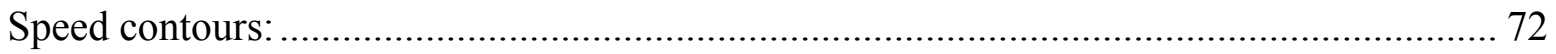

Time series plots at each station at each lane: ........................................................ 77

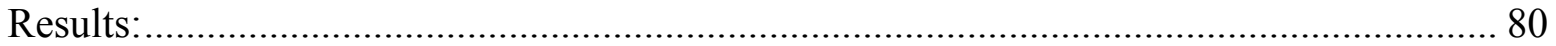

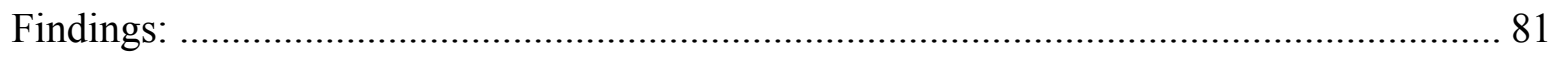

Effect of distance to the bottleneck on transition duration............................................... 81

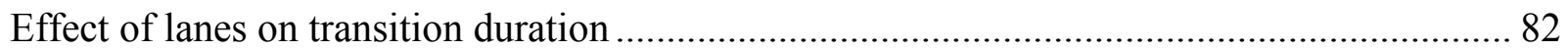

Effect of distance to the bottleneck on transition rate ..................................................... 83

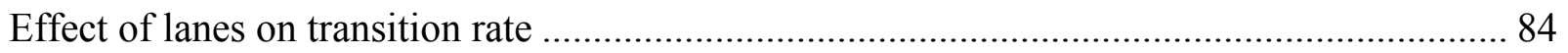

SITE: A2-SB, EVERDINGEN, UTRECHT, HOLLAND.............................................. 86

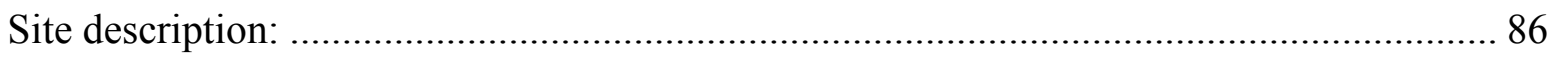

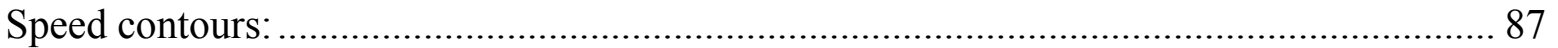

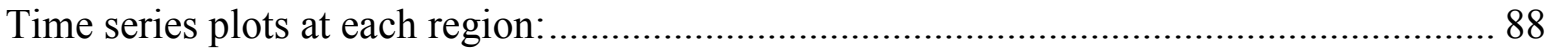

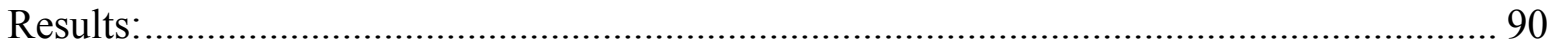

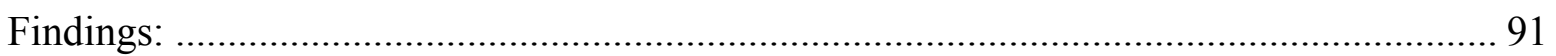

SITE: I-405 NB AT MULHOLLAND DRIVE, LOS ANGELES, CA .............................. 92

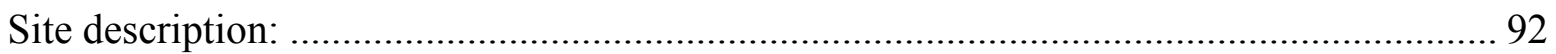

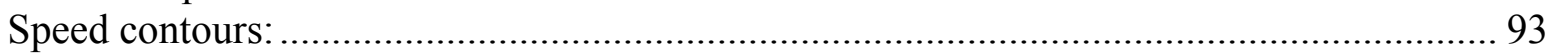

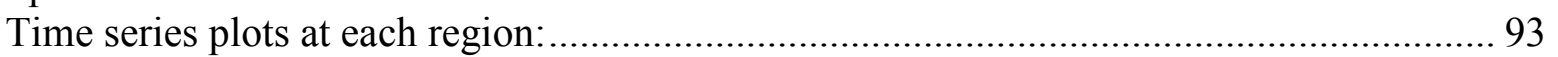

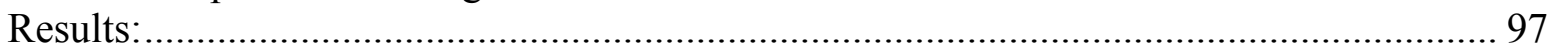

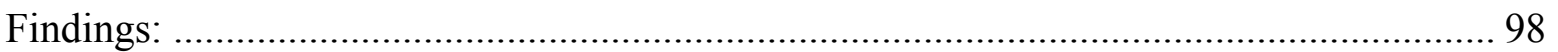

\section{APPENDICES}

APPENDIX A: ANALYSIS OF INDIVIDUAL SITES 


\section{LIST OF TABLES}

Table 3.1: Time Periods of Near-Stationary Traffic States at Section 14 .................................. 22

Table 3.2: Summary of Changes in Traffic Variables and Respective Transition Lengths ........ 25

Table 4.1: Median Transition Durations and Rates at Four Study Sites.................................. 36

Table 4.2: Correlation Coefficients among Explanatory Variables: M4 (Onset) ...................... 37

Table 4.3: Correlation Coefficients among Explanatory Variables: A4 (Onset)....................... 37

Table 4.4: Correlation Coefficients among Explanatory Variables: I-80 (Onset)..................... 37

Table 4.5: Correlation Coefficients among Explanatory Variables: I-5 (Onset) ........................ 37

Table 4.6: Statistical Test Results for the Effect of Lane: M4 (Onset)................................... 39

Table 4.7: Statistical Test Results for the Effect of Lane: A4 (Onset) ..................................... 40

Table 4.8: Statistical Test Results for the Effect of Lane: I-80 (Onset) .................................... 41

Table 4.9: Statistical Test Results for the Effect of Lane: I-5 (Onset) ................................... 42

Table 4.10: Statistical Test Results for the Effect of Loop: M4 (Onset) .................................. 43

Table 4.11: Statistical Test Results for the Effect of Loop: A4 (Onset) ................................... 43

Table 4.12: Median Transition Rates at Loop Stations 5 and 6, I-80 (Onset) ........................... 44

Table 4.13: Median Transition Rates at Loop Stations 2 and 3, I-5 (Onset) ............................ 44

Table 4.14: Median Transition Durations and Rates at Four Study Sites (Clearance)................ 45

Table 4.15: Correlation Coefficients of Explanatory Variables: M4 (Clearance) ...................... 45

Table 4.16: Correlation Coefficients of Explanatory Variables: A4 (Clearance)....................... 45

Table 4.17: Correlation Coefficients of Explanatory Variables: I-80 (Clearance) ..................... 45

Table 4.18: Correlation Coefficients of Explanatory Variables: I-5 (Clearance) ....................... 46

Table 4.19: Statistical Test Results for the Effect of Lane: M4 (Clearance)............................. 46

Table 4.20: Statistical Test Results for the Effect of Lane: A4 (Clearance)............................. 46

Table 4.21: Statistical Test Results for the Effect of Lane: I-80 (Clearance) ........................... 46

Table 4.22: Statistical Test Results for the Effect of Lane: I-5 (Clearance)............................. 47

Table 4.23: Statistical Test Results for the Effect of Loop: M4 (Clearance) ............................ 47

Table 4.24: Statistical Test Results for the Effect of Loop: A4 (Clearance) ............................. 47

Table 4.25: Median Transition Rates at Loop Stations 5 and 6, I-80 (Clearance) .................... 48

Table 4.26: Median Transition Rates at Loop Stations 2 and 3, I-5 (Clearance) ...................... 48 


\section{LIST OF FIGURES}

Figure 3.1: Eastbound Interstate 80 Study Area [Adopted from http://ww.ngsim.fhwa.dot.gov] (a) site schematic; (b) aerial photo; (c) detector locations ..................................................... 18

Figure 3.3: Speed Contours of I-80 Showing Near-Stationary Time-Space Regions $\left(t_{5}\right.$ to $\left.t_{11}\right)$ (a) 4-4:15 p.m. (b) 5-5:30 p.m.

Figure 3.4: Rescaled Cumulative Curves of Space Mean Speed at Section 14 (a) 4-4:15 p.m. (b) 5-5:30 p.m.

Figure 3.5: Evolutions of Traffic Variables over Freeway Sections in Stationary Period, $t_{9}$ (a) Flow vs. freeway section; (b) Density vs. freeway section; (c) space-mean speed vs. freeway section

Figure 3.6: Effects of Traffic Variables on Length of Transition

(a) Length of transition vs. change in flow; (b) Length of transition vs. change in density; (c)

Length of transition vs. change in space-mean speed

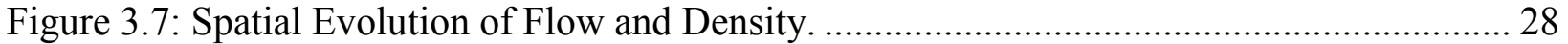

Figure 4.1: Schematic of M4 eastbound, London, U.K........................................................ 30

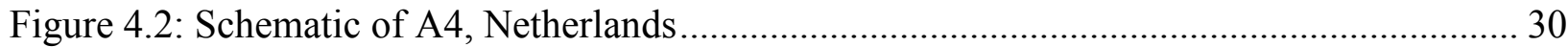

Figure 4.3: Schematic of eastbound I-80, California, U.S.A................................................ 31

Figure 4.4: Schematic of eastbound I-5, Oregon, U.S.A.................................................. 31

Figure 4.5: Example speeds vs. time at I-80, August 6, 2002

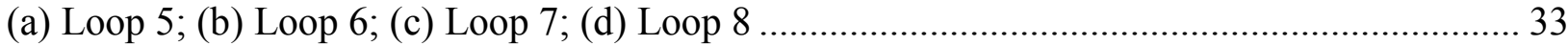

Figure 4.6: Speed contours representing all lanes for M4 ............................................... 33

Figure 4.7: Five-Minute Moving-Average Speeds with Start and End Points of Transition for M4

Figure 4.8: Lane-wise distribution of transition characteristics at onset of queue, M4

(a) Initial speed; (b) Change in speed; (c) Transition Duration; (d) Transition Rate 39

Figure 4.9: Lane-wise distribution of transition characteristics at onset of queue, A4

(a) Initial speed; (b) Change in speed; (c) Transition Duration; (d) Transition Rate 40

Figure 4.10: Lane-Wise Transition Duration Distributions by Detector Station for Onset of Queue, I-80

Figure 4.11: Lane-Wise Transition Duration Distributions by Detector Station for Onset of Queue, I-5

Figure 4.12: Median transition rates at onsets of queues over loop detector stations (a) I-80; (b) I-5

Figure 4.13: Median transition rates at onsets of queues over loop detector stations (a) I-80 (b) I-5 


\section{EXECUTIVE SUMMARY}

This research seeks to understand the characteristics of transitions as freeway traffic changes from one state to another. This study addresses the features of two types of transitions; transitions near a merge and transitions along shock waves during the onsets and dissipations of queues at several freeway sites.

Individual vehicle trajectory data were analyzed for studying the transitions near a merge. The length of a transition zone was measured by analyzing the spatial changes in flow, density and speed along kinematic waves near a merge. It was found that the length of transition in terms of flow, density and speed were respectively around 90 meters, 120 meters and 180 meters. This indicates that the transition in flow occurs over a short distance while the transition in speed occurs in much longer space.

The dynamics of the transition zone were explored by analyzing the relationship among the transition durations, rates and various traffic and geometric variables at four freeway sites. Transition durations observed from the four sites vary from 10 to 24 minutes during the onsets of queues while the durations ranged from 10 to 30 minutes during queue dissipations. At each site, queue formations and dissipations displayed similar durations. Transition rates during the onsets ranged from -7.6 to $-2.2 \mathrm{kmph} / \mathrm{min}$ while they ranged from 2.0 to $6.2 \mathrm{kmph} / \mathrm{min}$ during dissipations. Some lane-specific features are observed in terms of initial speeds (just prior to transition), change in speed during transition, transition durations, and rates. In addition, the transition structure does not change in the absence of freeway interchanges as a queue expands and recedes. Finally, transition rates tend to be larger upstream of an on-ramp and smaller upstream of an off-ramp, indicating that inflows and outflows have different effects on transition characteristics. 


\subsection{RESEARCH OBJECTIVES AND METHODOLOGY}

\subsection{INTRODUCTION}

A transition occurs when vehicles accelerate or decelerate while traffic changes from one (stationary) state to another. A transition takes place over a particular time and distance due to limited acceleration or deceleration capabilities and driving behavior. Traffic states change for various reasons, including demand changes, congestion onset or dissipation, and changes in bottleneck discharge rates and ramp flows. As these are common features of urban freeways, freeway traffic undergoes transitions frequently and rarely exhibits stationary states for a prolonged period of time.

A macroscopic study of transition zones and shock wave propagation was conducted by Muñoz and Daganzo (2003) in order to identify the transition periods for a regime change. They observed that the data points on the flow-occupancy curve moved from the uncongested to congested branches (and vice versa) and the transition zone propagated upstream at the speed predicted by the LWR model. They measured the transition duration at each detector location and found that transition occurred over several minutes. However, the LWR model lacked accuracy due to its instantaneous transitions assumption. Moreover, their study pertained to the "tail of a queue," and the transition zones were traced over a short distance only on a single day. Hence, this research work addresses the shortcomings of Muñoz and Daganzo's (2003) work and provides a larger picture of how transitions occur.

\subsection{RESEARCH OBJECTIVES}

The primary objective of this research is to better understand the characteristics of different types of transitions that freeway traffic undergoes using various data sources available. The study mainly addresses the transitions that occur near a merge and the tail-end of a queue. For each type, the basic characteristics such as transition duration and length, as well as their relationships with other traffic variables, are analyzed. More detailed descriptions of the objectives follow.

\subsubsection{Transition near a Merge}

Transitions occur near a freeway merge as vehicles enter the freeway through an on-ramp and change lanes to reach their destination lanes. Due to the inflow from an on-ramp, the traffic conditions upstream of the merge are characterized by higher densities, lower flows and lower speeds in congestion, compared to the conditions downstream of the merge. This study focuses on the transition zone that separates the conditions upstream and downstream of the merge. In particular, the length of transition is measured in terms of traffic parameters, flow, density and speed. It is anticipated that the changes in these traffic parameters take place gradually over space. 


\subsubsection{Transition near the Tail of a Queue}

The tail of a queue separates a queued state from a freely flowing state. This study aims to analyze the characteristics of traffic transition as the queue grows (i.e., propagates against traffic flow) and then recedes with decreasing demand. The transition duration is traced along the freeway in the direction of the queue's movement. The goal is to analyze the movements of transition zones over a long span of freeway in order to examine any changes in structure. These changes along homogeneous sections as well as inhomogeneous sections such as merges and diverges are analyzed. Moreover, this study seeks to understand the relationship between the characteristics of transition and various traffic and geometric variables.

\subsection{METHODOLOGY OVERVIEW}

Data for this research are observations from inductive loop detectors and vehicle trajectories at various U.S. and non-U.S. freeway locations. All the analyses are done for individual lanes as well as all the lanes combined to examine the differences in features across lanes. Moreover, multiple freeway locations are analyzed to verify reproducibility and compare differences across sites.

\subsubsection{Transition near a Merge}

The transition at a merge is stationary, such that it does not propagate over space as a shock wave. For this type of transition, the length of the transition zone is analyzed using a dataset from the Federal Highway Administration's (FHWA) Next Generation Simulation program (NGSIM, http://ngsim.fhwa.gov). The data consist of individual vehicle trajectories whose resolution is suitable for analyzing this type of transition. Flow, density and speed are employed to examine how their relationships evolve near the inhomogeneous sections. To be specific, a freeway stretch around a merge is divided into multiple contiguous segments, and flow, density and speed are estimated for each segment at 10-second sampling intervals. The results show that the spatial transition in flow takes place in a relatively short distance ( $\sim 90$ meters downstream of the on-ramp) while the transitions in density and space-mean speed occur over longer distances (120 meters and 180 meters on average, respectively).

\subsubsection{Transition near the Tail of a Queue}

The transition zone near the tail-end of a queue moves upstream as the queue grows and downstream as the queue recedes with decreasing demand. The dynamics of the transition zone are studied by analyzing the relationship between transition durations and rates (at fixed locations) and various variables. In this study, a transition rate is defined as the rate of speed change during transition. Detector data from several freeway sites in the U.S. and Europe are selected for this study. The movement and the characteristics of a queue's tail can be traced using the loop detector data that consist of vehicle counts, time-mean speeds and occupancies sampled at 20- to 60-second intervals. Using these data, the beginning and end times (and hence the durations) of a transition at each detector location are measured from 5-minute moving average speeds. Using these measurements, the propagation speed of a queue's tail, as well as the 
changes in transition duration in the direction of the queue's movement, are estimated. Other traffic variables, such as the speeds before and after a regime change, are measured to examine their effect on the transition durations and rates. The results show that transition durations and rates are lane-specific for the onset of queue and are affected by the speed of a shock wave, change in speed and the presence of a merge and/or diverge.

\subsection{ORGANIZATION OF THE REPORT}

The remainder of this thesis is organized into five chapters. Chapter 2 describes the previous research and motivations of the present study. The data processing methodology, analysis and findings of transition characteristics near a merge are described in Chapter 3. Chapter 4 includes the analyses of transitions along shock waves that signal the onset and dissipation of congestion. Finally, a discussion of the results and their implications in relation to the existing macroscopic theory are provided in Chapter 5. Additional tables, figures and plots are included in the Appendix section. 


\subsection{LITERATURE REVIEW}

This chapter provides an overview of the evolution of traffic flow theory over the past half century. Then, the continuum approach and its applications to traffic flow are reviewed. In particular, elaborative discussions of the LWR theory, its numerical solution, empirical support, first-order extensions, and the need for higher-order models are provided. Finally, previous empirical research on the features of transition and the motivations of the present study are presented.

\subsection{HISTORY OF TRAFFIC FLOW THEORY}

The scientific study of traffic engineering originated as early as the 1950s. Traffic-related problems emerged with highway expansions and immense growth in automobile use and, in response, researchers from diverse disciplines including mathematics and physics contributed to the early developments of traffic flow theory.

Wardrop (1952) was among the first to study this evolving discipline. He developed two principles of equilibrium for route choice, now called "Wardrop's Principles," using statistical and mathematical concepts for describing traffic flows. During this decade, the above study was followed by the fluid-dynamic models of traffic flow theory by Pipes (1953), Lighthill and Whitham (1955), and Richards (1956), also known as the LWR Model. These are macroscopic models that describe the average behavior of traffic flow. Newell (1955) came up with a theory about low-density traffic in the same period. Traffic flow theory took a new leap when General Motors' research laboratory started developing car-following experiments and theories (Chandler et al. 1958, Herman et al. 1959, 1961) in efforts to describe traffic at the microscopic level.

Simultaneously, Beckmann et al. (1956) published an economic theory for transportation by studying transportation models, which extended Wardrop's study. This theory introduced the concept of traffic network equilibrium and also laid the foundation for system optimization models in order to reduce cost of increasing flows in congested traffic.

Queueing theory began to emerge by the end of this decade, and was initiated by the work of Mathewson, Trautman and Gerlough (1955) who used the concept of simulation to study traffic flow. They developed models that addressed traffic streams at signalized streets and freeways in terms of delays, arrival and departure times, and turning wait times by using computer programs. Edie and Foote (1958) then studied the kinematics of traffic flow in tunnels and derived various relationships between traffic characteristics. Further approaches in queueing theory were proposed by Carll and Homburger (1962), Cleveland and Capelle (1964), and Newell (1965).

The active research in the 1950s and 1960s nearly discontinued for the next two decades as the key theorists returned to their original disciplines. However, in the early 1990s, researchers found a revived interest in traffic flow. 


\subsection{TRAFFIC FLOW MODELS}

The current section deals with the traffic flow models that describe the movements and interactions among vehicles, drivers and infrastructure. Scientists' approach for discriminating between the traffic flow phenomena is based on three scales of observation in physics: microscopic scale, macroscopic scale and mesoscopic (kinetic) scale.

\subsubsection{Microscopic Flow Models}

The microscopic flow model describes traffic flow based on the detailed interactions between drivers of individual vehicles within a traffic stream. Microscopic properties such as velocity, length, and position of a single vehicle are used to model flow. The car-following models (a specific class of microscopic flow models) are classified based on the approach and logic utilized.

Pipes (1953) introduced the first class of car-following models, the safety distance model. Pipes' rule states: "A good rule for following another vehicle at a safe distance is to allow yourself at least the length of a car between you and the vehicle ahead for every ten miles of hour speed at which you are traveling. " Kometani and Sasaki (1959) assumed that the follower selects a safe following distance based on basic Newtonian equations of motion to avoid a collision. Gipps (1981) proposed an enhancement to this theory by including additional safety reaction time. This model is based on the assumption that the follower should always be able to react to the leader's actions to avoid collisions.

The second class of car-following models is the stimulus-response model, also known as the Gazis-Herman-Rothery (GHR) models which originated from the car-following model proposed by Chandler et al. (1958). The GHR model is regarded as the generalized car-following model, which can be applied to any particular study by modifying the sensitivity term. It is based on the assertion that the follower's acceleration or deceleration (response) is proportional to the relative velocity (stimuli) between the leader and follower. Though a number of extensions for calibrating the parameters have been proposed, the hysteresis phenomenon, spacing criteria, and the acceleration-deceleration asymmetry, made the GHR models contradictory. This resulted in the use of a psycho-physical model, or the Access Point model.

Access Point models describe most of the features of traffic interaction by incorporating real driving behavior. The concept of this model, first introduced by Michaels (1963), states that drivers can detect a vehicle in front of them based on the perceived relative motion, changes in visual angle and change in vehicle size until a certain threshold is reached. After reaching that threshold, the driver decelerates until the relative motion is no longer perceived, but does not reexceed the threshold and continues to act accordingly based on the changes in spacing (Brackstone and McDonald 2000). At smaller spacings and smaller relative motion, this spacingbased threshold is termed as an Action Point. Further study on the threshold values of AP models include the works by Lee and Jones (1967), Evans and Rothery (1977), Leutzbach and Wiedemann, (1986), and, more recently, Reiter (1994) and Kumamoto et al. (1995). In spite of its close resemblance to real-world driver behavior, difficulties in calibration of model parameters and thresholds make the Access Point model less feasible. 
More recent developments in the classical car-following theory include the "intelligent driver model" (IDM) by Trebier and Helbing $(1999,2000,2001)$ and the "human driver model" (HDM) by Trebier et al. (2005a).

An extension in this area was contributed by Newell (2002), who proposed a simplified carfollowing theory based on vehicle trajectories. Newell proposed a simple model for homogenous freeways according to which "a following vehicle has essentially the same trajectory as that of its leader, except for a translation in space and time." This model conforms to the LWR theory with a triangular fundamental diagram (flow-density relation). The simplicity of this model - its less elaborate procedure, fewer parameters and stability - makes it popular in recent times.

Traffic Cellular Automata (CA) modeling is an efficient and speedy approach used in the field of traffic flow modeling for simulating complex networks. It is a simple and discrete microscopic representation of traffic flow in space and time (or velocity). The roadway is divided into cells of constant length (typically 7.5 meters wide with every second corresponding to velocity increments of $27 \mathrm{~km} / \mathrm{hr}$ ) such that each cell is occupied by one vehicle or none. Although the origins of the CA models can be traced back in the 1950s (Neumann 1948), its application to traffic flow was initiated in the late 1990s. The CA models have evolved rapidly in the past decade with the contributions of Helbing (2001), Wolf (1999), Nagel (1996, 2005), Knospe et al. (2004), Nagel and Schadschneider (1992), Schadschneider (2000, 2001, 2002) and many more.

Although there has been extensive research in the car-following theory over the past half century, many contradictions and limitations brought its applicability into question. The validation and calibration methods of these models are questionable due to a large number of parameters to be calibrated and behavioral parameters that are not directly observable in the field. Moreover, lack of information about other factors such as driver, traffic, road and environmental characteristics and their relationships makes the car-following behavior deficient and biased.

\subsubsection{Macroscopic Flow Models}

The macroscopic flow models describe traffic streams at an aggregated level in analogy with fluid dynamics. Density, flow, occupancy and space-mean speed are the four basic macroscopic traffic flow characteristics. The above parameters are defined as follows:

Density: The total number of vehicles in a given section of road (concentration), expressed as vehicles per kilometer.

Flow: The total number of vehicles passing a certain point in a given time, expressed as vehicles per hour.

Occupancy: The percentage/fraction of time the detector is occupied by a vehicle.

Space-Mean Speed: The total distance traveled by all of the vehicles on a given length of a road, by the total time spent in the region, measured in kilometers per hour. 
Macroscopic traffic flow models are used to establish relationships among the macroscopic traffic variables. The fluid dynamics analogy treats traffic stream as a one-dimensional compressible fluid which results in two common assumptions: continuum hypothesis and flow conservation.

\subsubsection{The Continuum Hypothesis - Conservation Law}

The continuity hypothesis associates traffic with fluid flow, thus considering traffic stream as continuous media by assuming a continuous distribution of all variables (densities, flows and speeds) at each point in space and time. Navier (1822) and Stokes (1845) shed light on the fluid-dynamic approach (i.e., the continuum approach) by formulating a set of non-linear partial differential equations (PDEs) also known as the Navier-Stokes Equations (NSE) which describe the motion of a fluid. The most popular application of NSE is the conservation law, which states that "net flux is accompanied by an increase or decrease of material (fluid)" (Maerivoet and Moor 2005). Depending on whether the fluid is compressible or incompressible, the appropriate approach for solving NSE is selected. Traffic is generally considered an inviscid compressible fluid.

The conservation/continuity equation as applied to define traffic flow states that "the rate of change of flow and density in space and time are consistent with no entering/leaving traffic" (Daganzo 1997b), by considering a continuous media and allowing discrete increments to be infinitesimal. The equation is given by:

$$
\frac{\partial k(x, t)}{\partial t}+\frac{\partial q(x, t)}{\partial x}=0
$$

where, $k$ and $q$ are, respectively, the density (number of vehicles per unit distance) and flow (number of vehicles passing a given point in unit time) at the space-time point $(x, t)$ on a roadway. The above equation (1), which is a first-order PDE, was later used to develop a number of traffic flow theories and to describe the fundamental relationship between traffic parameters.

\subsubsection{Lighthill-Whitham-Richards Theory: Kinematic Wave Theory}

Lighthill and Whitham (1955) developed a traffic flow theory using the first-order continuum model of traffic flow. The fundamental hypothesis of this theory is that "at any point of the road, flow is a function of concentration," which gave rise to the flowconcentration curve (fundamental diagram). Transitions or disruptions in flow propagate in space as kinematic shock waves whose velocities are given by the slopes of the curve. The theory is applicable to all traffic concentrations and also to flow near junctions. Richards (1956) developed a similar theory of traffic flow by deriving an empirical relation between density and speed to study the formation of shock waves. Since this theory was developed by the three authors during the same period with similar solutions to the traffic problems, it is more popularly known as the Lighthill-Whitham-Richard (LWR) model. 
A number of formulations were applied to solve the LWR model using the above hypothesis. LWR states that flow rate is a function of density, implying:

$$
q=q(k)
$$

The conservation law of traffic flow can now be expressed as:

$$
\frac{\partial k(x, t)}{\partial t}+\frac{\partial q(k(x, t))}{\partial x}=0
$$

The velocity of the shock waves is formulated as follows:

$$
v_{s}=\frac{q\left(k_{2}\right)-q\left(k_{1}\right)}{k_{2}-k_{1}}
$$

where,

$k_{1}, k_{2}:$ densities upstream and downstream of the shock wave;

$q\left(k_{1}\right), q\left(k_{1}\right)$ : corresponding solutions to 2 .

The LWR theory forms the basis for many traffic flow theories because of its simplicity and ability to clearly represent traffic flow features. This theory extended the scope and validity of the flow-concentration curve by including non-stationary traffic conditions. The major advantage of the elegant LWR model over other models is its easy implementation to various traffic phenomena with low computational complexity. Traffic researchers came up with numerous solutions to the LWR model over the past few decades. Newell proposed a simplified kinematic theory (Newell 1993) using cumulative count curves instead of shock waves as a solution to the LWR theory. An overview of the empirical studies supporting the LWR theory is also provided in the following sections.

\subsubsection{Newell's Simplified Kinematic Wave Theory}

In an attempt to simplify the evaluation of traffic characteristics, (flow-density), Newell (1993) proposed a simplified kinematic wave theory that expressed the conservation law using cumulative flow. Cumulative curves were used rather than following the path of shock waves to analyze traffic dynamics, particularly queuing behind a bottleneck. This theory enabled approximation of differential equations of fluid-dynamic theory with negligible loss of accuracy, and it also avoids mathematical nuisances with the use of triangular fundamental diagram. Another favorable feature is its ability to predict traffic variation at end points without evaluating traffic behavior at intermediate points (multidimensional flows - entrance and exit ramps). The simplified theory also provides a graphical representation of the LWR model for both free-flow and congested conditions with the use of piece-wise linear flow-density relations for analyzing traffic states.

Figure 2 depicts the triangular model of Newell, where the points on the diagram describe possible traffic conditions (or "steady states"). For every flow, there are two densities 
(congested and uncongested). The main advantage of the triangular model is its ability to predict traffic features that are not described by the non-linear flow-density relations. Also, this model is used extensively due to its simple piece-wise linear flow-density relations and better approximation ability without much loss of accuracy.

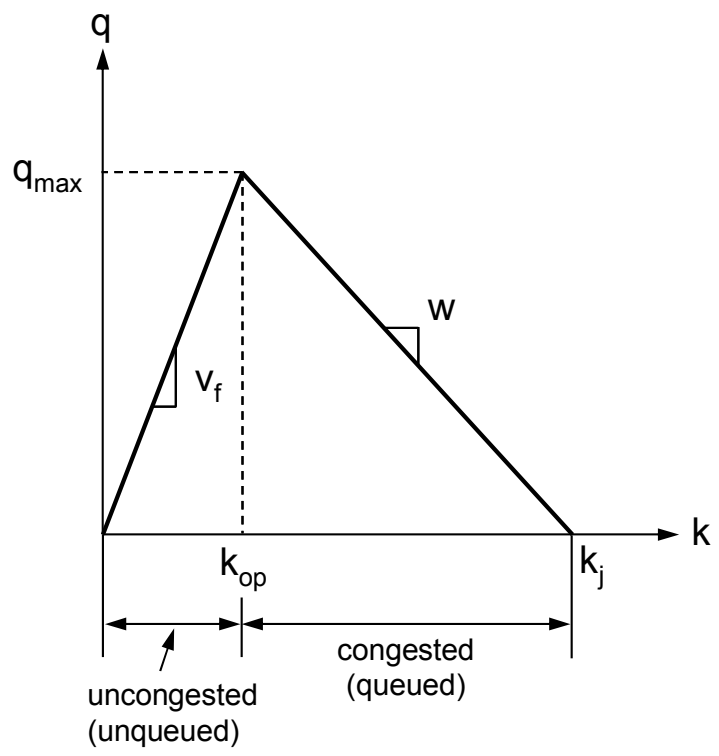

Figure 2.1: Triangular fundamental diagram

The corresponding flow equations governing this model are given below:

$$
\begin{aligned}
q(k) & =\min \left(v_{f} k, \quad w\left(k-k_{o p}\right)+q_{\max }\right) \\
& =\min \left(v_{f} k,-\frac{v_{f} k_{o p}}{k_{j}-k_{o p}}\left(k-k_{o p}\right)+v_{f} k_{o p}\right) \\
q_{\max } & =v_{f} k_{o p} \\
w & =-\frac{v_{f} k_{o p}}{k_{j}-k_{o p}}
\end{aligned}
$$

where,

$q_{\max }$ is the maximum flow

$v_{f} \quad$ is the mean free flow speed

$k_{o p} \quad$ is the optimal density

$k_{j} \quad$ is the jam density (where flow is almost zero)

$w \quad$ is the velocity (negative) moving against the flow during congestion.

The techniques developed by Newell have become the standard tools for analysis of freeway traffic, especially queue formation and propagation, bottleneck analysis and intersection capacities evaluation. 


\subsubsection{Empirical Support of First-Order Model}

The seminal works of traffic flow theory and traffic flow modeling over the past 50 years give us a better understanding of complex traffic phenomena. When applied to traffic data, these flow models should reflect real traffic characteristics and real driver behavior. In order to verify the reality of such theories, empirical investigation of traffic features is conducted. An overview of some recent empirical studies that demonstrate the usefulness of the LWR model is presented in this section.

Cassidy (1998) quantified the bivariate relations between traffic variables under nearly stationary conditions by investigating cumulative curves of vehicle counts and occupancies. Near-stationary periods were identified, and scatter-plots of congested flowoccupancy were produced using aggregated data over the stationary periods. These resulting plots revealed the existence of a relationship between queued traffic flow and occupancy, thus conforming to the kinematic wave theory.

Cassidy and Mauch (2001) did some further empirical investigation to observe freeway oscillations. Oscillations were found to form and grow near freeway interchanges and displayed regular features that did not affect the queued traffic characteristics. The study confirmed that in moderately dense queues, the formation of oscillations is more closely related to lane-changing phenomenon than to the car-following mechanism. The propagation of oscillations was well explained by the LWR theory with minute errors.

Although the empirical studies revealed anticipated results in accordance to the firstorder traffic flow model, its inability to represent certain traffic features (mainly driver differences, shock wave propagations and traffic instabilities) makes it less feasible to report certain traffic phenomena. Some of the major traffic features unaddressed by the LWR model include traffic oscillations, hysteresis phenomenon, instantaneous transitions, capacity drop, etc. There are two ways to address these deficiencies - (i) resorting to advanced extensions of the first-order models or (ii) using higher order traffic flow models.

\subsubsection{Extensions to the First-Order Model}

Numerous extensions to the first-order model have been proposed by various researchers such as Newell (1993), Lebacque (1996, 2002), Nelson and Hu (2000), and Cassidy and Chung (2002) to account for the deficiencies mentioned in the previous section. Daganzo, in association with other researchers, developed several extensions to the first-order traffic flow theory, dealing with vehicle interactions, congestion near diverges, lanechanging phenomenon and moving bottlenecks.

Daganzo (1997a), who studied traffic dynamics on freeway sections with special lanes and two vehicle types, developed a generalized theory of kinematic waves. The theory deals with queued conditions with respect to the two vehicle classes resulting in 2-pipe regime and 1-pipe regime effects that are not recognized by the classic LWR theory. A solution to this problem was presented in an extension of this study, which used a simple physical principle that is equivalent to the Godunov's method for simulation of 
homogenous freeways (Daganzo 1997c). This principle also was used in describing congestion near freeway diverges.

As an extension, Daganzo (2002a) presented a traffic dynamics theory for homogenous, multilane freeway flow by considering two classes of drivers - aggressive drivers (rabbits) and timid drivers (slugs). By incorporating human behavior, this theory was able to predict the capacity drop and hysteresis phenomenon. Further, the merging phenomenon and queue formation process were described by the above behavioral theory by assuming a fixed buffer zone downstream of the on-ramp. Traffic behavior at the entrance and merge, and the onset of congestion at the merge, were explained with the help of a pumping mechanism. According to this mechanism, shorter headways are observed downstream of an on-ramp, resulting in a capacity flow regime (Daganzo 2002b).

Munoz and Daganzo (2003) presented a complete picture of moving bottlenecks by using the behavioral theory of rabbits and slugs. Their empirical studies contradicted existing theories, which resulted in a new theory that is consistent with the experimental data. According to this new theory, a moving bottleneck is treated as a boundary condition by integrating with the LWR theory, thus accounting for driver differences and vehicle types. Though this theory performed well, it did not yield consistent results when applied to light traffic with high-speed bottlenecks.

In the context of moving bottlenecks, Daganzo and Laval (2005) developed a numerical scheme by treating moving bottlenecks as a sequence of fixed obstructions at locations with the same capacity. This theory can be used to model bottlenecks with endogenous and exogenous trajectories, multiple bottlenecks, and traffic streams with buses/trucks by considering suitable car-following laws and interaction rules. The numerical treatment allows for a more accurate prediction of average flows, densities, accumulation and delays. In 2006, they proposed a theory to capture lane-changing effects by using multilane models of moving bottlenecks. The theory suggested that lane-changing phenomenon results in voids in traffic streams that reduce flow. Effects such as a drop in discharge rate and the relationship between bottleneck speed and capacity also were studied without any recalibration.

Though the extensions of the first-order models discussed above address most of the LWR theory's shortcomings, certain drawbacks still exist. The LWR model lacks accuracy since it assumes instantaneous flow (or speed) and neglects the dynamic flow phenomena. Since all the extensions rely on the LWR theory, the validity of these theories is questionable when applied to light traffic with high speeds. Also, the reason behind the strange effects predicted by the underlying assumptions of these theories, especially for moving bottlenecks, remains unanswered. In order to account for lanechanging phenomenon, artificial constants are employed, thus questioning the validity and calibration of these models. The following section gives an overview of higher-order models that have been developed to overcome the deficiencies of first-order LWR models and their extensions. 


\subsubsection{Higher-Order Models}

Payne $(1971,1978)$ and Whitham (1974) were among the first to introduce the concept of higher-order models by including driver reaction time and considering momentum equations in car-following models. The major drawback of this model is its unsatisfactory performance with respect to congestion, lane drop, stop-and-go waves, and on-ramp inflow. These shortcomings were addressed by certain extensions proposed by Cremer and May (1985) and Papageorgiou et al. (1990). Zhang (1998) proposed an extension to the momentum equations, similar to Payne's theory which satisfied the stability condition with respect to shock waves. The major improvement in the higher-order models when compared to the first-order models is the inclusion of dynamic characteristics, which incorporate transitions between equilibrium and non-equilibrium states. However, this is achieved at the expense of violation of the anisotropic property (Zhang 2001). Further progress in the higher-order models includes work by Kuhne (1984), Castillo and Benitez (1995), Helbing (2001) and Zhang (2003).

Although higher-order models provide solutions to the shortcomings of the first-order models, these theories face a number of criticisms due to the physical and logical flaws involved in deriving the models. Daganzo (1995) provided an overview on the flaws of higher-order models. The PW model developed by Payne (1971) and Whitham (1974) using approximations to the car-following differential equations received criticisms due to its logical flaws. Daganzo questioned the methodology behind neglecting higher-order derivatives of spacing and speeds in the PW model. He also provided an illustration of the complicated PDEs used in higher-order models, which predicted negative flows and negative speeds. In addition, higher-order models do not have numerical solution schemes, thus making these models difficult to implement for larger networks.

Despite their contrasting methodologies and approaches, the extensions to the first-order model and higher-order models are both equally popular in resolving the LWR model's shortcomings. Each approach has its own advantages and disadvantages. In conclusion, it can be noted that the simplicity and elegance of the first-order model make it more suitable for capturing traffic flow characteristics, while the higher-order models are best suited for studying higher-order effects such as shock waves, diffusion, etc (Zhang 2001).

More details on the empirical studies of traffic transitions and the motivations behind the present research are provided in the following section.

\subsection{EMPIRICAL STUDIES OF TRANSITION ZONE}

Muñoz and Daganzo (2003) studied "transition zones" that emerge when a queue forms at a bottleneck, propagates as a "shock" upstream and then dissipates with decreasing demand. This study was conducted on a California freeway over two loop detector locations on a weekday. Using the loop detector data, they found that the transition occurred as predicted by the LWR model: data points on the flow-occupancy curve moved from the uncongested to congested branches (and vice versa), and the transition zone propagated upstream at the speed predicted by the kinematic wave model. Moreover, they estimated the error by assuming instantaneous 
transition based on the estimated transition duration (several minutes) and the speed of a shock wave. The resulting error was within five-vehicle spacing, indicating that the accuracy of the LWR model suffers little from assuming instantaneous transitions. However, the shocks and transition zones were traced over a short distance (two loop detector locations) only on a single day. Thus, factors that influence the transition process could not be studied.

The LWR theory describes traffic states and their dynamics within a homogeneous section where flow is conserved. Thus, applying this theory becomes cumbersome when traffic flow is not conserved due to merging and diverging flows at freeway interchanges. Newell (1993) addressed this shortcoming. He treated the merges and diverges as points where discontinuities in traffic state occur (instantaneously). Then, a traffic state downstream of the merge exhibits a flow that is larger by the inflow than the state upstream of the merge. This implies that in a queued state, vehicles accelerate instantaneously at the merge and display trajectories that are piece-wise linear. A similar logic applies to a diverging case such that a diverging point defines the boundary between downstream and upstream states, with a larger flow upstream of the merge. In reality, merging and diverging take place over some time and distance due to systematic lane changes (to reach destination or exit lanes) and finite acceleration and deceleration capabilities.

The present study investigates the transition features that occur by merging flow as well as regime changes at the onset and recovery of queues. The aim is to study transition zones at the tails of queues over long segments over multiple days in order to obtain statistically significant results. Other variables that affect the characteristics of transition zones also are analyzed. 


\subsection{TRANSITIONS NEAR A MERGE}

\subsection{INTRODUCTION}

An investigation of the literature shows that inhomogeneous features on freeways, such as freeway ramps, lane reductions and grade changes, tend to change the fundamental relationships among traffic parameters. This section illustrates the spatial characteristics of transitions near a merge during congestion and its relationship with traffic variables (e.g., inflow, change in speed and density, etc.) by using trajectory data from different sites. The primary objective here is to identify the distance over which transition occurs near a freeway merge. The spatial evolution of the fundamental relationships between macroscopic traffic parameters such as flow and density also is studied.

\subsection{STUDY SITE AND DATA DESCRIPTION}

A merge is analyzed using the trajectory data from eastbound Interstate 80, (I-80) near San Francisco, CA, as shown in Figures 3.1(a) - c). This site is ideal for studying transitions near a merge as it contains an on-ramp and a relatively long, homogeneous section downstream of the merge. The data for this site are publicly available through the FHWA's Next Generation Simulation program (NGSIM, http://ngsim.fhwa.gov/).

The study area is approximately 500 meters long, with an on-ramp at Powell Street and an offramp at Ashby Avenue downstream of the study area. There are six lanes that are numbered from 1 to 6 , starting from the farthest left lane. The farthest left lane (lane 1) is the high-occupancy vehicle (HOV) lane. The Powell Street on-ramp is located about 150 meters downstream of the study area's upstream boundary, as shown in Figure 3.1(a). Both vehicle trajectory data and detector data are used for the study. The details of the data collection method and some preliminary analyses are provided in Hranac et al. (2004).

\subsubsection{Trajectory Data}

Vehicle trajectory data are transcribed from the video data collected using seven video cameras, as shown in the aerial picture in Figure 3.1(b). The videos were collected on April 13, 2005 during the afternoon rush and consist of three separate 15-minute periods: 1) 4-4:15 p.m.; 2) 55:15 p.m.; and 3) 5:15-5:30 p.m. The data for the 4-4:15 p.m. period primarily represent transitional traffic conditions during the build-up of congestion. The remaining two periods represent congested traffic conditions. These data provide latitudinal and longitudinal coordinates of each vehicle and other parameters, such as velocity, acceleration, spacing, headway and vehicle type, every 1/10th of a second. 


\subsubsection{Detector Data}

Inductive loop detector data are collected through the Berkeley Highway Laboratory, (BHL) between Powell Street and Gilman Avenue on I-80 in Emeryville, CA. Data collected at station 7 on April 13 is used to complement the vehicle trajectory data by analyzing the vehicle count, speed and occupancy data. Of note, station 7 is the closest location from which the trajectory data were collected.

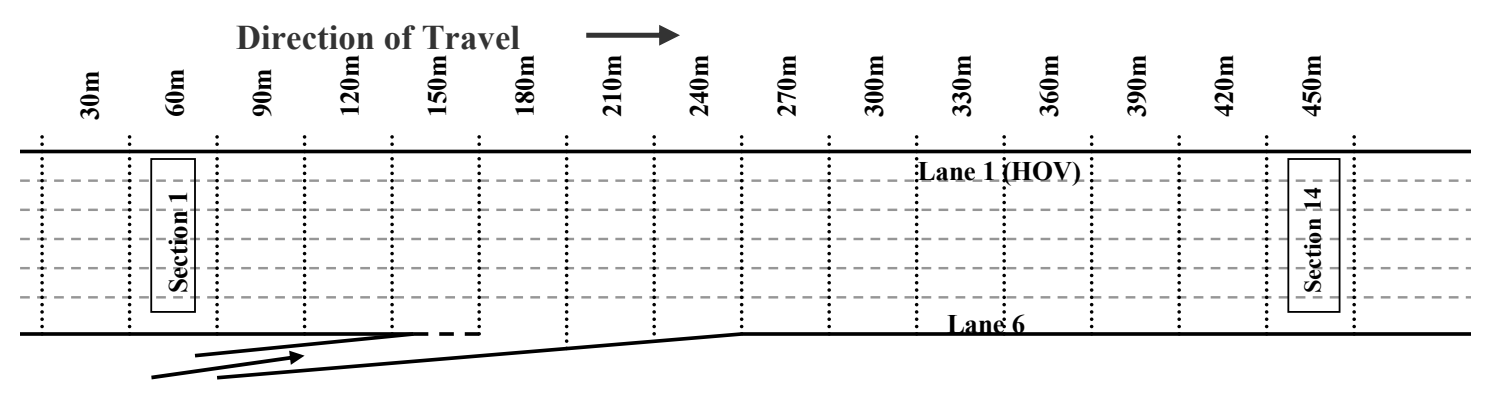

From Powell St

(a)

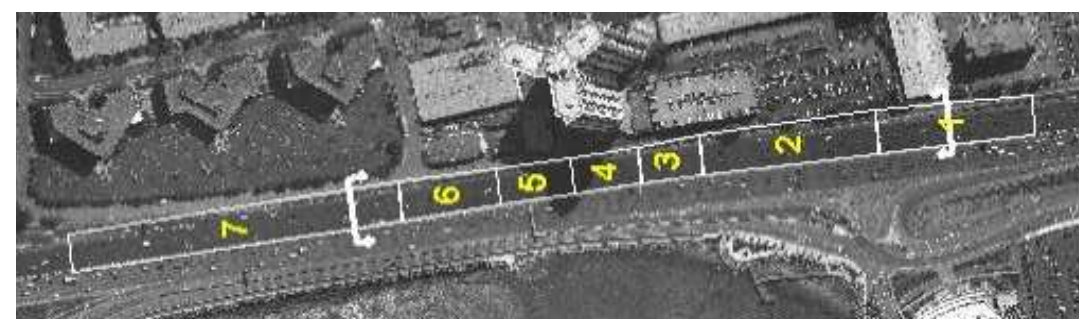

(b)

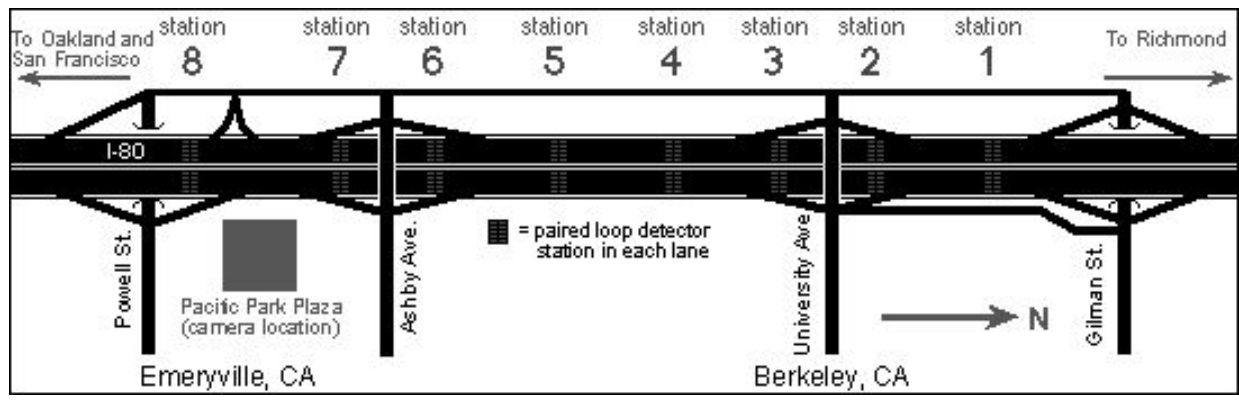

(c)

Figure 3.1: Eastbound Interstate 80 Study Area [Adopted from http://ww.ngsim.fhwa.dot.gov]

(a) site schematic; (b) aerial photo; (c) detector locations 


\subsection{METHODOLOGY}

The main objective is to study the nature and effect of the transitions that occur near a freeway merge in a given section. In order to achieve this, the evolution of macroscopic traffic variables, such as flow, density and space-mean speed, is traced along kinematic waves. The methodology for the analyses includes the following steps:

1. Divide the freeway study section into contiguous segments of 30 meters. Thus, the study section is divided into 14 subsections, as shown in Figure 3.1(a).

2. Measure the headway and spacing every 10 seconds in each subsection.

3. Compute the average values of macroscopic traffic variables (flow, density and spacemean speed) for every 30 meter x 10 second time-space region.

4. Identify near-stationary time-space regions from speed contours and rescaled cumulative curves of speed (or flow).

5. Identify the distance over which a transition zone occurs near the merge in terms of flow, density and speed changes.

\subsubsection{Data Processing}

Trajectories are curves in the time-space diagram that define the position of a vehicle for every moment of time $x(t)$. In the present study, I-80 trajectory data, as exemplified in Figure 3.2, is used to comprehend the nature of transition due to the merge (on-ramp) at Powell Street. Since it is difficult to measure the length of this transition and its effect on macroscopic traffic variables directly from the trajectories, the data are processed in order to achieve the necessary resolution for the analysis. To this end, the freeway study section is divided into multiple contiguous segments of 30 meters. Thus, the entire length of 500 meters is divided into 14 subsections, the first one being the section from 30 meters to 60 meters and the last section 420 meters to 450 meters (see Figure 3.2).

Traffic flow characteristics in each subsection are estimated by measuring the average spacing and headway values at 10 -second intervals from the original trajectory data, where headway is a time separation between vehicles and spacing is a distance between vehicles. Thus, average headway $\left(h_{i t}\right)$ and average spacing $\left(s_{i t}\right)$ are calculated in each time-space region (subsection) shown in Figure 3.1(a) on an individual lane basis, where $i$ denotes the section number and $t$ denotes the time interval. Then, by definition, flow $\left(q_{i t}\right)$ and density $\left(k_{i t}\right)$ are respectively reciprocals of $h_{i t}$ and $s_{i t}$, and space-mean speed $\left(v_{i t}\right)$ is a ratio of flow to density.

In order to understand the dynamic traffic and flow characteristics near the on-ramp, all of the above parameters are quantified for all lanes combined in order to ensure conservation of flow. Thus, the total flow and density are taken as the sums of the flows and densities of individual lanes, and the space-mean speed is the average of the speeds of individual lanes weighted by densities. Using these measurements various plots, such as speed contours, time-series flow, density and space-mean speed, and oblique and moving average curves, are then constructed. 


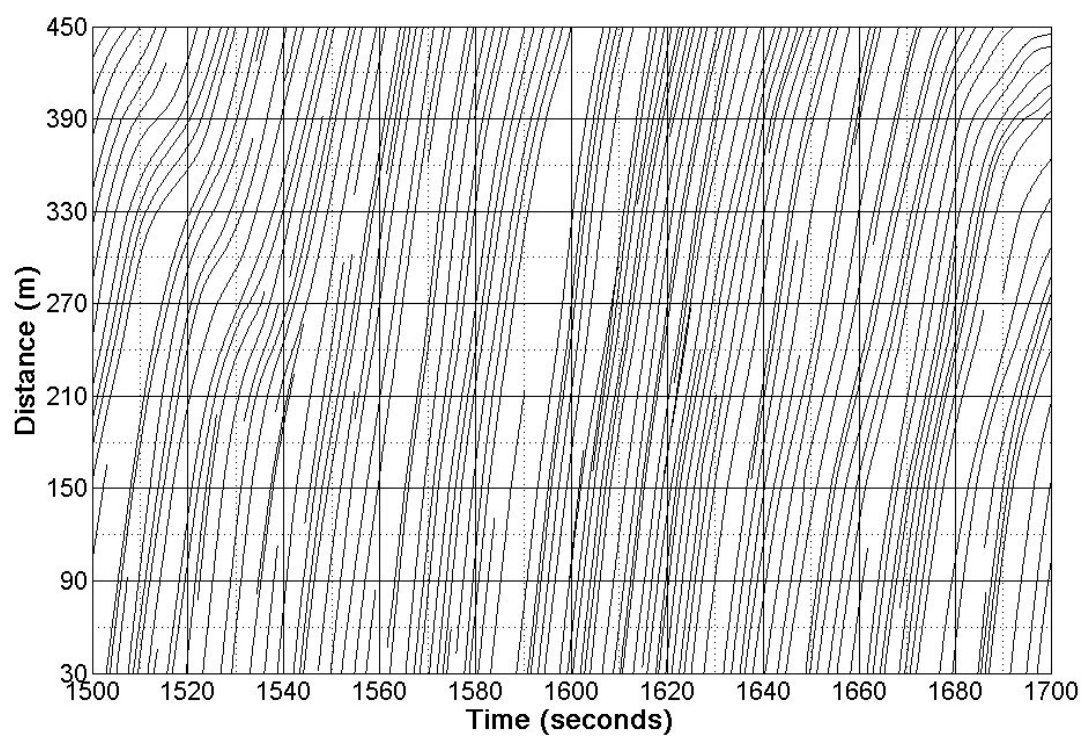

Figure 3.2: Trajectory Data Divided into Segments of 30 Meters Every 10 Seconds

\subsubsection{Preliminary Analysis}

According to the LWR theory, changes in (stationary) traffic states propagate as kinematic waves whose speeds are determined by the fundamental relationship between flow and density at equilibrium. To reduce the noise in the measured relations, near-stationary states are identified as the time-space regions bounded by two neighboring kinematic waves. Then, data corresponding to these periods are extracted to study the evolution of traffic parameters around the merge. To identify these near-stationary time-space regions, speed contour plots and rescaled (oblique) curves of cumulative space-mean speed are used.

Speed contours depict general conditions on the study section over time and space. Speed contour plots, shown in Figure 3.3, are developed for the time periods 4-4:15 p.m.and 5-5:30 p.m. with a resolution of 10 seconds by 30 meters. Time is shown on the x-axis, the study sections are shown on the $y$-axis, and the estimated space-mean speeds are represented using a grey scale according to the legend in the figure (darker shades represent lower speeds and lighter shades represent higher speeds). The plots show that the average speed ranged from near 0 to 55 $\mathrm{km} / \mathrm{hr}$, indicating heavy congestion for this facility. Several kinematic waves signaling a series of decelerated (darker regions) and accelerated states (lighter regions) characterizing stop-and-go traffic can be seen in the plots.

Analyses of these plots indicate that there are four such regions in the 4-4:15 p.m. period and seven such regions in the 5-5:30 p.m. period. These regions are mapped by dotted lines in the figure and also are summarized in Table 3.1. The kinematic waves travel from section 14 to section 1 with an average wave speed (backward moving wave speed) of $17.55 \mathrm{~km} / \mathrm{hr}$ (computed by tracing the arrival times of each wave at the downstream and upstream ends). The nearstationary states identified from the speed contours are further confirmed by the oblique cumulative curves of space-mean speed and flow. 


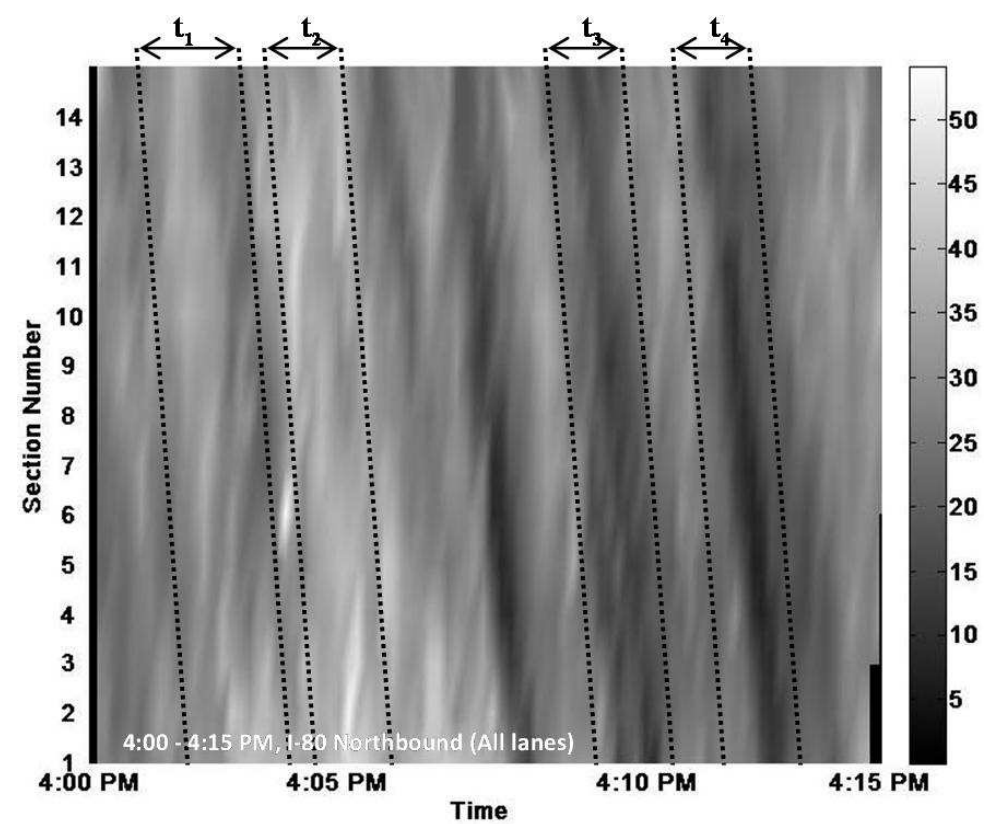

(a)

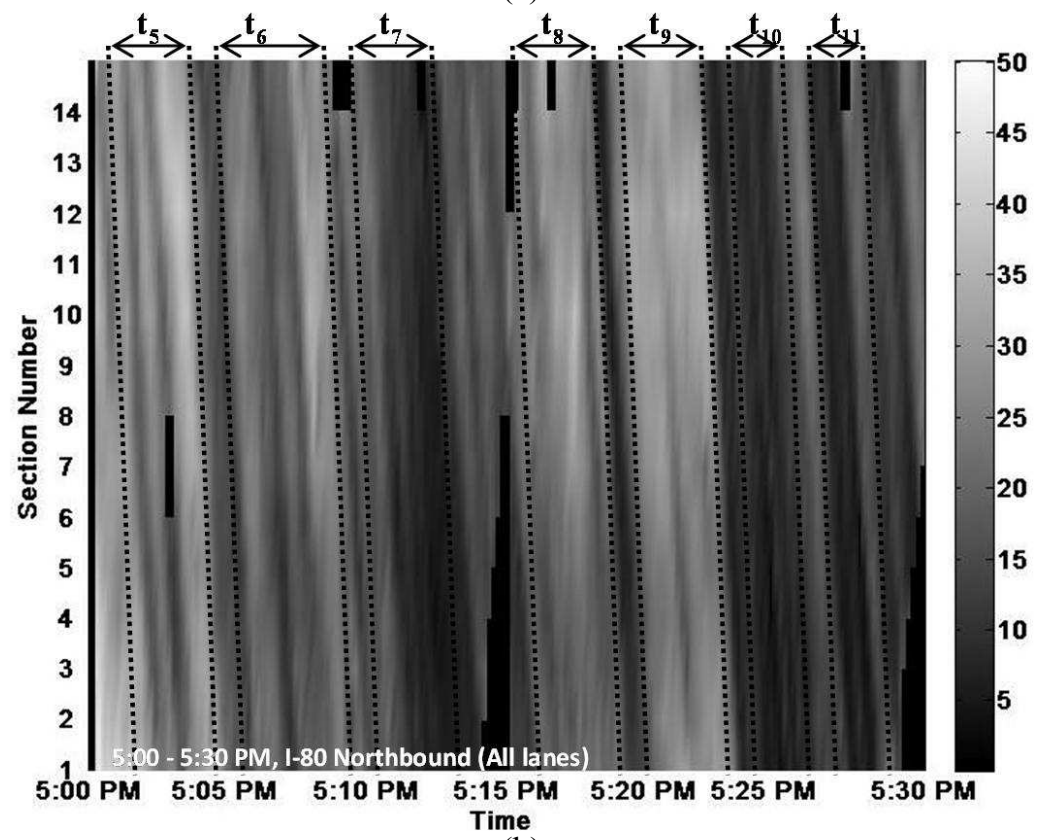

(b)

Figure 3.3: Speed Contours of I-80 Showing Near-Stationary Time-Space Regions $\left(t_{5}\right.$ to $\left.t_{11}\right)$ (a) 4-4:15 p.m. (b) 5-5:30 p.m.

Time-series plots of count, occupancy and speed typically display large noise, making it difficult to identify different traffic states and the times of state changes. To suppress the noise in the data and reveal the trends better, cumulative curves of traffic observations are constructed on an oblique time axis. This technique is described in detail in numerous papers, such as Munoz and Daganzo (2003). In orthogonal time axis, constructing an oblique curve is equivalent to 
constructing a cumulative curve and then rescaling it by subtracting background reductions. More specifically, if $V(x, t)$ gives the cumulative space-mean speed values at section $x$ and time $t$, rescaled values are obtained by subtracting a factor $V_{0}\left(t-t_{0}\right)$ from each value, where $V_{0}$ is a scaling factor and $t_{0}$ is the starting time of the curve. The value of $V_{0}$ is established by an iterative process until the arrival time of each wave (and each time-space region) can be clearly identified. A wave's arrival time is characterized by a change in slope of the rescaled curve. Thus, the nearstationary time-space regions are selected where the slope of the rescaled curve in the interval can be defined by a linear approximation.

Figures 3.4(a) and (b) present the rescaled cumulative curves of space mean speed $V(x, t)-V_{0}(t$ $t_{0}$ ), versus time at section 14 in the time-periods 4-4:15 p.m. and 5-5:30 p.m., respectively. The near-stationary regions are identified as the periods of nearly constant slopes (space-mean speed), which are marked with solid lines and labeled as $t_{1}$ to $t_{11}$ in the figure. The corresponding beginning and ending times of the selected time periods also are marked. For example, the nearstationary interval, $t_{1}$, corresponds to the time period 4:01:10-4:02:40 p.m.

The selected regions, their beginning and ending times at section 14, and their respective wave speeds are summarized in Table 3.1. Similarly, the near-stationary regions are determined for all the upstream sections based on the wave speeds given in Table 3.1 and are further confirmed by examining the slopes of the rescaled curves.

\begin{tabular}{cccc}
\multicolumn{5}{c}{ Table 3.1: Time Periods of Near-Stationary Traffic States at Section $\mathbf{1 4}$} \\
\hline $\begin{array}{c}\text { Region ID } \\
\left(\mathbf{t}_{\mathbf{i}}\right)\end{array}$ & $\begin{array}{c}\text { Start Time } \\
(\boldsymbol{h} \boldsymbol{h}: \mathbf{m m}: \mathbf{s s})\end{array}$ & $\begin{array}{c}\text { End Time } \\
(\boldsymbol{h} \boldsymbol{h}: \mathbf{m m}: \mathbf{s s})\end{array}$ & $\begin{array}{c}\text { Wave Speed } \\
(\mathbf{k m} / \mathbf{h} \boldsymbol{r})\end{array}$ \\
\hline $\mathrm{t}_{1}$ & $4: 01: 10$ & $4: 02: 40$ & 15.60 \\
$\mathrm{t}_{2}$ & $4: 03: 00$ & $4: 04: 30$ & 15.60 \\
$\mathrm{t}_{3}$ & $4: 08: 40$ & $4: 10: 00$ & 20.06 \\
$\mathrm{t}_{4}$ & $4: 11: 20$ & $4: 12: 40$ & 20.06 \\
$\mathrm{t}_{5}$ & $5: 01: 10$ & $5: 03: 50$ & 20.06 \\
$\mathrm{t}_{6}$ & $5: 04: 00$ & $5: 08: 50$ & 12.76 \\
$\mathrm{t}_{7}$ & $5: 09: 50$ & $5: 12: 40$ & 15.60 \\
$\mathrm{t}_{8}$ & $5: 15: 20$ & $5: 18: 00$ & 20.06 \\
$\mathrm{t}_{9}$ & $5: 19: 00$ & $5: 20: 40$ & 17.55 \\
$\mathrm{t}_{10}$ & $5: 22: 00$ & $5: 24: 30$ & 20.06 \\
$\mathrm{t}_{11}$ & $5: 25: 40$ & $5: 26: 50$ & 15.60 \\
\hline
\end{tabular}




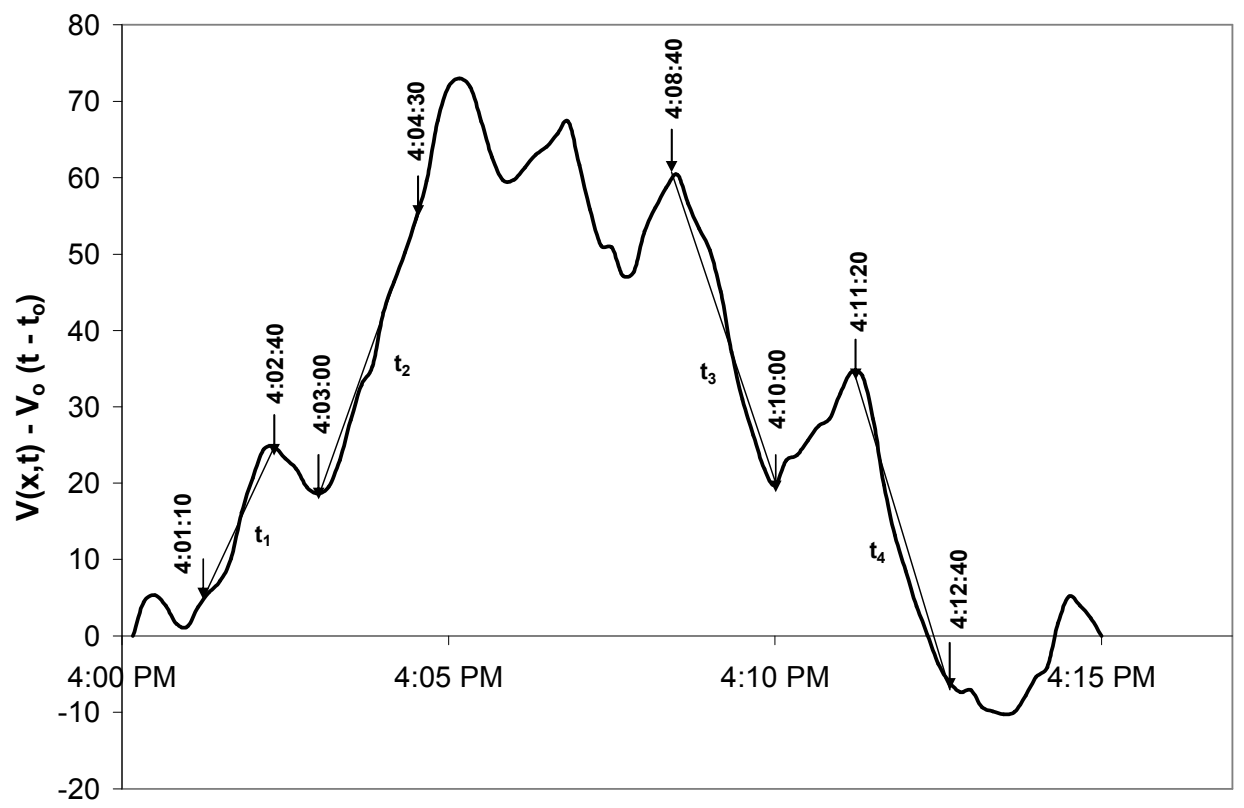

Time, @ section 14

(a)

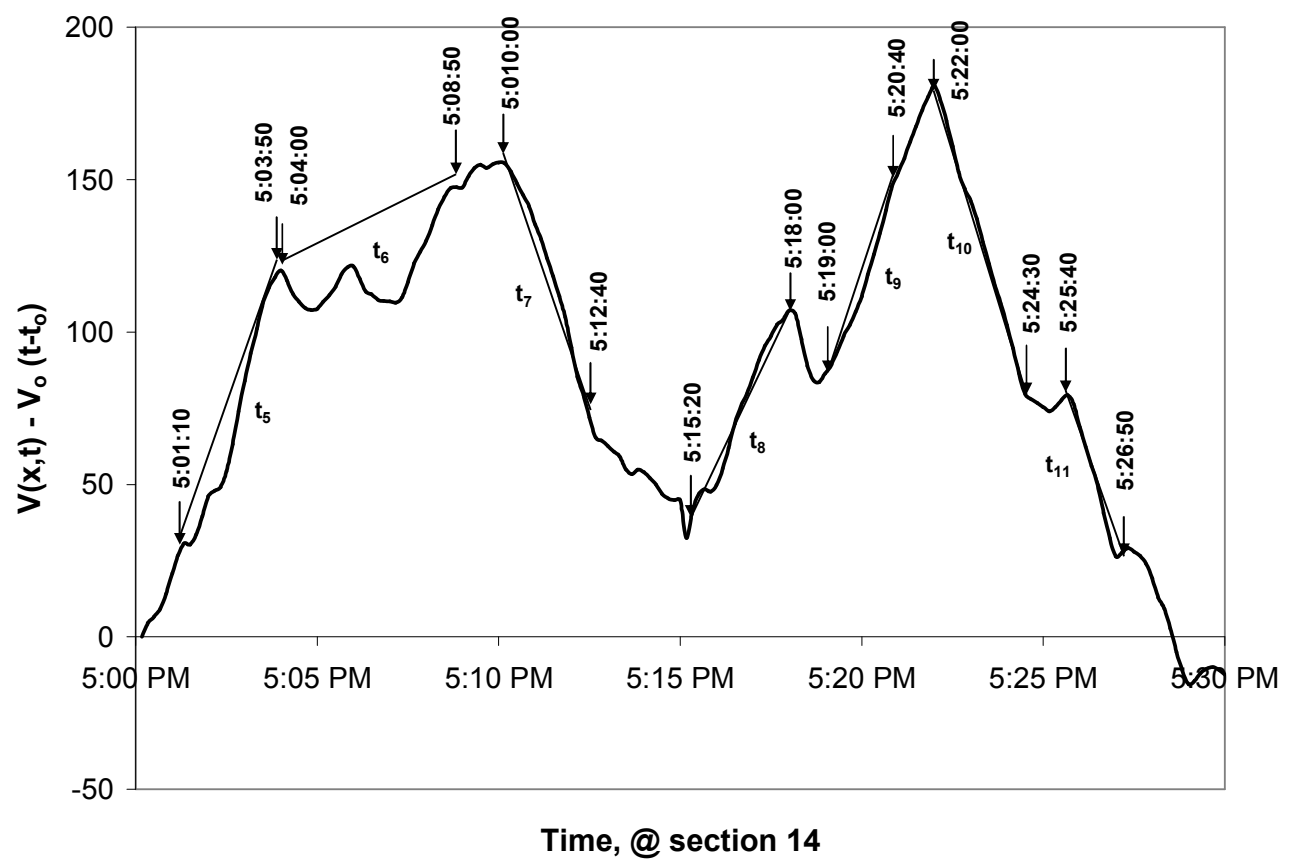

(b)

Figure 3.4: Rescaled Cumulative Curves of Space Mean Speed at Section 14 (a) 4-4:15 p.m. (b) 5-5:30 p.m. 


\subsubsection{Analysis}

The evolution of the traffic parameters across the sections in each time-space region $t_{i}$ is studied to measure the transition length due to the on-ramp inflow. Then, the effect of potential explanatory variables, such as change in flow (inflow), density and/or speed due to merging flow on transition length, are studied. Finally, deviations in the equilibrium state due to transition are investigated from the fundamental diagram of a flow-density curve.

In order to study the spatial evolution of the traffic parameters, averaged values of flow, density and space-mean speed are computed across time in each near-stationary time-space region $\left(t_{i}\right)$ identified. The averaged values obtained for each stationary region are then plotted against the sections (or distance $x$ ). For the 11 regions identified, plots of flow vs. section, density vs. section and space-mean speed vs. section are developed. Figures 3.5(a) - (c) present an example of flow, density and space-mean speed over the 14 segments during a stationary period of 5:185:23 p.m. $\left(t_{9}\right)$. Each plot shows the evolution of flow, density and speed marked by the passage of two neighboring kinematic waves. As noted on the plots, traffic moves from segments 1 to 14, and the waves propagate against traffic flow in congestion. The Powell Street on-ramp is located in section 4, where a discontinuity in state is expected to occur. It also is expected that the sections upstream of the on-ramp exhibit higher density and lower flow and speed as compared to the sections downstream. The length of transition is measured in two parts; upstream and downstream of an on-ramp. The study site was not long enough to measure the length of transition upstream. Thus, this study focuses on the length of transition observed downstream of the on-ramp. The following observations are noted from the plots.

Flow: As shown in Figure 3.5(a), an abrupt increase in flow is observed starting at section 4 and ending at section 8 . After the substantial increase (nearly 1,500 vph), the flow stabilizes and remains nearly constant over the rest of the freeway sections. The length of the transition zone in this case is thus measured as 90 meters. A similar pattern of substantial increase followed by stabilization is observed in the other 10 near-stationary periods. The transition length with respect to flow varied between 60 meters and 120 meters with an average change in flow of 960 vph.

Density: It is observed that the transition in density is gradual and persists over a longer stretch of freeway as shown in Figure 3.5(b). Note that in the near-stationary period $t_{9}$, the density continues to increase until section 4 and then gradually decreases until section 10 . Hence, the transition length downstream of the ramp is about 180 meters with a decrease in density of about $50 \mathrm{veh} / \mathrm{km}$. Another notable feature is that a decrease in density begins at sections further ahead of the on-ramp location. For period $t_{9}$, the density remains high until section 7 and then starts to decrease. A similar pattern is observed for the other 10 stationary periods such that the average change in density is about $46 \mathrm{veh} / \mathrm{km}$, with the transition length ranging from 90 meters to 180 meters.

Space-Mean Speed: Transition in space-mean speed displays a similar pattern as the density. As illustrated in Figure 3.5(c), the speed transition has already begun in section 1 and the speed continues to decrease until section 4. Due to traffic inflow from the on-ramp, speed increases gradually from section 4 and the transition continues until section 12 . Thus, the length of 
transition upstream is at least 90 meters and the length downstream is about 240 meters, with an increase of about $11 \mathrm{~km} / \mathrm{hr}$. The length of the transition (downstream of the ramp) in speed is the largest compared to flow and density and ranges between 120 meters and 240 meters with an average speed increase of $6 \mathrm{~km} / \mathrm{hr}$. A slump in the speed is observed near the downstream end, which might be due to the presence of an off-ramp followed by a lane drop further downstream of the steady area (at Ashby Avenue). The transition lengths for the remaining near-stationary zones are tabulated in Table 3.2.

Table 3.2: Summary of Changes in Traffic Variables and Respective Transition Lengths

\begin{tabular}{ccccccc}
\hline $\begin{array}{c}\text { Region } \\
\text { ID }\left(\mathbf{t}_{\mathbf{i}}\right)\end{array}$ & $\begin{array}{c}\text { Change in } \\
\text { flow } \\
(\mathbf{v p h})\end{array}$ & $\begin{array}{c}\text { Length of } \\
\text { transition } \\
(\mathbf{m})\end{array}$ & $\begin{array}{c}\text { Change in } \\
\text { Density } \\
(\mathbf{v e h s} / \mathbf{k m})\end{array}$ & $\begin{array}{c}\text { Length of } \\
\text { transition } \\
(\mathbf{m})\end{array}$ & $\begin{array}{c}\text { Change in } \\
\text { Speed } \\
(\mathbf{k m} / \mathbf{h r})\end{array}$ & $\begin{array}{c}\text { Length of } \\
\text { transition } \\
(\mathbf{m})\end{array}$ \\
\hline $\mathrm{t}_{1}$ & 1117 & 90 & 22 & 120 & 3.70 & 150 \\
$\mathrm{t}_{2}$ & 748 & 60 & 27 & 120 & 3.30 & 120 \\
$\mathrm{t}_{3}$ & 939 & 120 & 67 & 90 & 6.45 & 210 \\
$\mathrm{t}_{4}$ & 914 & 90 & 52 & 120 & 5.84 & 210 \\
$\mathrm{t}_{5}$ & 1053 & 90 & 40 & 150 & 8.87 & 210 \\
$\mathrm{t}_{6}$ & 729 & 60 & 28 & 120 & 4.78 & 150 \\
$\mathrm{t}_{7}$ & 949 & 60 & 62 & 90 & 4.26 & 150 \\
$\mathrm{t}_{8}$ & 1117 & 90 & 53 & 150 & 9.62 & 180 \\
$\mathrm{t}_{9}$ & 1414 & 90 & 50 & 120 & 10.65 & 240 \\
$\mathrm{t}_{10}$ & 779 & 90 & 47 & 90 & 3.35 & 120 \\
$\mathrm{t}_{11}$ & 705 & 60 & 63 & 90 & 4.78 & 180 \\
\hline
\end{tabular}

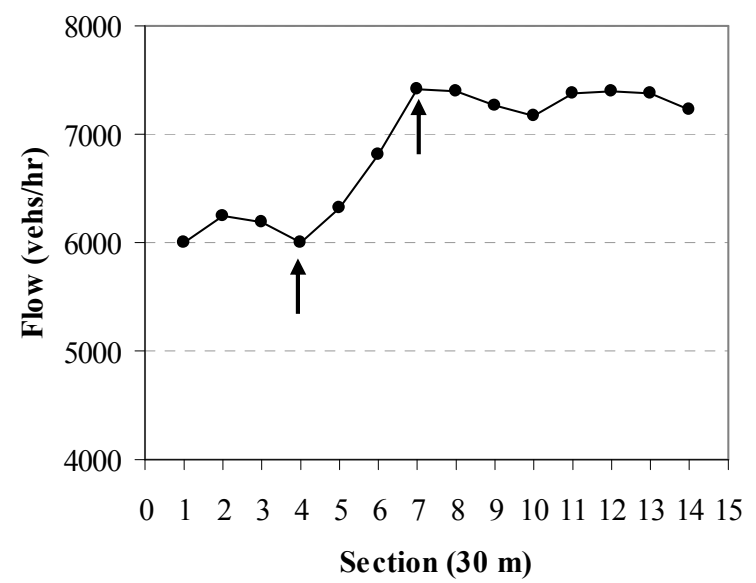

(a)

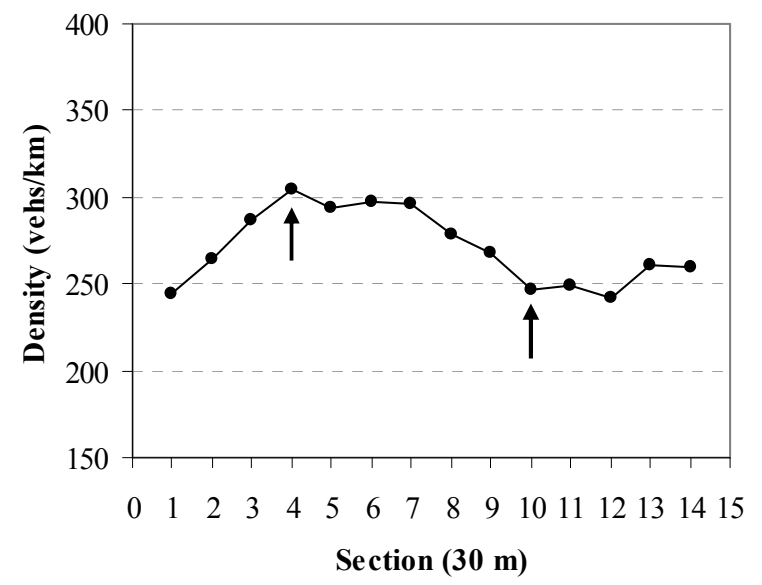

(b) 


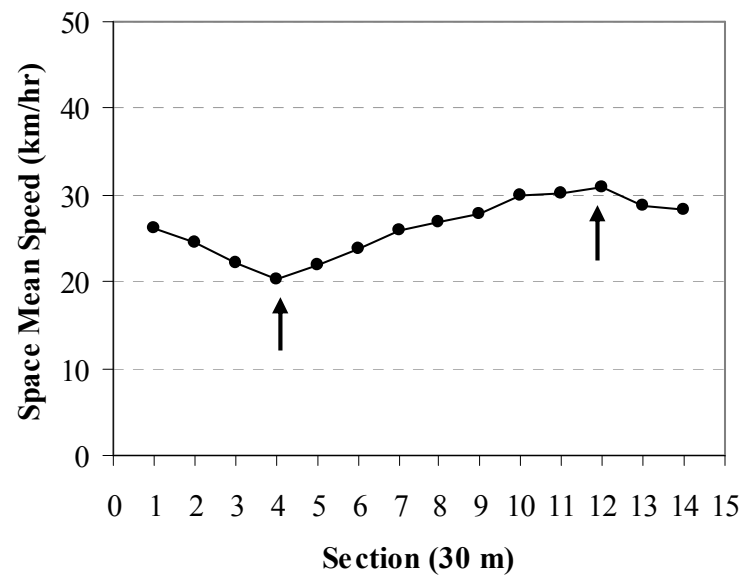

(c)

Figure 3.5: Evolutions of Traffic Variables over Freeway Sections in Stationary Period, $\mathrm{t}_{9}$ (a) Flow vs. freeway section; (b) Density vs. freeway section; (c) space-mean speed vs. freeway section

From Table 3.2, it appears that transition length varies over different stationary periods. In order to find the origin of these variations and their relation with traffic variables, the length of transitions vs. each variable is plotted, as presented in Figures 3.6. The plots reveal that the length of transition depends on the change in speed (Figure 3.6(c)), while the effects of change in flow and density appear negligible. However, this preliminary observation could not be tested statistically since the sample size is small. Thus, a more systematic evaluation is left for future investigation.

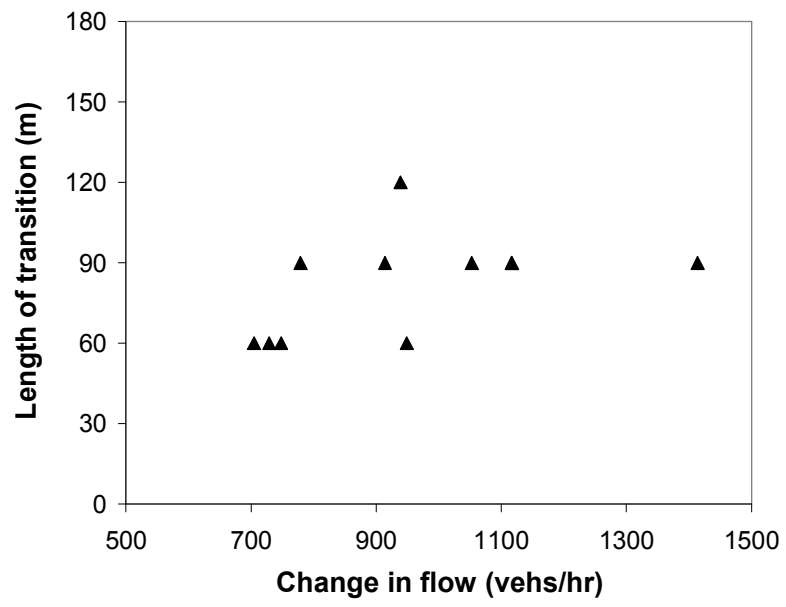

(a)

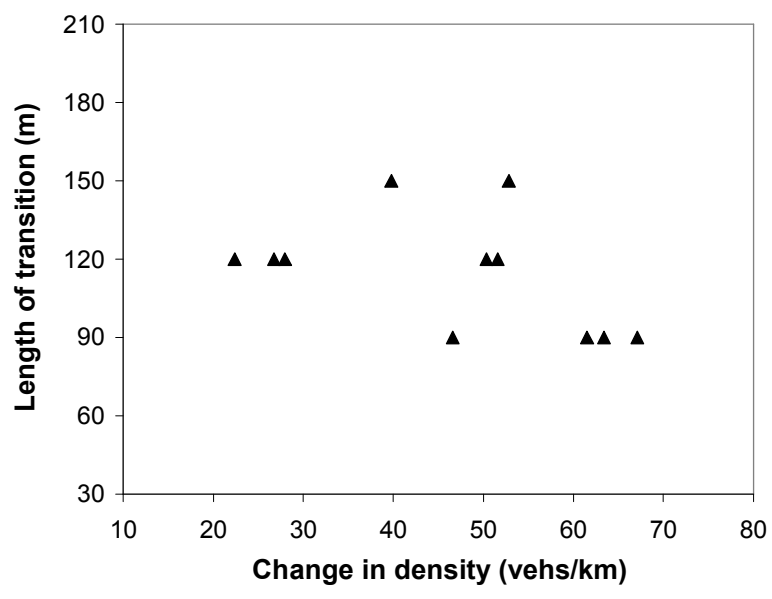

(b) 


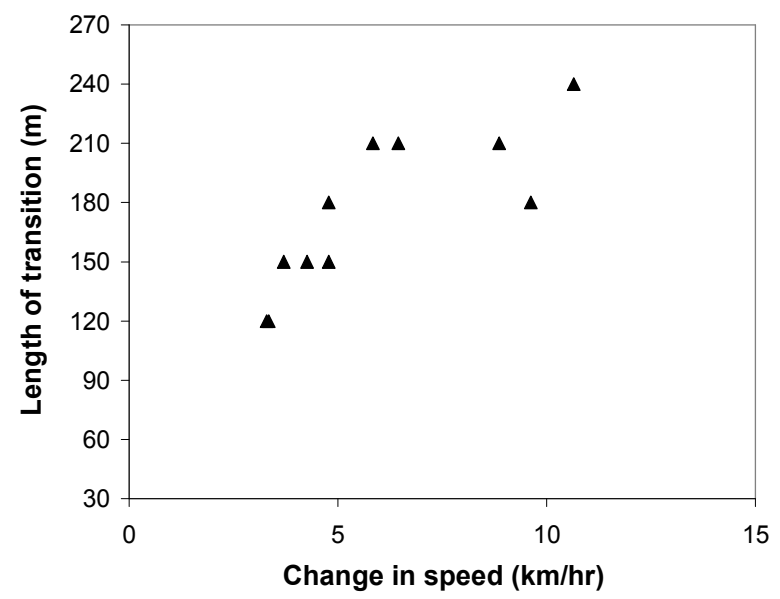

(c)

Figure 3.6: Effects of Traffic Variables on Length of Transition

(a) Length of transition vs. change in flow; (b) Length of transition vs. change in density; (c) Length of transition vs. change in space-mean speed

Finally, the spatial evolution of flow and density is traced on a flow-density plane, as shown in Figure 3.7. The circular data points correspond to the 10-second flow-density relationship averaged over the 14 segments. The scatter displays a linear trend, and the equation of a linear model (equilibrium relationship) is presented in the figure. The parameter of the linear term, $19.586 \mathrm{kmph}$, represents the speed of backward-moving kinematic waves. The estimated value is consistent with the values reported in numerous empirical studies, such as Cassidy and Mauch (2001). Moreover, the $x$-intercept of the equation yields a jam density of about $120 \mathrm{vehs} / \mathrm{km}$, which is close to the other reported values as well (e.g., Chiabaut et al. 2008a).

The data points of dark triangles in the figure represent the flow-density relations from segments 4 to 14 . These are averages over all 11 stationary periods. The plot illustrates that the increase in flow due to on-ramp inflow is considerable from sections 4 to 7 while the density is nearly constant. This movement over space is marked by the vertical arrow labeled as ' 1 ' in the figure. The opposite feature is observed downstream of section 4, such that the flow remains nearly constant while the density gradually decreases and converges to the equilibrium line (see the horizontal arrow labeled as ' 2 '). This is consistent with the observations made from Figure 3.5. 


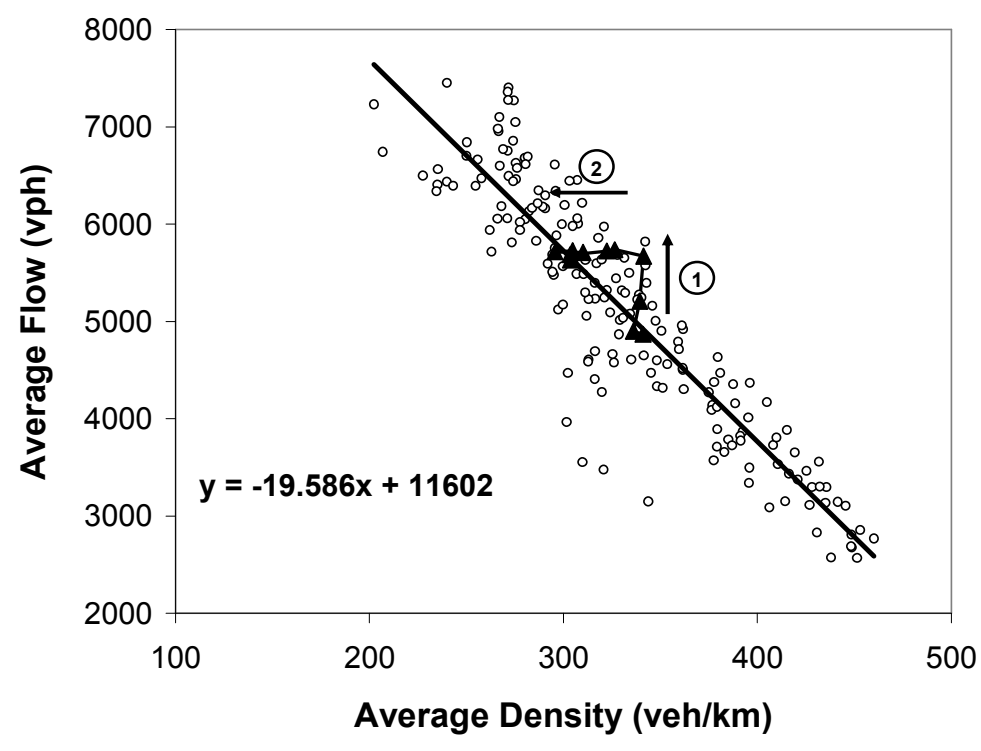

Figure 3.7: Spatial Evolution of Flow and Density. 


\subsection{TRANSITION ALONG SHOCK WAVES}

\subsection{INTRODUCTION}

Shock waves are generated whenever there is a change in existing traffic conditions. A shock wave that arises at the onset or clearance of a queue defines the tail-end of a queue, which separates a queued state from a freely flowing state. This section describes the characteristics of traffic transitions that arise as the queue grows and recedes with increasing and decreasing demands by using loop-detector data from different sites. In particular, transition durations and rates are measured along the freeway in the direction of queue propagation to observe any changes in relation to various geometric and traffic features.

\subsection{OBJECTIVES}

The primary objective is to analyze transition characteristics along shock waves during queue onset and dissipation. In particular, transition durations and rates over a freeway stretch along homogenous and inhomogeneous sections are examined. Queue formation (onset) and dissipation (recovery) phenomenon are analyzed separately. The effects of various explanatory variables such as initial and ending speeds, change in speeds, and the distance from a bottleneck on the dynamic transitions (duration/rates) are studied in detail.

\subsection{STUDY SITES AND DATA}

Four freeways sites in the U.S. and Europe were selected to analyze the transitions along shock waves. Each study site and the data obtained are described below.

\section{M4 Eastbound, U.K.}

Figure 4.1 shows a schematic of a four-kilometer stretch of M4 located in the outskirts of London as it was configured in 1998. Lanes are numbered started from the left. A recurrent bottleneck is at the lane-reduction, and the resulting queue persisted for several hours. The queues typically spilled over beyond station 7 , which corresponded to a queue length of about two kilometers. This site is interesting in that there are no on- or off-ramps and flow is conserved throughout the site. Thus, it was ideal to study how the structure of a transition changes over space in the absence of exogenous factors. Event-based speed data obtained from detector stations 4-7 are analyzed for this purpose. Of note, station 3 is omitted in the analysis since it is close to the lane-drop, and the behavior in lane 3 may be different from other upstream loops. The data were collected for 23 weekdays in November and December 1998. 


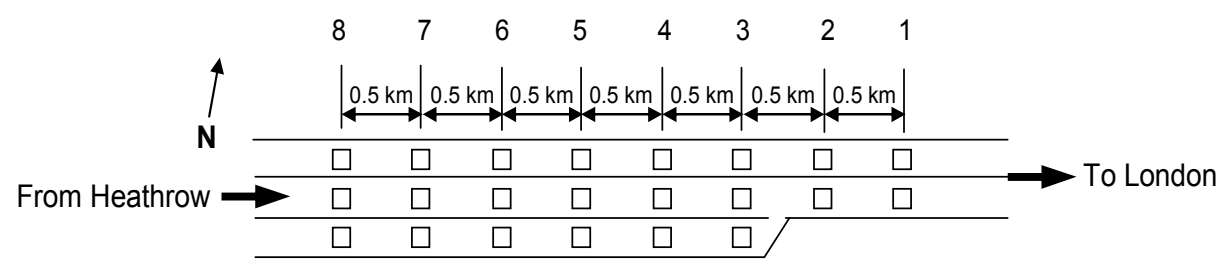

Figure 4.1: Schematic of M4 eastbound, London, U.K.

\section{A4, Netherlands}

Our second site is a three-kilometer section of northbound A4, which connects Den Haag and Amsterdam (see Figure 4.2 for a schematic). A bottleneck is located further downstream of the off-ramp (near Leiden), and the resulting queue fills the entire study section. Vehicles counts and time-mean speeds at one-minute sampling intervals were collected from 12 weekdays in January through May 2008. Similar to M4, this site is suitable to study transition structure with respect to the distance from the bottleneck. Locations 2-7 were selected for the analysis. Location 1 is omitted due to the proximity to the off-ramp in an effort to reduce any exogenous effect by the ramp.
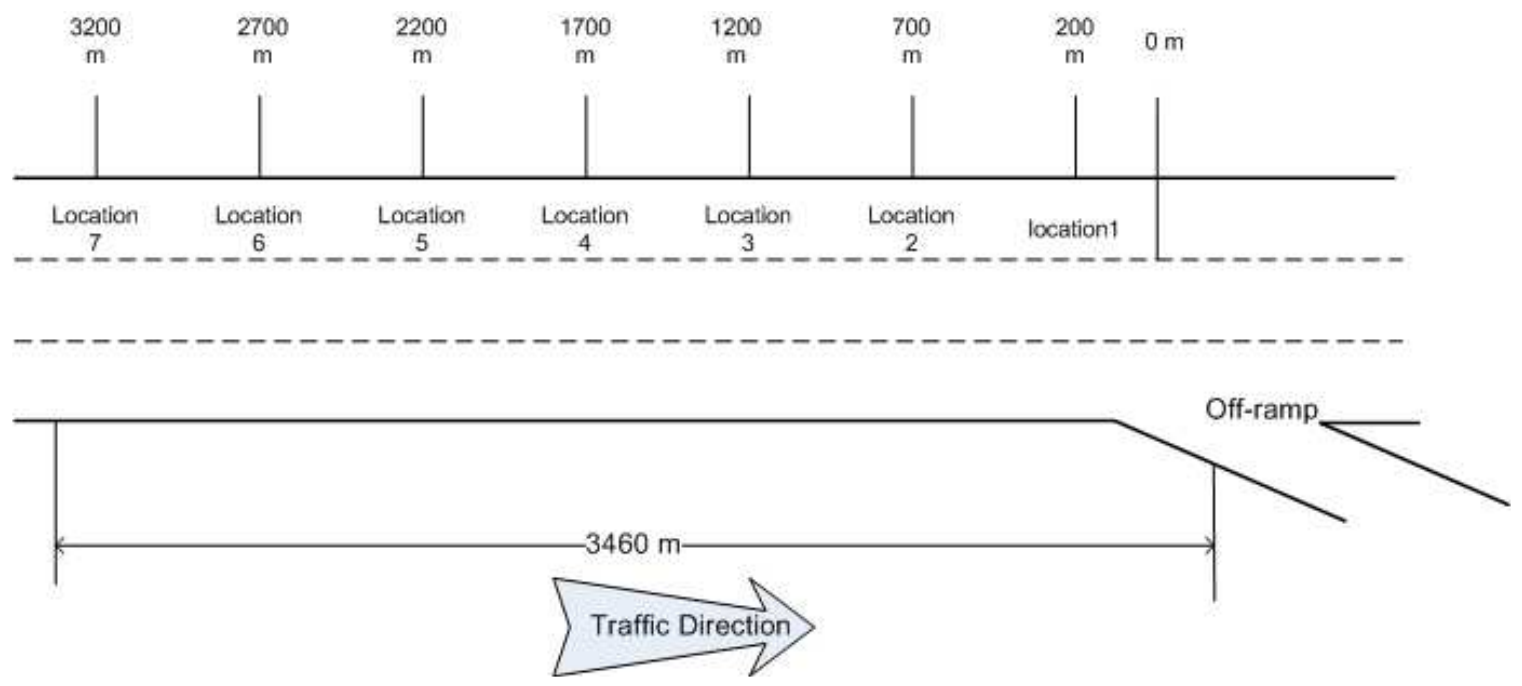

Figure 4.2: Schematic of A4, Netherlands

\section{Interstate 80, California, U.S.A.}

Traffic data from the second site, shown in Figure 4.3, were collected from a six-kilometer stretch of eastbound I-80 near San Francisco. A major recurrent bottleneck (as labeled in the figure) activates during afternoon rush periods, and the resulting queues fill the general-purpose lanes for several hours. As shown in the figure, the six-lane facility becomes five-lane facility immediately downstream of the Ashby off-ramp. The left farthest lane is designated for highoccupancy vehicles during rush hours. There are eight loop detector stations throughout the stretch, as shown in the figure. The detectors are located in each travel lane at intervals of about 
0.5 kilometers. Time-mean speeds with 30 -second sampling intervals were used to measure conditions during transition. Transitions were analyzed from stations 5-8 as a queue often starts near the merge at Ashby Avenue before a downstream queue arrives. This site is suitable to study the effect of merges and diverges on transition durations. Twenty-nine queues from JulySeptember 2002 were analyzed.

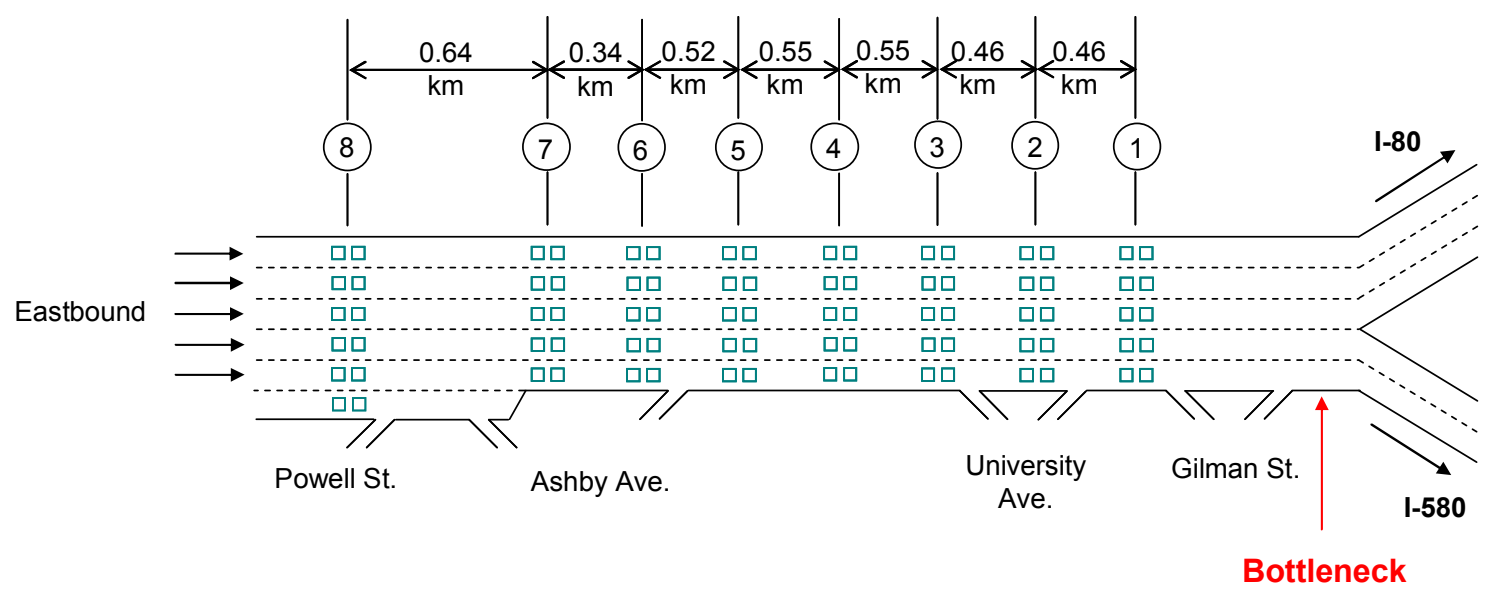

Figure 4.3: Schematic of eastbound I-80, California, U.S.A.

\section{Interstate 5, Portland, Oregon}

Figure 4.4 shows a two-kilometer stretch of northbound I-5 near Portland, Oregon. A major recurrent bottleneck is located downstream of the section shown, and all three locations are queued during afternoon rush periods. Time-mean speeds were collected by 20 -second aggregated intervals by loop detector to measure conditions during transition. This site is also suitable to study the effects of merges and diverges on transition durations. Eleven queues from July-September 2002 were analyzed.

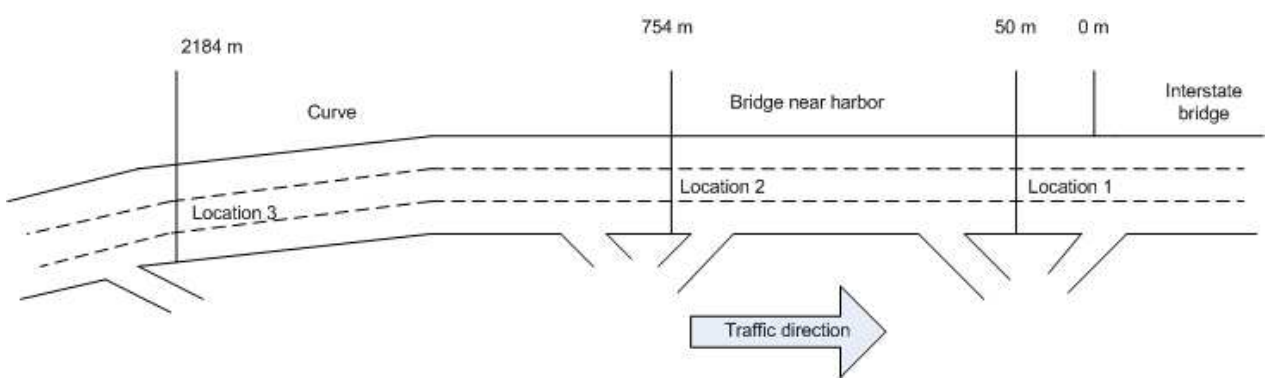

Figure 4.4: Schematic of eastbound I-5, Oregon, U.S.A. 


\subsection{METHODOLOGY}

This chapter aims to study the transition characteristics along shock waves by analyzing the relationship between the transition duration or rate and traffic parameters such as speed (before and after transition), changes in speed and distance from a bottleneck.

\section{Definitions:}

- Transition duration is defined as the time (in minutes) taken for the traffic stream to change from free-flow state to congested state or vice-versa at the onsets and dissipations of queues.

- Transition rate is defined as the rate of speed change (in $\mathrm{kmph} / \mathrm{min}$ ) during transition and can be calculated as the ratio of speed change to the transition duration.

\subsubsection{Data Processing}

The data from M4 eastbound are event-based speed data. For more efficient handling of data, the event-based speeds at each loop detector station were aggregated at 30-second sampling intervals for all 23 weekdays. Since the data from A4, I-80 and I-5 are obtained in sampling intervals of 60,30 and 20 seconds, the data can be directly used for further analysis. Example plots of speed vs. time in Figure 4.5 reveal the congestion patterns (a.m. and p.m. peaks) at the I-80 site.

According to these plots, transition into congestion emerges around 2:45 p.m. and cleared around 7:15 p.m. The congested state persisted from around 3 p.m. to 7 p.m. Other sites display similar patterns in the mornings or afternoons.
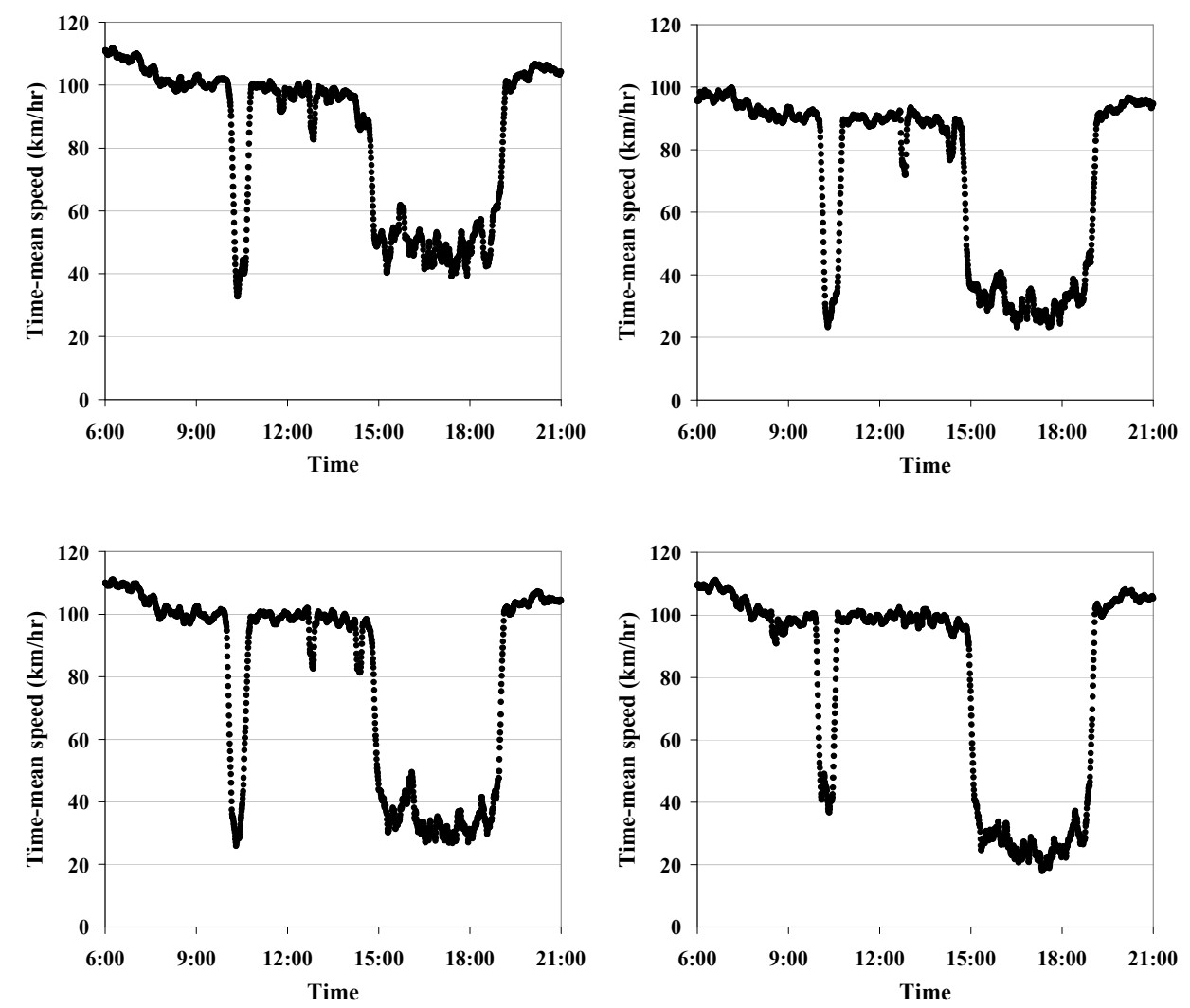
Figure 4.5: Example speeds vs. time at I-80, August 6, 2002

(a) Loop 5; (b) Loop 6; (c) Loop 7; (d) Loop 8

Speed contour plots help to gain an insight into the prevailing traffic conditions at each freeway site over time and space. These plots show the speed distributions and congestion patterns (speed transitions) from free-flow to congestion and vice versa as the queue grows (or recedes) with increasing (or decreasing) demand. As described in section 3, time is shown on the x-axis, loop detector locations are shown on the y-axis, and time-mean speeds are represented in grey scale. As noted in the legend, darker shades represent lower speeds and lighter shades represent higher speeds. Figure 4.6 presents an example of the speed contours for M4 on a single day. The queue formation, dissipation and movement can be observed from this plot. On this particular day, a queue formed downstream of the lane drop and propagated past station 7. It clearly depicts the propagation of the queue as well as the queue length.

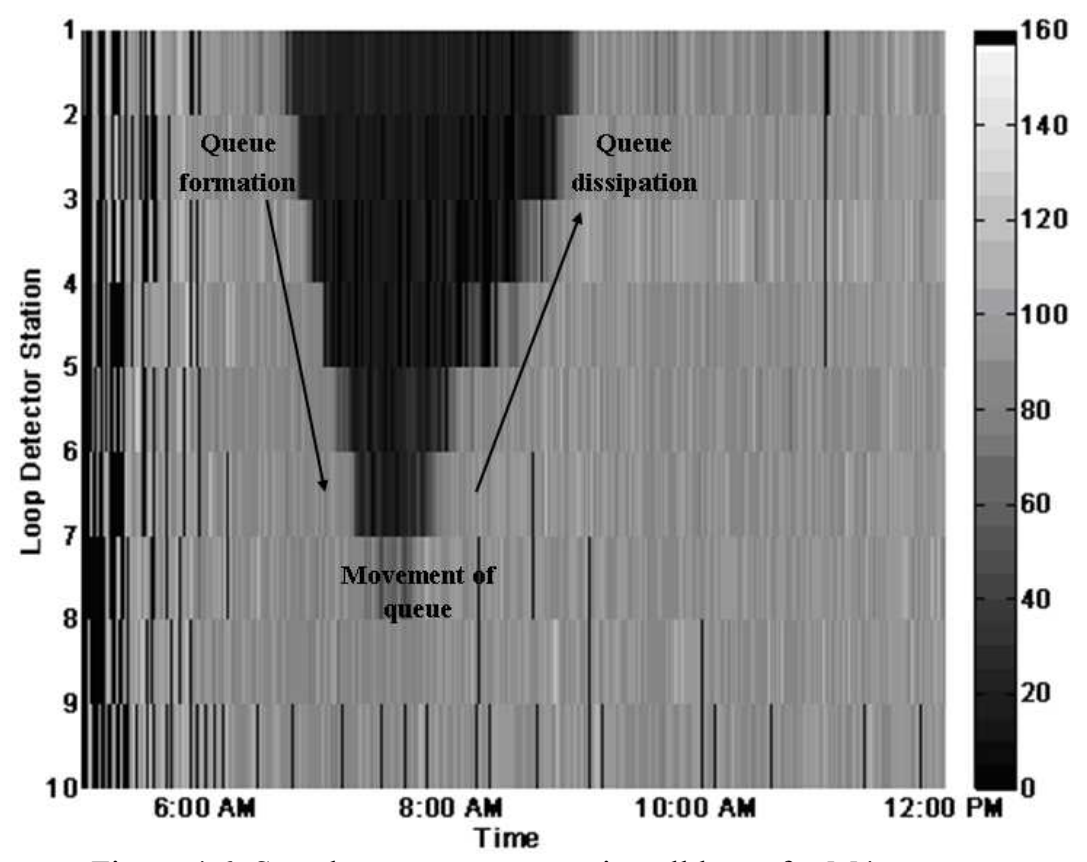

Figure 4.6: Speed contours representing all lanes for M4

\subsubsection{Measurement of Variables}

This section describes the variables used to quantify the transition as traffic flow changes from one state to another. Data are obtained from individual lanes at multiple detector locations over which queues propagate. The transition periods are identified in conjunction with speeds before and after a regime change and other relevant features (e.g., number of lanes, loop station, wave speed, etc). The intent is to build a database for various statistical analyses with dependent and explanatory variables such as transition duration, transition rate, initial speed, ending speed, 
change in speed and shock wave speed. The methodology used to obtain these variables is described in this section.

\section{Transition duration}

Transition duration is measured using time-mean speed data. It is measured as the time in minutes for the traffic to change from free-flow state to congested state or vice versa. In order to measure this, transition start and end times are identified first. At each loop detector station, fiveminute moving-average speeds are used to estimate these times more accurately so the noise in the data is reduced. An example of moving-average speeds is presented in Figure 4.7. It is notable that transition during the onset of a queue is marked by continual decreases from freeflowing to congested speeds. Similarly, transition during recovery of the queue is marked by continual increases from congested to free-flow speeds. The start and end points for the onset and recovery of a queue are marked as $a, b, c$, and $d$ in Figure 4.7. The corresponding speeds are marked as $v_{a}, v_{b}, v_{c}$ and $v_{d}$ and $t$ represents the threshold speed of congestion.

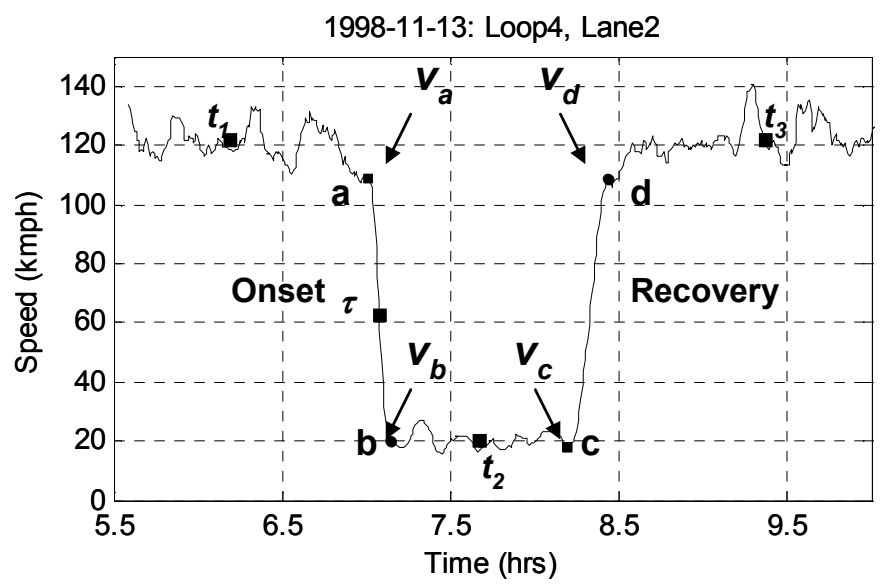

Figure 4.7: Five-Minute Moving-Average Speeds with Start and End Points of Transition for M4.

Transition start and end times for queue onset and recovery are determined in an automated fashion to identify these times in a consistent manner from day to day and reduce data extraction time. The basic principle of the algorithm implemented in MATLAB is provided below.

1. Identify the location of a recurrent bottleneck using speed contours.

2. Compute five-minute moving-average speeds for each loop station upstream of the bottleneck, and store the values in matrices (e.g., row: time, column: loop stations).

3. Identify two time points sometime before congestion begins (labeled as $t_{1}$ in Figure 4.7) and sometime during congestion (labeled as $t_{2}$ ) so that transition start and end times during the onset of congestion are included. For the clearance of congestion, we pick times during congestion and after congestion clears (labeled as $t_{2}$ and $t_{3}$, respectively). These times are determined based on the general characteristics of congestion in each site. The intent is to narrow the search for faster processing and focus on the recurrent congestion. Visual inspection of speed curves on different days reveal that congestion 
start and end times are quite consistent from day to day (within an hour or so). Nonrecurrent congestion was not considered in this study in an effort to exclude exogenous factors that may be absent in recurrent congestion.

4. Determine the initial point of search. This point was set to be the time at which the speed was the nearest to the midpoint of free-flow and typical average congested speed. The free-flow speed and congested speed are determined for each lane, loop, and site based on a subsample of data. For M4, as shown in Figure 4.7, the free-flow and average congested speeds are respectively $120 \mathrm{~km} / \mathrm{hr}$ and $20 \mathrm{~km} / \mathrm{hr}$. Thus, the initial search point (labeled as $\tau$ in Figure 15) corresponds to the time when the speed was closest to 70 $\mathrm{km} / \mathrm{hr}$.

5. Find transition start and end times by searching left (backward in time) and right (forward in time), respectively, from the initial search point.

6. The criteria to stop the searches are:

a. Start time of transition: continue the search as long as the incremental speed change (backward in time) is positive or the speed is less than free-flow speed. To account for noise in the data, negative incremental speed changes are permitted as long as they are not observed consecutively during a one-minute period.

b. End time of transition: continue the search as long as the incremental speed change is negative or the speed is above the threshold. Positive incremental speed changes are permitted as long as they are not observed consecutively during a one-minute period.

After identifying the start time and the end time, the difference gives the transition duration,

$$
\text { Transition duration, } t=t_{e}-t_{i} \text { (minutes) }
$$

where, $t_{i}:$ time in minutes at transition start point $(a$ or $c$ )

$t_{e}:$ time in minutes at transition end point $(b$ or $d)$

\section{Initial and ending speeds}

The initial speeds $\left(v_{a}\right.$ and $\left.v_{c}\right)$ are measured as the five-minute average speeds in $\mathrm{km} / \mathrm{hr}$ at the start of transition (i.e., the speed at point $a$ during the onset and point $c$ during the recovery of a queue, as shown in Figure 4.7). Similarly, the ending speeds $\left(v_{b}\right.$ and $\left.v_{d}\right)$ are measured as the fiveminute average speeds at the end of transition (i.e., the speed at points $b$ and $d$ during the onset and recovery, respectively).

\section{Change in speed}

The change is speed is measured as the difference between initial and ending speeds.

$$
\text { Change in speed, } \Delta v=v_{b}-v_{a} \text { or } v_{d}-v_{c}(\mathrm{~km} / \mathrm{hr})
$$

Thus, the change in speed is negative during onsets and positive during dissipations.

\section{Transition rate}

The transition rate measures the amount of speed reduction expected within unit time (minutes). Thus, it is measured as the ratio of speed reduction to the transition duration in minutes. 


$$
\text { Transition rate, } r_{T}=\frac{\Delta v}{T} \quad(\mathrm{kmph} / \mathrm{min})
$$

\subsection{ANALYSIS}

Once the relevant variables are measured, transition characteristics along shock waves during queue onsets and dissipations are studied separately. The dependent variables and potential explanatory variables considered in this study are as follows:

- Dependent variables: transition duration or transition rate

- Explanatory variables:

- Loop: loop detector stations are correlated with distance from bottlenecks and are used as a proxy

- Lane: lane numbers are used to study the differences in lane-wise behavior

- Initial Speed, $v_{i}(\mathrm{~km} / \mathrm{hr})$

- Ending Speed, $v_{e}(\mathrm{~km} / \mathrm{hr})$

- Change in Speed, $\Delta v(\mathrm{~km} / \mathrm{hr})$

\subsubsection{Onset of Queue}

Table 4.1 shows the median transition durations and rates for each lane at each site. The durations range from 10 to 25 minutes, marking transition rates of -7.6 to $-2.2 \mathrm{kmph} / \mathrm{min}$ depending on the site. These values varied from site to site, such that the durations at M4 and A4 are smaller than those at I-80 and I-5 by comparison. However, after accounting for the amount of speed reduction, M4 displays the largest magnitude of transition rates while I-5 displays the lowest transition rate. The durations and rates seem to be affected by factors other than geometry as there is little correlation between number of lanes and duration (or rate) over different sites. This study focuses more on the factors, such as amount of speed reduction and distance from a bottleneck, which may change the transition characteristics within each site.

Table 4.1: Median Transition Durations and Rates at Four Study Sites

\begin{tabular}{lcccccccc}
\hline & \multicolumn{3}{c}{ Transition duration $(\mathbf{m i n s})$} & \multicolumn{4}{c}{ Transition rate $(\mathbf{k m p h} / \mathbf{m i n})$} \\
\cline { 2 - 9 } & M4 & A4 & $\mathbf{I - 8 0}$ & $\mathbf{I - 5}$ & $\mathbf{M} 4$ & $\mathbf{A 4}$ & $\mathbf{I - 8 0}$ & $\mathbf{I - 5}$ \\
\hline Lane 1 & 10.5 & 11.5 & - & 25.0 & -7.0 & -5.2 & - & -2.8 \\
Lane 2 & 11.5 & 11.0 & 19.5 & 23.0 & -7.6 & -4.6 & -3.5 & -2.6 \\
Lane 3 & 14.0 & 11.0 & 22.5 & 25.0 & -6.9 & -4.0 & -2.7 & -2.2 \\
Lane 4 & - & - & 21.5 & - & - & - & -2.5 & - \\
Lane 5 & - & - & 19.8 & - & - & - & -2.6 & - \\
\hline
\end{tabular}

\subsubsection{Correlation Coefficients}

All the explanatory variables are tested for correlations among them to identify a set of orthogonal variables. The correlation coefficients and the corresponding $p$-values for the 
onsets of queues on all four sites are displayed in Tables $4.2-4.5$. The shaded $p$-values indicate that the correlations of the corresponding pairs are significant. It is notable that some of the speed-related measurements $\left(v_{i}, v_{e}\right.$ and $\left.\Delta v\right)$ correlate with one another as well as with Lane for all four sites. This implies lane-specific transition characteristics in terms of initial and ending speeds and changes in speed during transition. It is also notable that all speed-related variables are correlated with Loop for the I-80 and I-5 sites. This is attributable to the presence of on- and off-ramps in the study sections, where entering and exiting flows delineate traffic states upstream and downstream of the interchanges. In light of the finding, the effects of lane, loop (distance from a bottleneck) and ramps are investigated separately through the parametric one-way ANOVA and the non-parametric Kruskal-Wallis test.

Table 4.2: Correlation Coefficients among Explanatory Variables: M4 (Onset)

\begin{tabular}{ccccccccccc}
\hline & \multicolumn{4}{c}{ Correlation Coefficient } & \multicolumn{5}{c}{ p-value } \\
\cline { 2 - 12 } & Lane & Loop & $\boldsymbol{v}_{\boldsymbol{i}}$ & $\boldsymbol{v}_{\boldsymbol{e}}$ & $\Delta \boldsymbol{v}$ & Lane & Loop & $\boldsymbol{v}_{\boldsymbol{i}}$ & $\boldsymbol{v}_{\boldsymbol{e}}$ & $\Delta \boldsymbol{v}$ \\
\hline Lane & 1.00 & 0.01 & 0.81 & 0.24 & -0.71 & - & 0.85 & 0.00 & 0.00 & 0.00 \\
Loop & 0.01 & 1.00 & -0.01 & 0.16 & 0.10 & 0.85 & - & 0.78 & 0.00 & 0.06 \\
$\boldsymbol{v}_{\boldsymbol{i}}$ & 0.81 & -0.01 & 1.00 & 0.20 & -0.85 & 0.00 & 0.78 & - & 0.00 & 0.00 \\
$\boldsymbol{v}_{\boldsymbol{e}}$ & 0.24 & 0.16 & 0.20 & 1.00 & 0.15 & 0.00 & 0.00 & 0.00 & - & 0.01 \\
$\Delta \boldsymbol{v}$ & -0.71 & 0.10 & -0.85 & 0.15 & 1.00 & 0.00 & 0.06 & 0.00 & 0.01 & - \\
\hline
\end{tabular}

Table 4.3: Correlation Coefficients among Explanatory Variables: A4 (Onset)

\begin{tabular}{ccccccccccc}
\hline & \multicolumn{4}{c}{ Correlation Coefficient } & \multicolumn{5}{c}{ p-value } \\
\cline { 2 - 12 } & Lane & Loop & $\boldsymbol{v}_{\boldsymbol{i}}$ & $\boldsymbol{v}_{\boldsymbol{e}}$ & $\Delta \boldsymbol{v}$ & Lane & Loop & $\boldsymbol{v}_{\boldsymbol{i}}$ & $\boldsymbol{v}_{\boldsymbol{e}}$ & $\Delta \boldsymbol{v}$ \\
\hline Lane & 1.00 & 0.02 & -0.84 & -0.23 & -0.33 & - & 0.81 & 0.00 & 0.00 & 0.00 \\
Loop & 0.02 & 1.00 & -0.08 & -0.10 & 0.00 & 0.81 & - & 0.24 & 0.13 & 0.96 \\
$\boldsymbol{v}_{\boldsymbol{i}}$ & -0.84 & -0.08 & 1.00 & 0.25 & 0.36 & 0.00 & 0.24 & - & 0.00 & 0.00 \\
$\boldsymbol{v}_{\boldsymbol{e}}$ & -0.23 & -0.10 & 0.25 & 1.00 & -0.61 & 0.00 & 0.13 & 0.00 & - & 0.00 \\
$\Delta \boldsymbol{v}$ & -0.33 & 0.00 & 0.36 & -0.61 & 1.00 & 0.00 & 0.96 & 0.00 & 0.00 & - \\
\hline
\end{tabular}

Table 4.4: Correlation Coefficients among Explanatory Variables: I-80 (Onset)

\begin{tabular}{ccccccccccc}
\hline & \multicolumn{4}{c}{ Correlation Coefficient } & \multicolumn{5}{c}{ p-value } \\
\cline { 2 - 12 } & Lane & Loop & $\boldsymbol{v}_{\boldsymbol{i}}$ & $\boldsymbol{v}_{\boldsymbol{e}}$ & $\Delta \boldsymbol{v}$ & Lane & Loop & $\boldsymbol{v}_{\boldsymbol{i}}$ & $\boldsymbol{v}_{\boldsymbol{e}}$ & $\Delta \boldsymbol{v}$ \\
\hline Lane & 1.00 & 0.00 & -0.66 & 0.00 & 0.22 & - & 1.00 & 0.00 & 0.93 & 0.00 \\
Loop & 0.00 & 1.00 & 0.19 & -0.58 & -0.63 & 1.00 & - & 0.00 & 0.00 & 0.00 \\
$\boldsymbol{v}_{\boldsymbol{i}}$ & -0.66 & 0.19 & 1.00 & 0.06 & -0.55 & 0.00 & 0.00 & - & 0.23 & 0.00 \\
$\boldsymbol{v}_{\boldsymbol{e}}$ & 0.00 & -0.58 & 0.06 & 1.00 & 0.65 & 0.93 & 0.00 & 0.23 & - & 0.00 \\
$\Delta \boldsymbol{v}$ & 0.22 & -0.63 & -0.55 & 0.65 & 1.00 & 0.00 & 0.00 & 0.00 & 0.00 & - \\
\hline
\end{tabular}

Table 4.5: Correlation Coefficients among Explanatory Variables: I-5 (Onset)

\begin{tabular}{ccccccccccc}
\hline & \multicolumn{4}{c}{ Correlation Coefficient } & \multicolumn{5}{c}{ p-value } \\
\cline { 2 - 11 } & Lane & Loop & $\boldsymbol{v}_{\boldsymbol{i}}$ & $\boldsymbol{v}_{\boldsymbol{e}}$ & $\Delta \boldsymbol{v}$ & Lane & Loop & $\boldsymbol{v}_{\boldsymbol{i}}$ & $\boldsymbol{v}_{\boldsymbol{e}}$ & $\Delta \boldsymbol{v}$ \\
\hline Lane & 1.00 & 0.00 & -0.41 & -0.29 & 0.08 & - & 1.00 & 0.00 & 0.00 & 0.44 \\
Loop & 0.00 & 1.00 & -0.29 & 0.46 & -0.55 & 1.00 & - & 0.00 & 0.00 & 0.00 \\
$\boldsymbol{v}_{\boldsymbol{i}}$ & -0.41 & -0.29 & 1.00 & 0.06 & 0.35 & 0.00 & 0.00 & - & 0.53 & 0.00 \\
$\boldsymbol{v}_{\boldsymbol{e}}$ & -0.29 & 0.46 & 0.06 & 1.00 & -0.78 & 0.00 & 0.00 & 0.53 & - & 0.00 \\
$\Delta \boldsymbol{v}$ & 0.08 & -0.55 & 0.35 & -0.78 & 1.00 & 0.44 & 0.00 & 0.00 & 0.00 & - \\
\hline
\end{tabular}




\subsubsection{Effect of Lane}

As a preliminary analysis, lane-wise distributions of initial speeds, ending speeds, transition durations and transition rates are compared using box plots. General observations from the box plots are verified by both parametric and non-parametric statistical tests since the samples from A4 and I-5 are relative small (11 and 12 queues, respectively), and the normality assumption may not hold well. The one-way ANOVA and the Kruskal-Wallis (K-W) tests are performed on transition durations and rates across lanes at each individual loop station.

\section{M4, U.K.}

At this freeway site (see Figure 4.1), 24 queues are analyzed to measure the transitions during queue onsets. Figures 4.8(a) and (b), respectively, show lane-wise distributions of initial speeds and changes in speed. The box plots illustrate that the initial speeds increase toward the right lanes. This is expected for a U.K. highway since the far right lane corresponds to the median lane. Once a queue forms, the magnitude of speed reduction also increases toward the right lanes. The median transition durations, shown in Figure 4.8(c), ranged from 10 to 15 minutes with an increasing trend toward the right lanes. However, the lane-specific trend is not as pronounced as the initial speeds and reductions in speed. The trend is even less pronounced for transition rates as illustrated in Figure 4.8(d). This indicates that when speed reductions are normalized by transition durations (or vice versa), transition behavior is similar across lanes. This observation is further supported by various statistical tests as explained from here on.

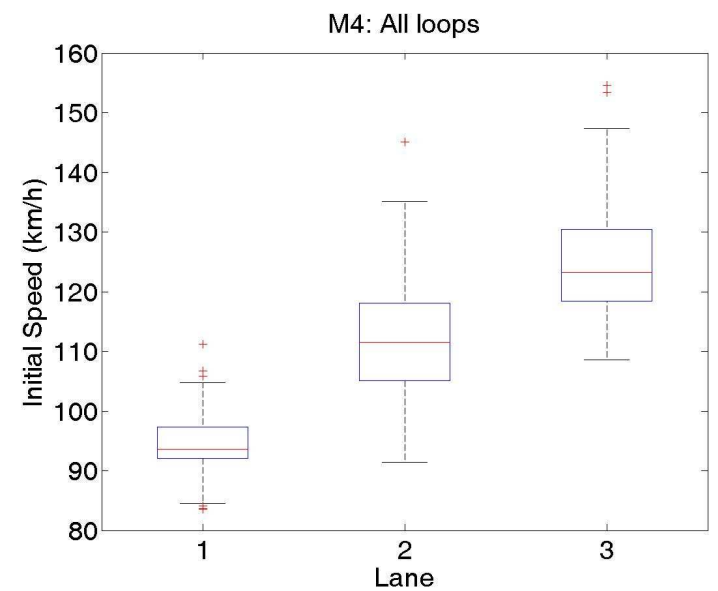

(a)

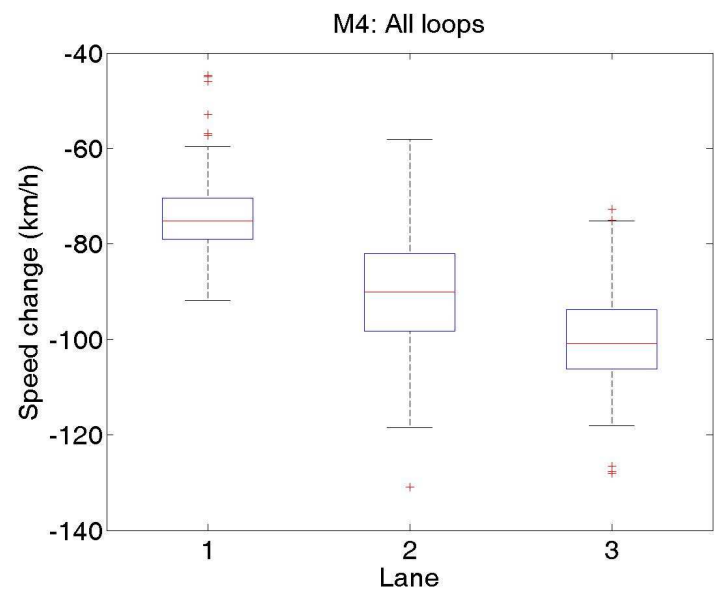

(b) 


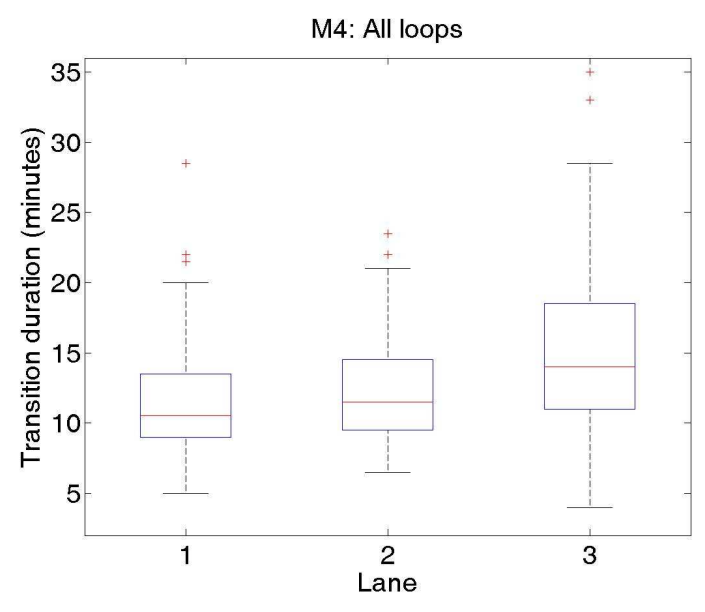

(c)

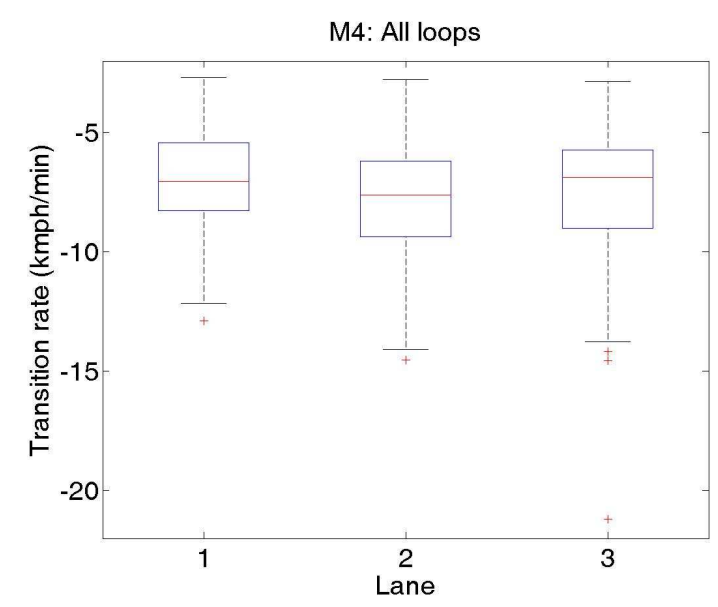

(d)

Figure 4.8: Lane-wise distribution of transition characteristics at onset of queue, M4

(a) Initial speed; (b) Change in speed; (c) Transition Duration; (d) Transition Rate

Table 4.6 shows the results from the K-W test and one-way ANOVA at each loop station. The values reported in the table are the $p$-values under the null hypothesis of the same mean (ANOVA) or median (K-W test) across lanes. Thus, at the 0.05 level, a $p$-value smaller than 0.05 indicates that at least one lane has a mean or median different from other lanes at that station. In terms of durations, the results are mixed: the differences in duration and rate across lanes are statistically insignificant at the 0.05 level at stations 5 and 6 while the differences are significant at station 7. Moreover, the two tests report contradicting results at loop station 4 . Nevertheless, the $p$-values are relatively small, indicating that lane could be a factor. To the contrary, the results for transition rates from the two tests are consistent for different loop stations. For all stations, all $p$-values are well above 0.05 , indicating that the differences in transition rate across lanes are not statistically significant. The results indicate that when traffic changes from free-flow to congested regimes, the rate of speed reduction is similar across lanes although the duration may vary due to the differences in total amount of speed reduction.

Table 4.6: Statistical Test Results for the Effect of Lane: M4 (Onset)

\begin{tabular}{ccccc}
\hline & \multicolumn{2}{c}{ Transition duration } & \multicolumn{2}{c}{ Transition rate } \\
\cline { 2 - 5 } Loop Number & K-W Test & One-way ANOVA & K-W Test & One-way ANOVA \\
\hline 4 & 0.08 & 0.04 & 0.59 & 0.66 \\
5 & 0.05 & 0.13 & 0.29 & 0.45 \\
6 & 0.16 & 0.08 & 0.22 & 0.17 \\
7 & 0.02 & 0.01 & 0.98 & 0.87 \\
\hline
\end{tabular}

\section{A4, Netherland}

At this freeway site, all stations shown in Figure 4.2 are congested during rush hours. Based on the data from 12 queues, lane-wise distributions of transition characteristics are illustrated in Figure 4.9. At this site, lane 1 (far left lane) exhibits the largest initial speeds, and the initial speeds decrease toward the right lanes. The magnitude of speed 
reductions show a similar trend, such that lane 1 displays the largest speed reduction. Transition durations and rates, however, display more subtle lane-specific behavior. This is confirmed by the K-W test and the one-way ANOVA, as presented in Table 4.7. With an exception of transition rates at station 7, the differences in transition durations and rates across lanes are statistically insignificant.

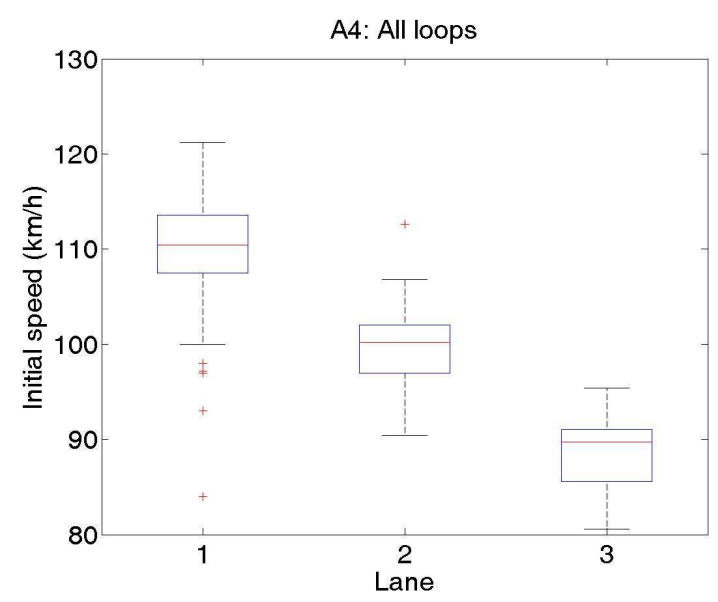

(a)

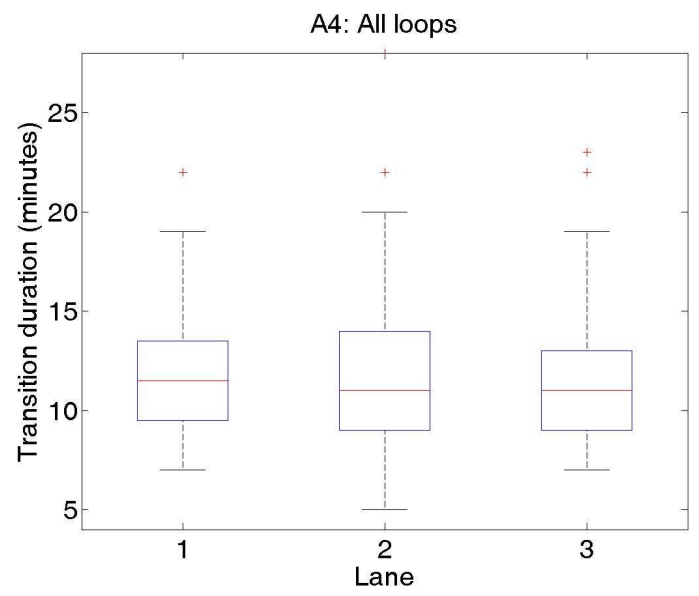

(c)

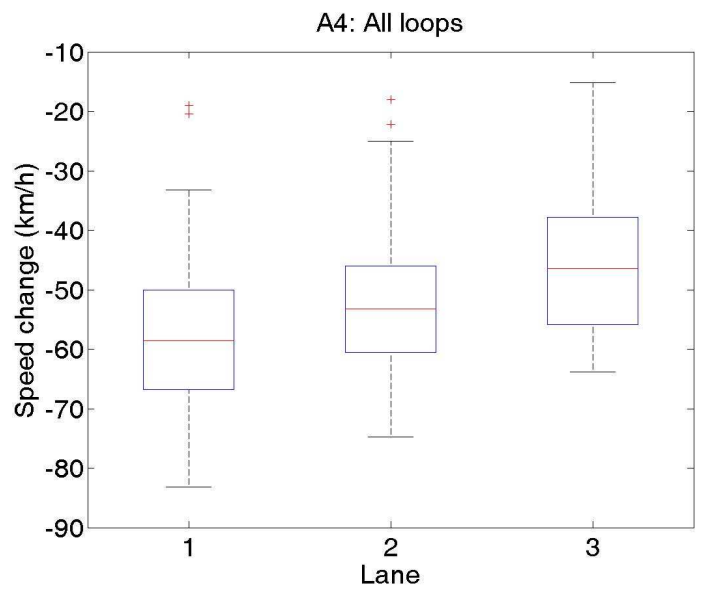

(b)

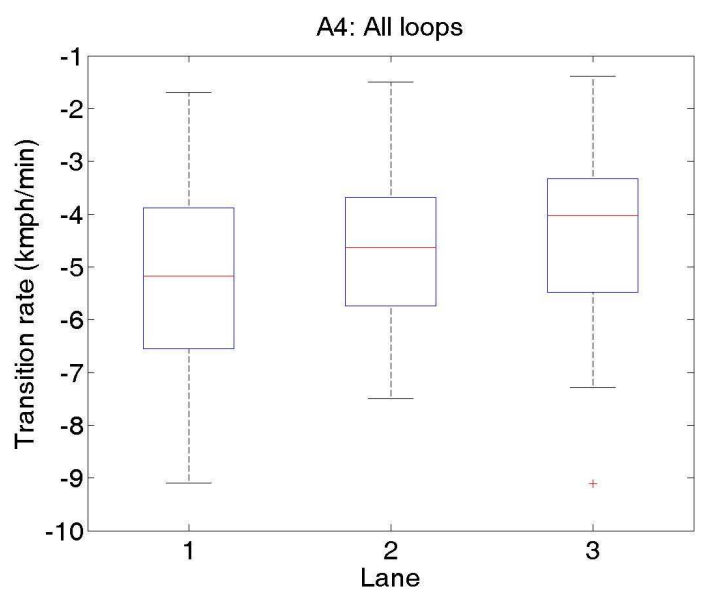

(d)

Figure 4.9: Lane-wise distribution of transition characteristics at onset of queue, A4 (a) Initial speed; (b) Change in speed; (c) Transition Duration; (d) Transition Rate

Table 4.7: Statistical Test Results for the Effect of Lane: A4 (Onset)

\begin{tabular}{ccccc}
\hline & \multicolumn{2}{c}{ Transition duration } & \multicolumn{2}{c}{ Transition rate } \\
\cline { 2 - 5 } Loop Number & K-W Test & One-way ANOVA & K-W Test & One-way ANOVA \\
\hline 2 & 0.971 & 0.819 & 0.479 & 0.357 \\
3 & 0.900 & 0.764 & 0.518 & 0.594 \\
4 & 0.775 & 0.785 & 0.290 & 0.291 \\
5 & 0.733 & 0.812 & 0.815 & 0.902 \\
6 & 0.738 & 0.667 & 0.150 & 0.079 \\
7 & 0.598 & 0.756 & 0.011 & 0.008 \\
\hline
\end{tabular}




\section{I-80, California, U.S.A.}

At this freeway site, 29 queues were analyzed. The lane-wise distributions of transition characteristics are shown in Figure 4.10. Similar to the previous sites, the initial speeds and speed change display lane-specific features, and this also is confirmed by both $\mathrm{K}-\mathrm{W}$ test and ANOVA. However, unlike the previous sites, the differences in transition rates across lanes are statistically significant at all loop stations, as shown in Table 4.8.

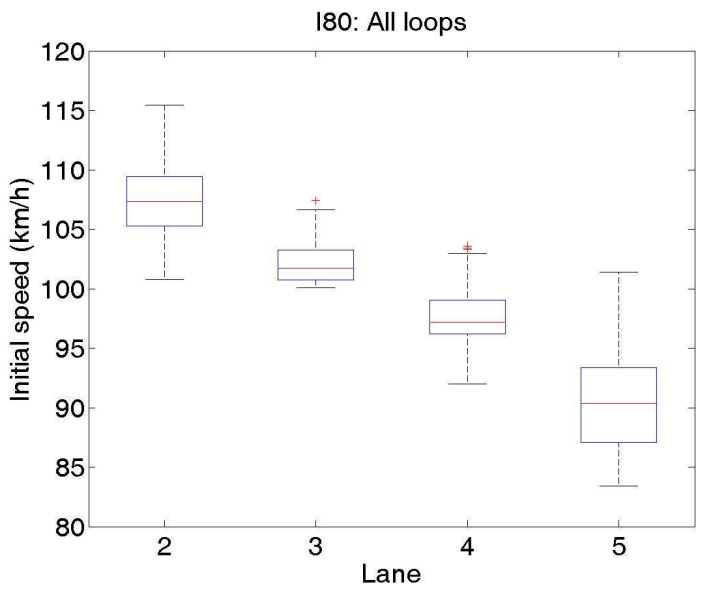

(a)

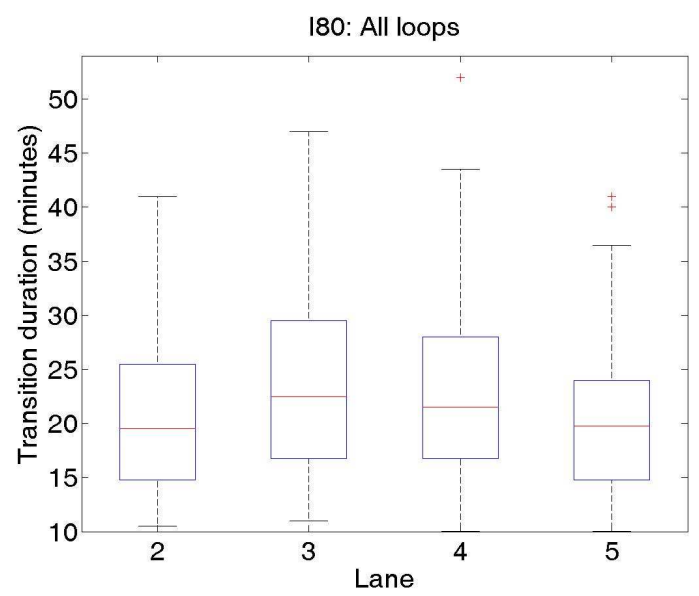

(c)

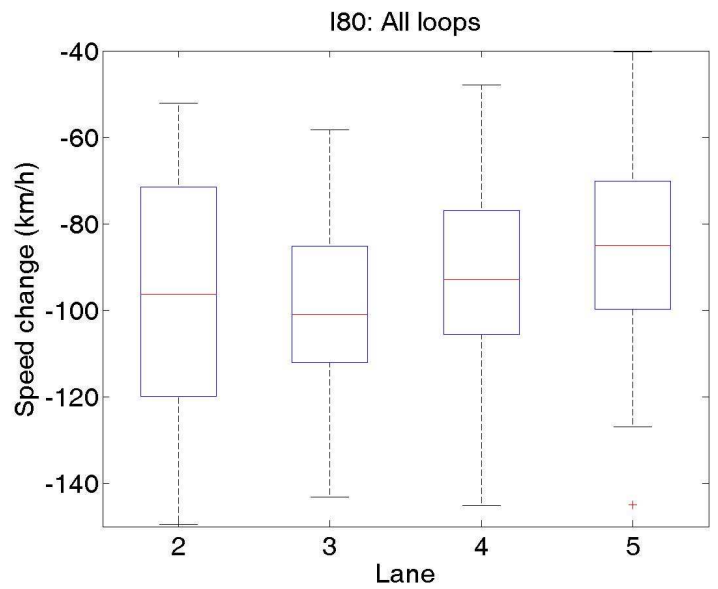

(b)

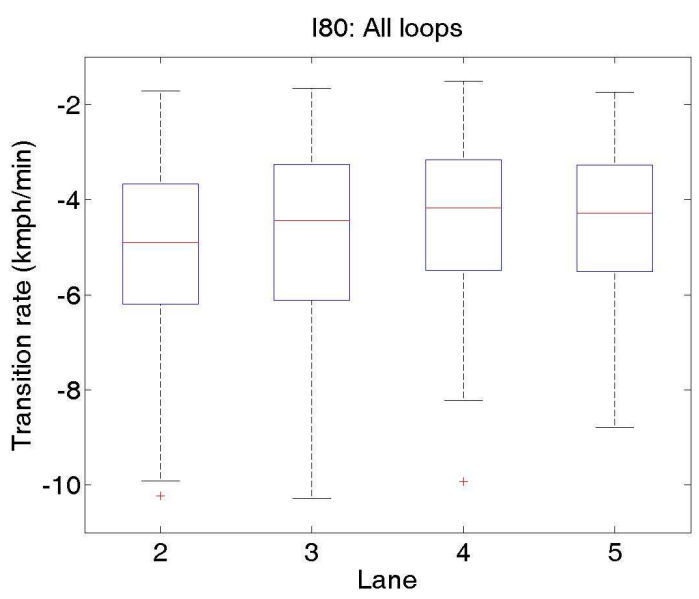

(d)

Figure 4.10: Lane-Wise Transition Duration Distributions by Detector Station for Onset of Queue, I-80

Table 4.8: Statistical Test Results for the Effect of Lane: I-80 (Onset)

\begin{tabular}{ccccc}
\hline & \multicolumn{2}{c}{ Transition duration } & \multicolumn{2}{c}{ Transition rate } \\
\cline { 2 - 5 } Loop Number & K-W Test & One-way ANOVA & K-W Test & One-way ANOVA \\
\hline 5 & 0.15 & 0.15 & 0.02 & 0.01 \\
6 & 0.02 & 0.01 & 0.00 & 0.00 \\
7 & 0.48 & 0.23 & 0.02 & 0.02 \\
8 & 0.21 & 0.06 & 0.04 & 0.04 \\
\hline
\end{tabular}




\section{I-5, Oregon, U.S.A.}

Unlike other previous sites, speed reductions (Figure 4.11(b)) during transitions are comparable across lanes. Statistical test results also support this observation. The differences in transition rates also are found statistically insignificant for most locations, as shown in Table 4.9.

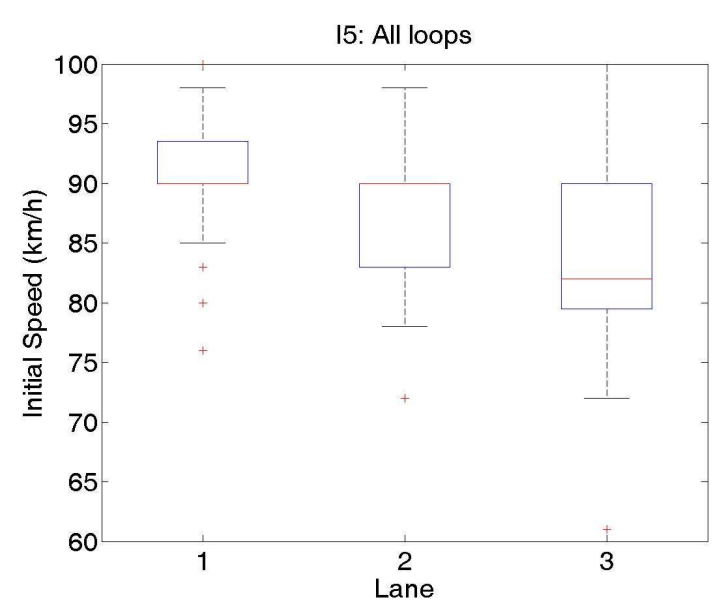

(a)

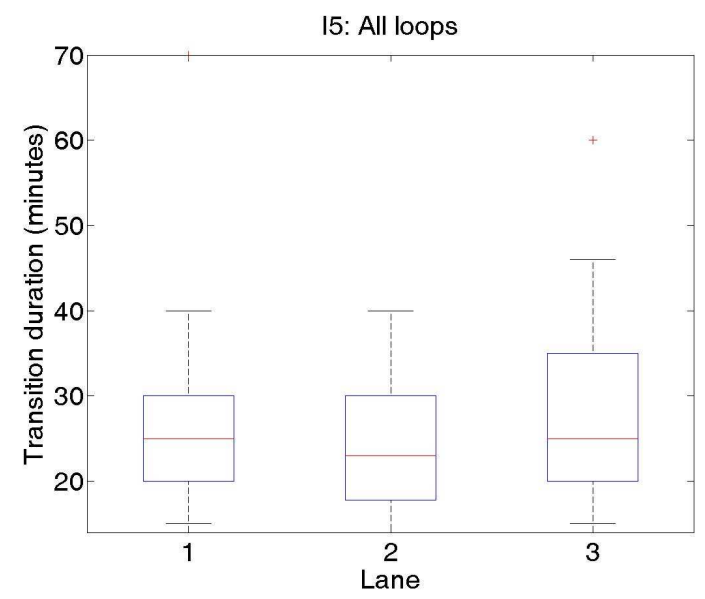

(c)

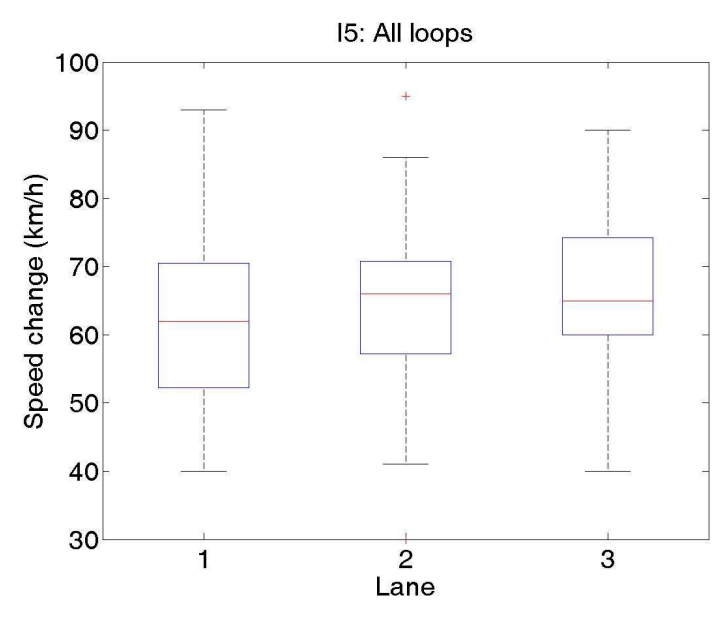

(b)

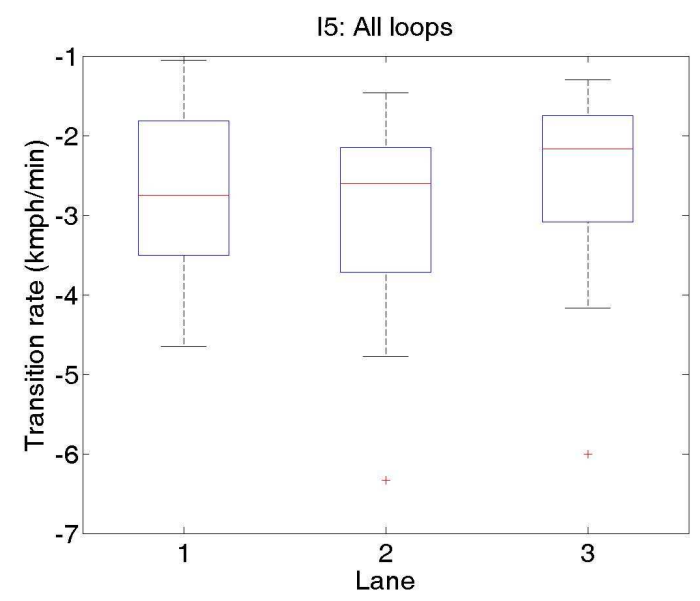

(d)

Figure 4.11: Lane-Wise Transition Duration Distributions by Detector Station for Onset of Queue, I-5

Table 4.9: Statistical Test Results for the Effect of Lane: I-5 (Onset)

\begin{tabular}{ccccc}
\hline & \multicolumn{2}{c}{ Transition duration } & \multicolumn{2}{c}{ Transition rate } \\
\cline { 2 - 5 } Loop Number & K-W Test & One-way ANOVA & K-W Test & One-way ANOVA \\
\hline 1 & 0.028 & 0.228 & 0.041 & 0.077 \\
2 & 0.352 & 0.235 & 0.399 & 0.637 \\
3 & 0.035 & 0.037 & 0.114 & 0.139 \\
\hline
\end{tabular}




\subsubsection{Effect of Distance from a Bottleneck}

This section describes how the transition durations and rates would change in the absence of ramps as queues propagate upstream. Freeway sites M4 and A4 are suitable for this study as both sites are free from intervening ramps. We perform K-W and ANOVA tests to assess statistical differences in transition durations and rates across loop stations for each lane. Of note, loop station numbers are used as a proxy for the distance from a bottleneck. The resulting $p$-values for M4 and A4 are respectively shown in Tables 4.10 and 4.11. The results show that the $p$-values are well above 0.05 in most cases, indicating that transition structure does not change without intervening ramps as a queue propagates upstream.

Table 4.10: Statistical Test Results for the Effect of Loop: M4 (Onset)

\begin{tabular}{ccccc}
\hline & \multicolumn{2}{c}{ Transition duration } & \multicolumn{2}{c}{ Transition rate } \\
\cline { 2 - 5 } Lane Number & K-W Test & One-way ANOVA & K-W Test & One-way ANOVA \\
\hline 1 & 0.44 & 0.62 & 0.72 & 0.70 \\
2 & 0.32 & 0.58 & 0.21 & 0.52 \\
3 & 0.86 & 0.37 & 0.43 & 0.93 \\
\hline
\end{tabular}

Table 4.11: Statistical Test Results for the Effect of Loop: A4 (Onset)

\begin{tabular}{ccccc}
\hline & \multicolumn{2}{c}{ Transition duration } & \multicolumn{2}{c}{ Transition rate } \\
\hline Lane Number & K-W Test & One-way ANOVA & K-W Test & One-way ANOVA \\
\hline 1 & 0.64 & 0.66 & 0.05 & 0.06 \\
2 & 0.73 & 0.59 & 0.10 & 0.10 \\
3 & 0.93 & 0.88 & 0.54 & 0.33 \\
\hline
\end{tabular}

\subsubsection{Effects of Ramps}

We now focus our attention on the effect of on- and off-ramps. Sites I- 80 and I-5 are suitable for this type of analysis since both contain on- and off-ramps. In particular, an on-ramp resides between stations 5 and 6 at I-80 (see Figure 4.3) while an off-ramp resides between stations 2 and 3 (see Figure 4.4) at I-5. Other locations, such as the stretch between stations 7 and 8 , are not analyzed since there are multiple ramps as well as a lane reduction, and thus, we can only observe combined effects.

Figure 4.12 (a) illustrates the transition rates over four loop detector stations at I-80. It is notable that the median magnitude of transition rates increases from stations 5 to 6 (in the direction of kinematic wave) where an on-ramp is located. This trend is consistent in individual lanes as shown in Table 4.12. The table also presents the $p$-values from K-W and ANOVA tests, confirming the statistical significance in the increases in transition rate in all lanes. To the contrary, the magnitude of transition rate decreases at I-5 from stations 2 to 3 where an off-ramp resides. Thus, the off-ramp appears to have an opposite effect compared to the on-ramp. However, this decrease turns out to be statistically insignificant, which may be attributable to the small sample size (11 queues) or small offramp flow. 

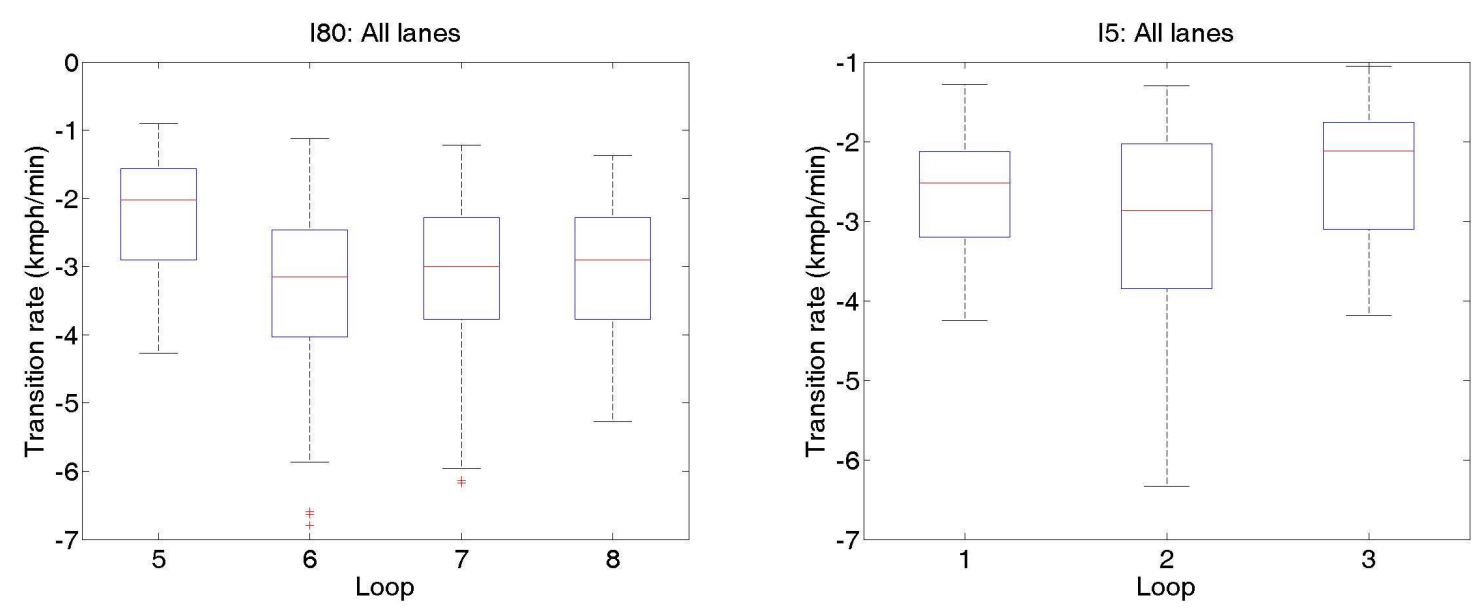

Figure 4.12: Median transition rates at onsets of queues over loop detector stations

(a) I-80; (b) I-5.

Table 4.12: Median Transition Rates at Loop Stations 5 and 6, I-80 (Onset)

\begin{tabular}{ccccc}
\hline & \multicolumn{2}{c}{ Median transition rate $(\mathrm{kmph} / \mathrm{min})$} & \multicolumn{2}{c}{ Statistical difference } \\
\cline { 2 - 5 } Lane & Loop 5 & Loop 6 & K-W Test & One-way ANOVA \\
\hline 2 & -2.67 & -4.33 & 0.00 & 0.00 \\
3 & -2.02 & -2.77 & 0.00 & 0.00 \\
4 & -1.97 & -2.67 & 0.01 & 0.01 \\
5 & -1.72 & -3.06 & 0.00 & 0.00 \\
\hline
\end{tabular}

Table 4.13: Median Transition Rates at Loop Stations 2 and 3, I-5 (Onset)

\begin{tabular}{ccccc}
\hline & \multicolumn{2}{c}{ Median transition rate $(\mathrm{kmph} / \mathrm{min})$} & \multicolumn{2}{c}{ Statistical difference } \\
\cline { 2 - 5 } Lane & Loop 2 & Loop 3 & K-W Test & One-way ANOVA \\
\hline 1 & -3.60 & -1.83 & 0.01 & 0.01 \\
2 & -2.60 & -2.47 & 0.53 & 0.37 \\
3 & -2.52 & -2.25 & 0.85 & 0.52 \\
\hline
\end{tabular}

\subsubsection{Clearance of Queue}

In this section, the transition characteristics are studied as the congestion clears (i.e., as the queue starts dissipating). Table 4.14 shows the median transition durations and rates for each lane at each site. The durations range from 10 to 30 minutes, marking transition rates of 2.0 to 6.2 $\mathrm{kmph} / \mathrm{min}$ depending on the site. The results for the queue dissipations show similar trends as those for congestion onsets, such that:

- The durations at M4 and A4 are smaller than those at I-80 and I-5 by comparison.

- M4 displays the largest magnitude of transition rates while I-5 displays the lowest transition rates. 
Table 4.14: Median Transition Durations and Rates at Four Study Sites (Clearance)

\begin{tabular}{ccccccccc}
\hline & \multicolumn{3}{c}{ Transition duration (mins) } & \multicolumn{4}{c}{ Transition rate $(\mathrm{kmph} / \mathrm{min})$} \\
\cline { 2 - 9 } & $\mathrm{M} 4$ & $\mathrm{~A} 4$ & $\mathrm{I}-80$ & $\mathrm{I}-5$ & $\mathrm{M} 4$ & $\mathrm{~A} 4$ & $\mathrm{I}-80$ & $\mathrm{I}-5$ \\
\hline Lane 1 & 13.0 & 11.0 & - & 25.0 & 5.9 & 5.8 & - & 2.3 \\
Lane 2 & 14.5 & 10.0 & 20.3 & 25.0 & 6.2 & 5.1 & 3.4 & 2.5 \\
Lane 3 & 16.5 & 10.0 & 21.0 & 30.0 & 6.0 & 4.8 & 3.0 & 2.0 \\
Lane 4 & - & - & 20.5 & - & - & - & 2.8 & - \\
Lane 5 & - & - & 19.5 & - & - & - & 2.8 & - \\
\hline
\end{tabular}

\subsubsection{Correlation Coefficients}

The correlation coefficients for queue recovery were found to be similar to those for queue onsets. The $p$-values for recovery at the four study sites are summarized in Tables $4.15-4.18$, with the shaded $p$-values indicating the correlations of significant pairs. Speed-related variables are correlated with one another as well as with Lane and Loop, which is consistent with the results for queue onsets.

Table 4.15: Correlation Coefficients of Explanatory Variables: M4 (Clearance)

\begin{tabular}{ccccccccccc}
\hline & \multicolumn{4}{c}{ Correlation Coefficient } & \multicolumn{5}{c}{ p-value } \\
\cline { 2 - 12 } & Lane & Loop & $\boldsymbol{v}_{\boldsymbol{i}}$ & $\boldsymbol{v}_{\boldsymbol{e}}$ & $\Delta \boldsymbol{v}$ & Lane & Loop & $\boldsymbol{v}_{\boldsymbol{i}}$ & $\boldsymbol{v}_{\boldsymbol{e}}$ & $\Delta \boldsymbol{v}$ \\
\hline Lane & - & 0.01 & 0.32 & 0.85 & 0.74 & - & 0.84 & 0.00 & 0.00 & 0.00 \\
Loop & 0.01 & - & 0.12 & 0.09 & 0.03 & 0.84 & - & 0.05 & 0.11 & 0.64 \\
$\boldsymbol{v}_{\boldsymbol{i}}$ & 0.32 & 0.12 & - & 0.38 & -0.03 & 0.00 & 0.05 & - & 0.00 & 0.61 \\
$\boldsymbol{v}_{\boldsymbol{e}}$ & 0.85 & 0.09 & 0.38 & - & 0.80 & 0.00 & 0.11 & 0.00 & - & 0.00 \\
$\Delta \boldsymbol{v}$ & 0.74 & 0.03 & -0.03 & 0.80 & - & 0.00 & 0.64 & 0.61 & 0.00 & - \\
\hline
\end{tabular}

Table 4.16: Correlation Coefficients of Explanatory Variables: A4 (Clearance)

\begin{tabular}{ccccccccccc}
\hline & \multicolumn{4}{c}{ Correlation Coefficient } & \multicolumn{5}{c}{ p-value } \\
\cline { 2 - 11 } & Lane & Loop & $\boldsymbol{v}_{\boldsymbol{i}}$ & $\boldsymbol{v}_{\boldsymbol{e}}$ & $\Delta \boldsymbol{v}$ & Lane & Loop & $\boldsymbol{v}_{\boldsymbol{i}}$ & $\boldsymbol{v}_{\boldsymbol{e}}$ & $\Delta \boldsymbol{v}$ \\
\hline Lane & - & 0.00 & -0.17 & -0.80 & -0.58 & - & 1.00 & 0.04 & 0.00 & 0.00 \\
Loop & 0.00 & - & -0.17 & 0.02 & 0.14 & 1.00 & - & 0.04 & 0.83 & 0.09 \\
$\boldsymbol{v}_{\boldsymbol{i}}$ & -0.17 & -0.17 & - & 0.22 & -0.53 & 0.04 & 0.04 & - & 0.01 & 0.00 \\
$\boldsymbol{v}_{\boldsymbol{e}}$ & -0.80 & 0.02 & 0.22 & - & 0.71 & 0.00 & 0.83 & 0.01 & - & 0.00 \\
$\Delta \boldsymbol{v}$ & -0.58 & 0.14 & -0.53 & 0.71 & - & 0.00 & 0.09 & 0.00 & 0.00 & - \\
\hline
\end{tabular}

Table 4.17: Correlation Coefficients of Explanatory Variables: I-80 (Clearance)

\begin{tabular}{ccccccccccc}
\hline & \multicolumn{4}{c}{ Correlation Coefficient } & \multicolumn{5}{c}{ p-value } \\
\cline { 2 - 12 } & Lane & Loop & $\boldsymbol{v}_{\boldsymbol{i}}$ & $\boldsymbol{v}_{\boldsymbol{e}}$ & $\Delta \boldsymbol{v}$ & Lane & Loop & $\boldsymbol{v}_{\boldsymbol{i}}$ & $\boldsymbol{v}_{\boldsymbol{e}}$ & $\Delta \boldsymbol{v}$ \\
\hline Lane & 1.00 & 0.00 & -0.08 & -0.63 & -0.15 & - & 1.00 & 0.09 & 0.00 & 0.00 \\
Loop & 0.00 & 1.00 & -0.56 & 0.16 & 0.59 & 1.00 & - & 0.00 & 0.00 & 0.00 \\
$\boldsymbol{v}_{\boldsymbol{i}}$ & -0.08 & -0.56 & 1.00 & 0.11 & -0.68 & 0.09 & 0.00 & - & 0.02 & 0.00 \\
\hline
\end{tabular}




\begin{tabular}{ccccccccccc}
\hline $\boldsymbol{v}_{\boldsymbol{e}}$ & -0.63 & 0.16 & 0.11 & 1.00 & 0.47 & 0.00 & 0.00 & 0.02 & - & 0.00 \\
$\Delta \boldsymbol{v}$ & -0.15 & 0.59 & -0.68 & 0.47 & 1.00 & 0.00 & 0.00 & 0.00 & 0.00 & - \\
\hline
\end{tabular}

Table 4.18: Correlation Coefficients of Explanatory Variables: I-5 (Clearance)

\begin{tabular}{ccccccccccc}
\hline & \multicolumn{4}{c}{ Correlation Coefficient } & \multicolumn{5}{c}{ p-value } \\
\cline { 2 - 12 } & Lane & Loop & $\boldsymbol{v}_{\boldsymbol{i}}$ & $\boldsymbol{v}_{\boldsymbol{e}}$ & $\Delta \boldsymbol{v}$ & Lane & Loop & $\boldsymbol{v}_{\boldsymbol{i}}$ & $\boldsymbol{v}_{\boldsymbol{e}}$ & $\Delta \boldsymbol{v}$ \\
\hline Lane & - & 0.00 & -0.19 & -0.45 & -0.07 & - & 1.00 & 0.05 & 0.00 & 0.48 \\
Loop & 0.00 & - & 0.30 & -0.40 & -0.47 & 1.00 & - & 0.00 & 0.00 & 0.00 \\
$\boldsymbol{v}_{\boldsymbol{i}}$ & -0.19 & 0.30 & - & 0.04 & -0.84 & 0.05 & 0.00 & - & 0.69 & 0.00 \\
$\boldsymbol{v}_{\boldsymbol{e}}$ & -0.45 & -0.40 & 0.04 & - & 0.50 & 0.00 & 0.00 & 0.69 & - & 0.00 \\
$\Delta \boldsymbol{v}$ & -0.07 & -0.47 & -0.84 & 0.50 & - & 0.48 & 0.00 & 0.00 & 0.00 & - \\
\hline
\end{tabular}

\subsubsection{Effect of Lane}

Tables 4.19-4.22 show the results ( $p$-values) from the K-W test and one-way ANOVA for the four study sites. At the 0.05 level, a $p$-value smaller than 0.05 indicates that at least one lane has a mean or median different from other lanes at that station. The results are slightly different from those for queue onsets, such that the differences in duration across lanes are statistically insignificant at all locations at all four sites. However, the results are rather mixed for transition rates. At A4, only station 7 exhibited statistical differences in lane during queue onsets while stations 5-7 exhibit differences in lane during queue dissipations. Moreover, all stations showed differences in lane at I-80 during onsets while only stations 6 and 8 report statistical differences across lanes. Nevertheless, lane-specific transition characteristics are observed at these sites in terms of initial speed and amount of speed increases during transition.

Table 4.19: Statistical Test Results for the Effect of Lane: M4 (Clearance)

\begin{tabular}{ccccc}
\hline & \multicolumn{2}{c}{ Transition duration } & \multicolumn{2}{c}{ Transition rate } \\
\cline { 2 - 5 } Loop Number & K-W Test & One-way ANOVA & K-W Test & One-way ANOVA \\
\hline 4 & 0.59 & 0.34 & 0.73 & 0.59 \\
5 & 0.07 & 0.07 & 0.37 & 0.44 \\
6 & 0.92 & 1.00 & 0.22 & 0.23 \\
7 & 0.64 & 0.62 & 0.43 & 0.42 \\
\hline
\end{tabular}

Table 4.20: Statistical Test Results for the Effect of Lane: A4 (Clearance)

\begin{tabular}{ccccc}
\hline & \multicolumn{2}{c}{ Transition duration } & \multicolumn{2}{c}{ Transition rate } \\
\cline { 2 - 5 } Loop Number & K-W Test & One-way ANOVA & K-W Test & One-way ANOVA \\
\hline 2 & 0.27 & 0.35 & 0.58 & 0.87 \\
3 & 0.89 & 0.96 & 0.19 & 0.11 \\
4 & 0.33 & 0.34 & 0.81 & 0.63 \\
5 & 0.40 & 0.58 & $\mathbf{0 . 0 3}$ & $\mathbf{0 . 0 2}$ \\
6 & 0.68 & 0.93 & $\mathbf{0 . 0 2}$ & $\mathbf{0 . 0 1}$ \\
7 & 0.97 & 0.76 & $\mathbf{0 . 0 1}$ & $\mathbf{0 . 0 1}$ \\
\hline
\end{tabular}

Table 4.21: Statistical Test Results for the Effect of Lane: I-80 (Clearance)

\begin{tabular}{ccccc}
\hline & \multicolumn{2}{c}{ Transition duration } & \multicolumn{2}{c}{ Transition rate } \\
\cline { 2 - 5 } Loop Number & K-W Test & One-way ANOVA & K-W Test & One-way ANOVA \\
\hline 5 & 0.07 & 0.08 & 0.92 & 0.86 \\
6 & 0.16 & 0.23 & $\mathbf{0 . 0 0}$ & $\mathbf{0 . 0 0}$
\end{tabular}


Table 4.22: Statistical Test Results for the Effect of Lane: I-5 (Clearance)

\begin{tabular}{ccccc}
\hline & \multicolumn{2}{c}{ Transition duration } & \multicolumn{2}{c}{ Transition rate } \\
\cline { 2 - 5 } Loop Number & K-W Test & One-way ANOVA & K-W Test & One-way ANOVA \\
\hline 1 & 0.25 & 0.38 & 0.17 & 0.23 \\
2 & 0.61 & 0.49 & 0.34 & 0.66 \\
3 & 0.55 & 0.48 & 0.42 & 0.60 \\
\hline
\end{tabular}

\subsubsection{Effect of Distance from a Bottleneck}

Similar to queue onsets, we perform K-W and ANOVA tests to assess statistical differences in transition durations and rates during queue clearance with respect to the loop station number (a proxy for the distance from the bottlenecks) at M4 and A4. The resulting $p$-values for M4 and A4 are shown in Tables 4.23 and 4.24. The results show that the $p$-values are well above 0.05 in all cases, indicating that transition structure does not change along characteristics in the absence of intervening ramps.

Table 4.23: Statistical Test Results for the Effect of Loop: M4 (Clearance)

\begin{tabular}{ccccc}
\hline & \multicolumn{2}{c}{ Transition duration } & \multicolumn{2}{c}{ Transition rate } \\
\cline { 2 - 5 } Lane Number & K-W Test & One-way ANOVA & K-W Test & One-way ANOVA \\
\hline 1 & 0.23 & 0.26 & 0.18 & 0.19 \\
2 & 0.83 & 0.92 & 0.85 & 0.54 \\
3 & 0.81 & 0.58 & 0.77 & 0.72 \\
\hline
\end{tabular}

Table 4.24: Statistical Test Results for the Effect of Loop: A4 (Clearance)

\begin{tabular}{ccccc}
\hline & \multicolumn{2}{c}{ Transition duration } & \multicolumn{2}{c}{ Transition rate } \\
\cline { 2 - 5 } Lane Number & K-W Test & One-way ANOVA & K-W Test & One-way ANOVA \\
\hline 1 & 0.59 & 0.75 & 0.16 & 0.24 \\
2 & 0.81 & 0.84 & 0.48 & 0.51 \\
3 & 0.45 & 0.52 & 0.20 & 0.17 \\
\hline
\end{tabular}

\subsubsection{Effects of Ramps}

The results for the effects of on- and off-ramps during queue clearance are consistent with those during queue onsets. Figure 4.13(a) illustrates the effect of the on-ramp on I80 based on the transition rates over the loop detector stations. It is notable that the median magnitude of transition rate is larger at station 6 than at station 5, indicating that the on-ramp inflow decreases the transition rate in the direction of (forward-moving) wave propagation. This is confirmed in Table 4.25, which shows $p$-values that are smaller than or close to 0.05 with the exception of lane 5 .

For the off-ramp located between stations 2 and 3 on I-5, the median transition rate is smaller at station 3 (see Figure 4.13(b)), indicating that the exiting flow increases the transition rate in the direction of wave propagation. However, as in the case for queue onsets, the statistical significance is not confirmed. 

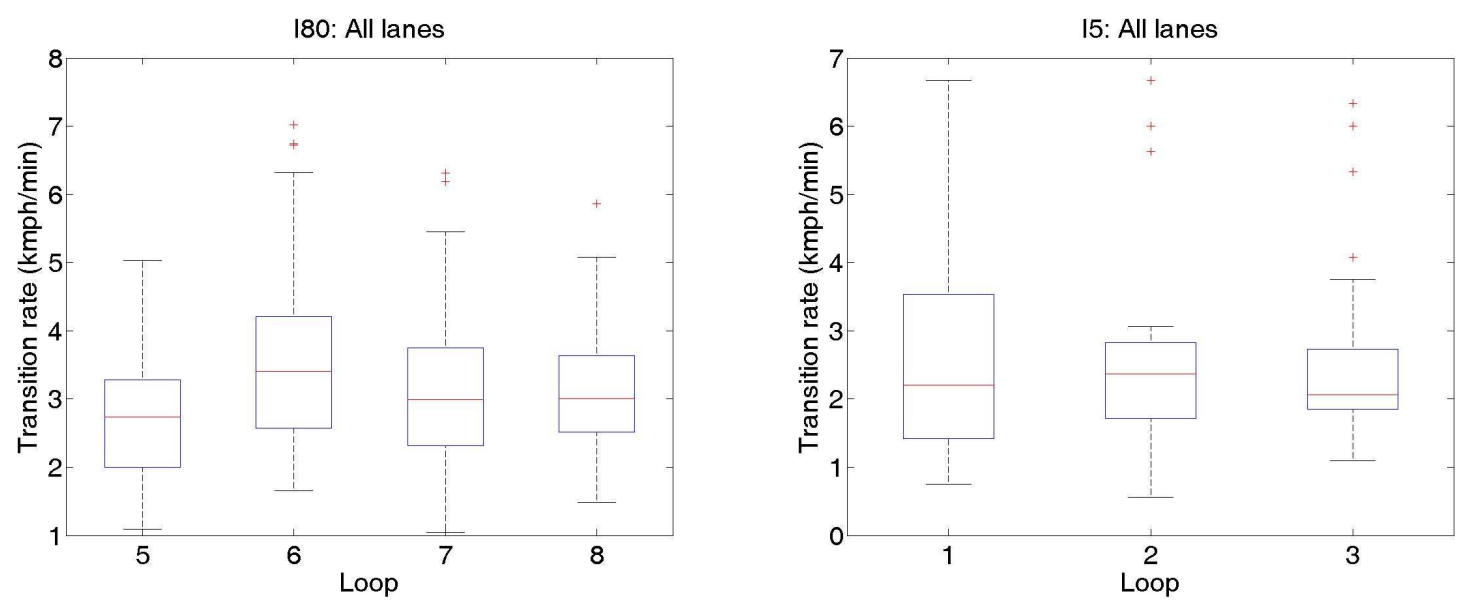

Figure 4.13: Median transition rates at onsets of queues over loop detector stations

(a) I-80 (b) I-5.

Table 4.25: Median Transition Rates at Loop Stations 5 and 6, I-80 (Clearance)

\begin{tabular}{ccccc}
\hline & \multicolumn{2}{c}{ Median transition rate $(\mathrm{kmph} / \mathrm{min})$} & \multicolumn{2}{c}{ Statistical difference } \\
\cline { 2 - 5 } Lane & Loop 5 & Loop 6 & K-W Test & One-way ANOVA \\
\hline 2 & 2.93 & 4.56 & 0.00 & 0.00 \\
3 & 2.74 & 3.20 & 0.02 & 0.01 \\
4 & 2.73 & 3.33 & 0.06 & 0.03 \\
5 & 2.57 & 2.89 & 0.14 & 0.10 \\
\hline
\end{tabular}

Table 4.26: Median Transition Rates at Loop Stations 2 and 3, I-5 (Clearance)

\begin{tabular}{ccccc}
\hline & \multicolumn{2}{c}{ Median transition rate $(\mathrm{kmph} / \mathrm{min})$} & \multicolumn{2}{c}{ Statistical difference } \\
\cline { 2 - 5 } Lane & Loop 2 & Loop 3 & K-W Test & One-way ANOVA \\
\hline 1 & 2.60 & 2.00 & 0.28 & 0.71 \\
2 & 2.65 & 1.94 & 0.37 & 0.58 \\
3 & 1.90 & 2.72 & 0.14 & 0.26 \\
\hline
\end{tabular}




\subsection{CONCLUDING REMARKS}

\subsection{SUMMARY OF FINDINGS}

This study has provided empirical observations on the characteristics of state transitions. Two types of transitions were analyzed; static transitions near a merge and dynamic transitions along kinematic waves during the onsets and dissipations of queues.

The transitions near a merge were analyzed by observing the spatial changes in traffic flow, density and space-mean speed over short, contiguous segments. The results showed that the transition in flow occurred within a distance of about 90 meters while the transitions in density and space-mean speed occurred over longer distances of about 120 meters and 180 meters, respectively. Finally, the spatial evolution of flow and density traced on a flow-density plane revealed that the flow increased in the vicinity of the on-ramp while density is nearly constant; then downstream of the on-ramp, flow remained nearly constant while the density gradually decreased and converged to the equilibrium.

The dynamic transitions were studied on several freeway segments with and without interchanges. Findings are summarized as follows:

- Transition durations observed from the four sites vary from 10 to 24 minutes during queue onsets while the durations ranged from 10 to 30 minutes during queue dissipations. At each site, formations and dissipations of queues displayed similar durations.

- Transition rates during queue onsets ranged from -7.6 to $-2.2 \mathrm{kmph} / \mathrm{min}$ while they ranged from 2.0 to $6.2 \mathrm{kmph} / \mathrm{min}$ during dissipations.

- Lane-specific features are observed in terms of initial speeds (just prior to transition) and change in speed during transition. However, transition durations and rates exhibited lanespecific behavior only at some sites.

- Transition durations and rates remained constant in the absence of freeway interchanges. Thus, the structure of transition does not change as a queue propagates. This finding was consistent for both queue onsets and dissipations.

- Transition rates tend to be larger upstream of an on-ramp as compared to a downstream location. This was observed during both queue onsets and dissipations. This indicates that, in the direction of kinematic wave, on-ramp inflow tends to increase the transition rate during a queue formation while it tends to decrease the rate during queue dissipation.

- The effect of an off-ramp was observed to be the opposite to that of an on-ramp, such that transition rates tend to be smaller upstream of an off-ramp. This implies that, in the direction of kinematic wave, off-ramp outflow tends to decrease the transition rate during a queue formation while it tends to increase the rate during queue dissipation. 
This study provides valuable insights as to how long (over time and space) a transition takes place and how transition structure changes by various geometric and traffic features. These findings are important in understanding the errors associated with the first-order kinematic wave model, which assumes instantaneous regime change. Thus, a better understanding of traffic transitions can help assess the need for more complex traffic flow models.

\subsection{RECOMMENDATIONS FOR FUTURE STUDY}

The following recommendations are suggested for future investigations:

- In this research, less than 30 queues were analyzed at each site. Thus, a larger sample of queues at a site is desirable to perform a more rigorous statistical analysis and modeling (regression) and to obtain more statistically significant results.

- More freeway sites should be analyzed in order to confirm the findings from this study and to explain the site-specific features of transition.

- This research addressed two types of transitions - transitions at a merge and at the tails of queues. Analyses of transitions near other types of freeway features (off-ramps, lanereduction, etc.) and at the heads of queues (active bottlenecks) are left for future research.

- Transition characteristics near a merge may vary depending on traffic conditions (free flow, semi-congested, congested, etc.). Hence, it is recommended that transitions in different regimes be compared (i.e., the change in length of transition with respect to traffic states can be investigated to identify specific differences).

- Though the findings suggest that inhomogeneous freeway features affect the transitions during queue onsets and dissipations, the mechanism governing this influence is unknown. 


\section{REFERENCES}

Beckmann, M., McGuire, C. B., and Winsten, C. B. 1955. Studies in the economics of transportation. RM-1488. Yale University Press.

Brackstone, M., and McDonald, M. 2000. "Car following: A historical review." Transportation Research F, 2(4), 181-196.

Carll, R. R., and Homburger, W. S. 1962. "Some characteristics of peak period traffic." Highway Research. Board Bulletin, 351, 33-49.

Cassidy, M. J. 1998. "Bivariate relations in nearly stationary highway traffic." Transportation Research B, 32(1), 49-59.

Cassidy, M. J., and Coifman, B. 1997. "Relation among average speed, flow and density and analogous relation between density and occupancy." Transportation Research Record, 1591, 1-6.

Cassidy, M. J., and Mauch, M. 2001. "An observed traffic pattern in long freeway queues." Transportation Research A, 35A, 149-162.

Del Castillo, J. M., and Benitez, F. G. 1995. "On functional form of the speed-density relationship - i: General theory, ii: Empirical investigation.” Transportation Research B, 29, 373 - 406.

Chiabaut, N., Buisson, C., and Leclercq, L. 2008a. "Fundamental diagram estimation through passing rate measurements in congestion." Submitted for publication.

Chandler, R. E., Herman, R., and Montroll, E. W. 1958. "Traffic dynamics: studies in car following." Operations Research, 6(2), 165-184.

Chung, K., and Cassidy, M. J. 2002. Testing Daganzo's behavioral theory for multi-lane freeway traffic. UCB-ITS-PWP-2002-09, University of California, Berkeley.

Cleveland, D. E., and Capelle, D. G. 1964. "Queuing theory approaches." An Introduction to Traffic Flow Theory, 49-96. Highway Research Board Special Report, 79.

Cremer, M., and May, A. D. 1985. An extended traffic model for freeway control. UCB-ITS-RR85-7, Institute of Transportation Studies, University of California, Berkeley, CA.

Daganzo, C. F. 1994. "The cell transmission model, part I: A simple dynamic representation of highway traffic consistent with the hydrodynamic theory." Transportation Research B, 28(2), 269-287. 
Daganzo, C. F. 1995. "The cell transmission model, part II: Network traffic." Transportation Research B, 29(2), 79-93.

Daganzo, C. F. 1997a. "A continuum theory of theory of traffic dynamics for freeways with special lanes." Transportation Research B, 31(2), 83-102.

Daganzo, C. F. 1997b. Fundamental of Transportation and Traffic Operations. Elsevier Science Ltd., 2nd edition. ISTB 0-08-042785-5.

Daganzo, C. F., and Laval, J. A. 2005. "On the numerical treatment of moving bottlenecks." Transportation Research B, 39, 31-46.

Daganzo, C. F., Cassidy, M. J., and Bertini, R. L. 1999. "Possible explanation of phase transitions in highway traffic." Transportation Research A, 33, 365-379.

Daganzo, C. F., Lin, W. H., and Del Castillo, J. M. 1997c. "A simple physical principle for the simulation of freeways with special lanes and priority vehicles." Transportation Research $B$, 31(2), 103-125.

Daganzo, C. F. 2002a. "A behavioral theory of multi-lane traffic flow. part I: Long homogeneous freeway sections." Transportation Research B, 36(2), 131-158.

Daganzo, C. F. 2002b. "A behavioral theory of multi-lane traffic flow. part II: Merges and the onset of congestion." Transportation Research B, 36(2), 159-169.

Edie, L. C., and Foote, R. S. 1958. "Traffic flow in tunnels." Highway Research Board Proceedings, 37, 334-344.

Evans, L., and Rothery, R. 1977. "Perceptual thresholds in car following - a recent comparison." Transportation Science, 11 (1), 60-72.

Gazis, D. C. 2002. "The origins of traffic theory." Operations Research, 50(1), 69-77.

Gazis, D. C., Herman, R., and Potts, R. 1959. "Car-following theory of steady-state traffic flow." Operations Research, 7 (4), 499-505.

Gazis, D. C., Herman, R., and Richard, W. 1961. "Nonlinear follow-the-leader models of traffic flow." Operations Research, 9(4), 545-67.

Gipps, P. 1981. "A behavioral car following model for computer simulation." Transportation Research B, 15(2), 105-111.

Helbing, D. 2001. "MASTER: macroscopic traffic simulation based on gas-kinetic, non-local traffic model." Transportation Research B, 35(2), 183-211. 
Helbing, D. 2001. "Traffic and related self-driven many-particle systems." Reviews of Modern Physics, 73, 1067-1141.

Herman, R., Montroll, E. W., Potts, R., and Rothery, R. 1959. "Traffic dynamics: Analysis of stability in car following." Operations Research, 7(1), 86-106.

Hranac, R., Margiotta, R., and Alexiadis, V. 2004. Next Generation Simulation (NGSIM) highlevel data plan, Report to FHWA, \#FHWA-HOP-06-011, FHWA, U.S. Department of Transportation.

Kerner, B., 2004. The Physics of Traffic: Empirical Freeway Pattern Features, Engineering Applications, and Theory. Springer, Understanding Complex Systems. ISBN: 3-540-20716-3.

Knopse, W., Santen, L., Schadschneider, A., and Schreckenberg, M. 2004. "An empirical test for cellular automation models of traffic flow." Physical Review E, 70(016115).

Kometani, E., and Sasaki, T. 1958. "On the stability of traffic flow." Journal of Operations Research, Japan, 2, 11-26.

Kumamoto, H., Nishi, K., Tenmoku, K., and Shimoura, H. 1995. "Rule based cognitive animation simulator for current lane and lane change drivers." Proceedings of the Second World Congress on ATT, Yokohama, Japan, 1746-1752.

Kunhe, R. 1984. "Macroscopic freeway model for dense traffic-stop-start waves and incident detection." Proceedings of the 9th International Symposium on Transportation and Traffic Theory (ISTTT19), 21-42.

Laval, J. A., and Daganzo, C. F. 2006. "Lane-changing in traffic streams." Transportation Research Part B, 40(3), 251-64.

Lebcque, J. 1993. "Les modeles macroscopiques de traffic.” Annales dePont, 67, 28-45.

Lebcque, J. 1996. "The Gudunov scheme and what it means for first order traffic flow models." Proceedings of the 13th International Symposium on Transportation and Traffic Theory (ISTTT), Pergamon, Oxford.

Lebcque, J. 2002. "A two phase extension of the LWR model based on the boundedness of traffic acceleration." Proceedings of the 15th International Symposium on Transportation and Traffic Theory, 697-718.

Lee, J., and Jones, J. H. 1967. "Traffic dynamics: visual angle car following models." Traffic Engineering and Control, 8, 348-350.

Leutzbach, W., and Wiedemann, R. 1986. "Development and applications of traffic simulation models at the Karlsruhe Institut fur Verkehrwesen." Traffic Engineering and Control, 27, 270278. 
Lighthill, M. J., and Whitham, G. B. 1955. "On kinematic waves. I. flood movement in long rivers." Proceedings of the Royal Society of London A, 229, 281-316.

Lighthill, M. J., and Whitham, G. B. 1955. "On kinematic waves. II. A theory of traffic flow on long crowded roads." Proceedings of the Royal Society of London A, 229, 317-345.

Mathewson, J. H., Trautman, D. L., and Gerlough, D. L. 1955. "Study of traffic flow by simulation." Proceedings of Highway Research Board, 34, 522-530.

Maerivoet, S., and Moor, B. D. 2005. "Transportation Planning and Traffic Flow Models". Internal Report, 05-155, ESAT-SCD (SISTA), K.U. Leuven, Leuven, Belgium.

Michaels, R. M. 1963. "Perceptual factors in car following." Proceedings of Second International Symposium on the Theory of Road Traffic Flow, Paris, OECD, 44-59.

Munoz, J. C., and Daganzo, C. F. 2003. "Structure of the transition zone behind freeway queues." Transportation Science, 37 (3), 312-29.

Nagel, K., and Schreckenberg, M. 1992. "A cellular automaton model for freeway." Journal De Physique, France, 2(12), 2221-2229.

Nagel, K. 1996. "Particle hopping models and traffic flow theory." Physical Review E, 53(5), 4655-4672.

Nagel, K., and Nelson, P. 2005. "A critical comparison of kinematic-wave model with observational data." Proceedings of the 16th International Symposium on Transportation and Traffic Theory (ISTTT16).

Navier, C. L. M. H. 1822. "Memoire sur les lois du mouvement des fluides". Mem. Acad. Sci. Inst. France, 6, 389-440.

Neumann, J. 1948. "The general and logical theory of automata." In L. A. Jeffress, editor, Cerebral mechanisms in Behavior, 1-41.

Newell, G. F. 1955. "Mathematical models for freely-flowing highway traffic." Journal of the Operations Research Society of America, 3(2), 176-86.

Newell, G. F. 1965. "Instability in dense highway traffic: A review." Proceedings of the Second International Symposium on the Theory of Traffic Flow, London, 73-83.

Newell, G. F. 1993. "A simplified theory of kinematic waves in highway traffic, part 1: General theory." Transportation Research B, 27(4), 281-287.

Newell, G. F. 1993. "A simplified theory of kinematic waves in highway traffic, part 2: Queuing at freeway bottlenecks." Transportation Research B, 27(4), 289-303. 
Newell, G. F. 1993. "A simplified theory of kinematic waves in highway traffic, part 3: Multidestination flows." Transportation Research B, 27(4), 305-313.

Newell, G. F. 2002. "A simplified car-following theory: a lower order model." Transportation Research B, 36(3), 195-205.

Papageorgiou, M., Blosseville, J. M., and Hadj-Salem, H. 1990. "Macroscopic modeling of traffic flow on the Boulevard Peripherique in Paris." Transportation Research B, 23B, 23-47.

Payne, H. J. 1971. "Models of freeway traffic and control." Mathematical Models of Public Systems (Simulation Council Proceedings), 1, 51-61.

Payne, H. J. 1978. FREFLO: A macroscopic simulation model of freeway traffic, Version 1 User's guide, ESSOR.

Pipes, L. A. 1953. "An operational analysis of traffic dynamics." Journal of Applied Physics, 24(3), 274-81.

Pipes, L. A. 1964. "Hydrodynamic approaches." An Introduction to Traffic Flow Theory, Highway Research Board Special Report, 79.

Reiter, U. 1994. "Empirical studies as basis for traffic flow models." Proceedings of the Second International Symposium on Highway Capacity, 2, 493-502.

Richards, P. I. 1956. "Shock waves on the highway." Operations Research, 4(1), 42-51.

Schadschneider, A. 2000. "Statistical physics of traffic flow." Physica A, 285, 101.

Schadschneider, A. 2002. "Traffic flow: A statistical physics point of view." Physica A, 313, 153-187.

Stokes, G. G. 1845. "On the theories of internal friction of fluids in motion." Trans. Camb. Philos. Soc., 8, 287-305.

Treiber, M., Hennecke, A., and Helbing, D. 1999. Explanation of Observed Features of SelfOrganization in Traffic Flow, E-print cond-mat/9901239.

Treiber, M., and Helbing, D. 2000. "Congested traffic states in empirical observations ad microscopic simulations.” Physical Review E, 62, 1805-1824.

Treiber, M., and Helbing, D. 2001. "Microsimulations of freeway traffic including control measures." Automatisierungstechnik, 49, 478-484.

Treiber, M., Kesting, A., and Helbing, D. 2005. "Delays, inaccuracies and anticipation in microscopic traffic models." Physica A, 360, 71-88. 
Yperman, I., Logghe, S., Tampere, C., and Immers, B. 2005. "The Multi-Commodity Link Transmission Model for Dynamic Network Loading." TRB 2006 Annual Meeting CD-ROM.

Wardrop, J. G. 1953. "Some theoretical aspects of road traffic research." Operations Research, 4 (4), 72-3.

Whitham, G. B. 1974. Linear and Nonlinear Waves, New York: John Wiley \& Son.

Wolf, F. 1999. “Cellular automata for traffic simulations.” Physics A, 263, 438-451.

Zhang, H. 1998. "A theory of non equilibrium traffic flow." Transportation Research B, 32(7), 485-498.

Zhang, H. 2001. "A finite difference approximation of a non-equilibrium traffic flow model." Transportation Research B, 34(4), 337-365.

Zhang, H. 2003. "Anisotropic property revisited - does it hold in multi-lane traffic?" Transportation Research B, 37(6), 561-577. 


\section{APPENDICES}




\section{APPENDIX A: OTHER SITES NOT INCLUDED IN THE REPORT}

\section{SITE: I-71 SB, OH, U.S.A.}

\section{Site description:}

Figure B-1 shows a schematic of southbound Interstate 71 near Columbus, Ohio. A major recurrent bottleneck (as labeled in the figure) activates during afternoon rush periods, and the resulting queues fill the general-purpose lanes for several hours. There are seven loop detector stations throughout the stretch, as shown in the figure. The distances between loop detector stations are different, from 500 meters to two kilometers, and there are many on- and off-ramps during this long distance. Data from each detector consist of event-based speed. Speed contours and time-speed plots are drawn from raw speed data, while these data were aggregated at 30second sampling intervals for more efficient handling. This site is suitable to study the effect of merges and diverges on transition durations. Data from one sample day on July 20, 2007, were analyzed. Whether more sample days will be introduced in the near future depends on the results of this sample day.

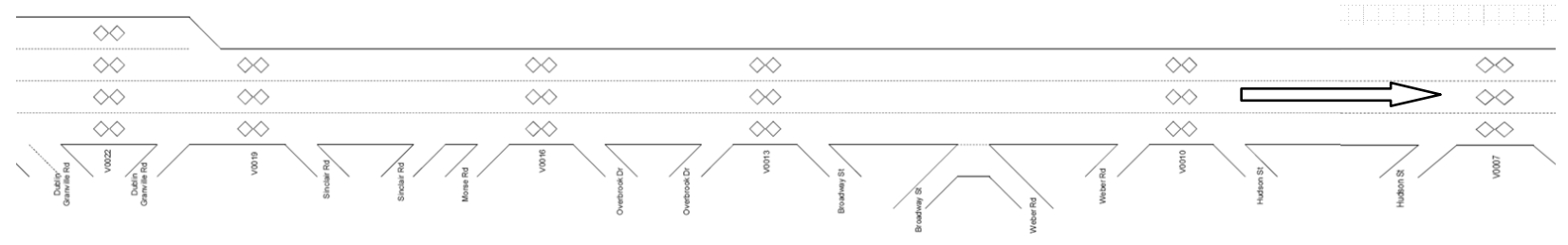

FIGURE B-1 Schematic of study site I-71 (SB), OH, U.S.A.

\section{Speed contours:}

Speed contours were constructed to assess general conditions on the study section. The all-lane speed contour of I-71 SB from 12-8 p.m.is presented in Figure A-2 as an example. As noted in the figure's legend, speed lower than $20 \mathrm{mph}$ is shaded in brown scale generated from the raw event-based speed. It shows heavy congestion for this facility from station 102 to station 10 and from 2-6 p.m. The figure illustrates several shock waves (brown zones in the figure) signaling a series of decelerations and accelerations as well as a few uncongested states during these four hours. 


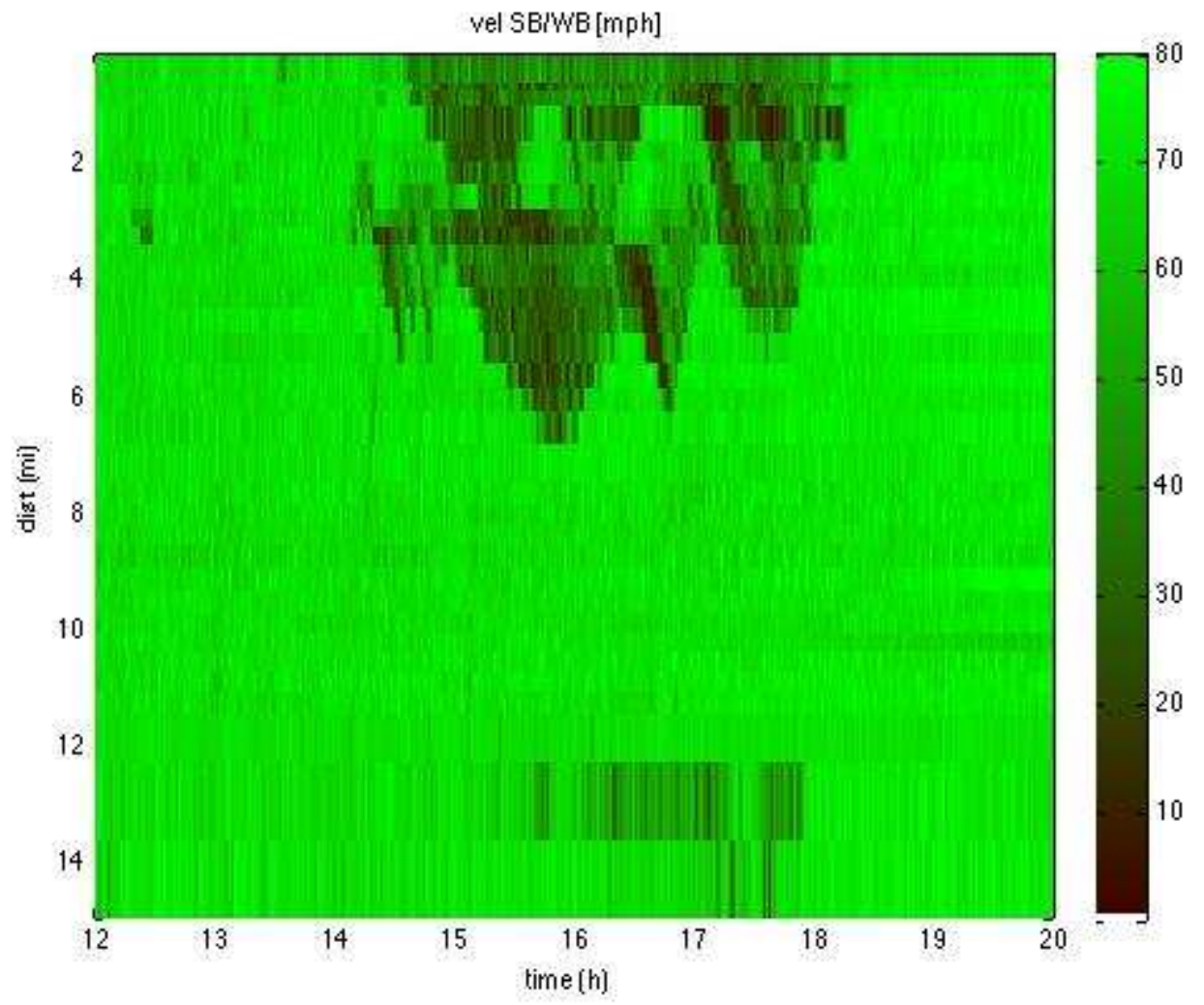

FIGURE A-2 Speed contour (all lanes) of I-71 SB from 12-8 p.m., July 20, 2007

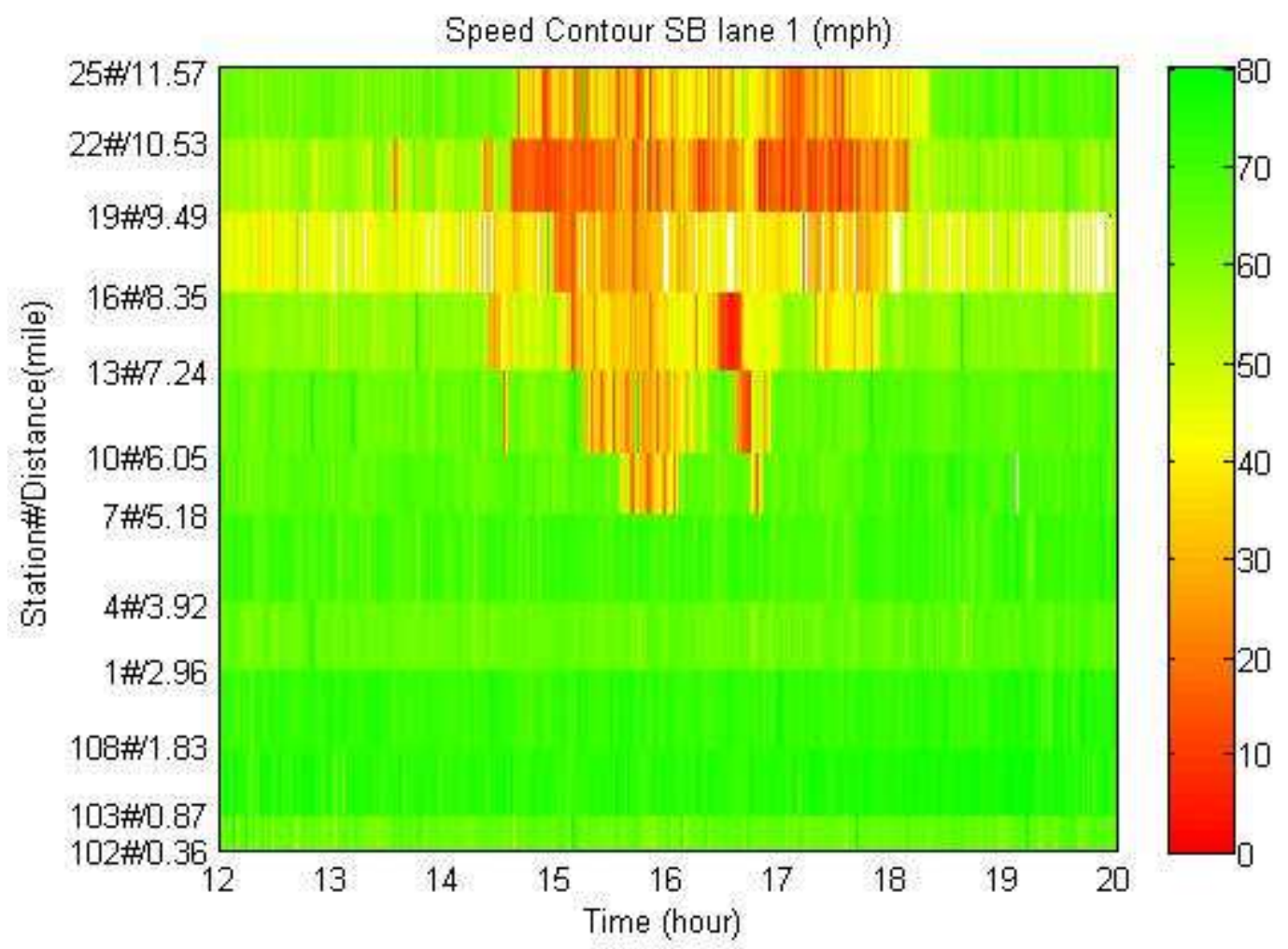

FIGURE A-3 Speed contour (lane 1) of I-71 SB from 12-8 p.m., July 20, 2007 


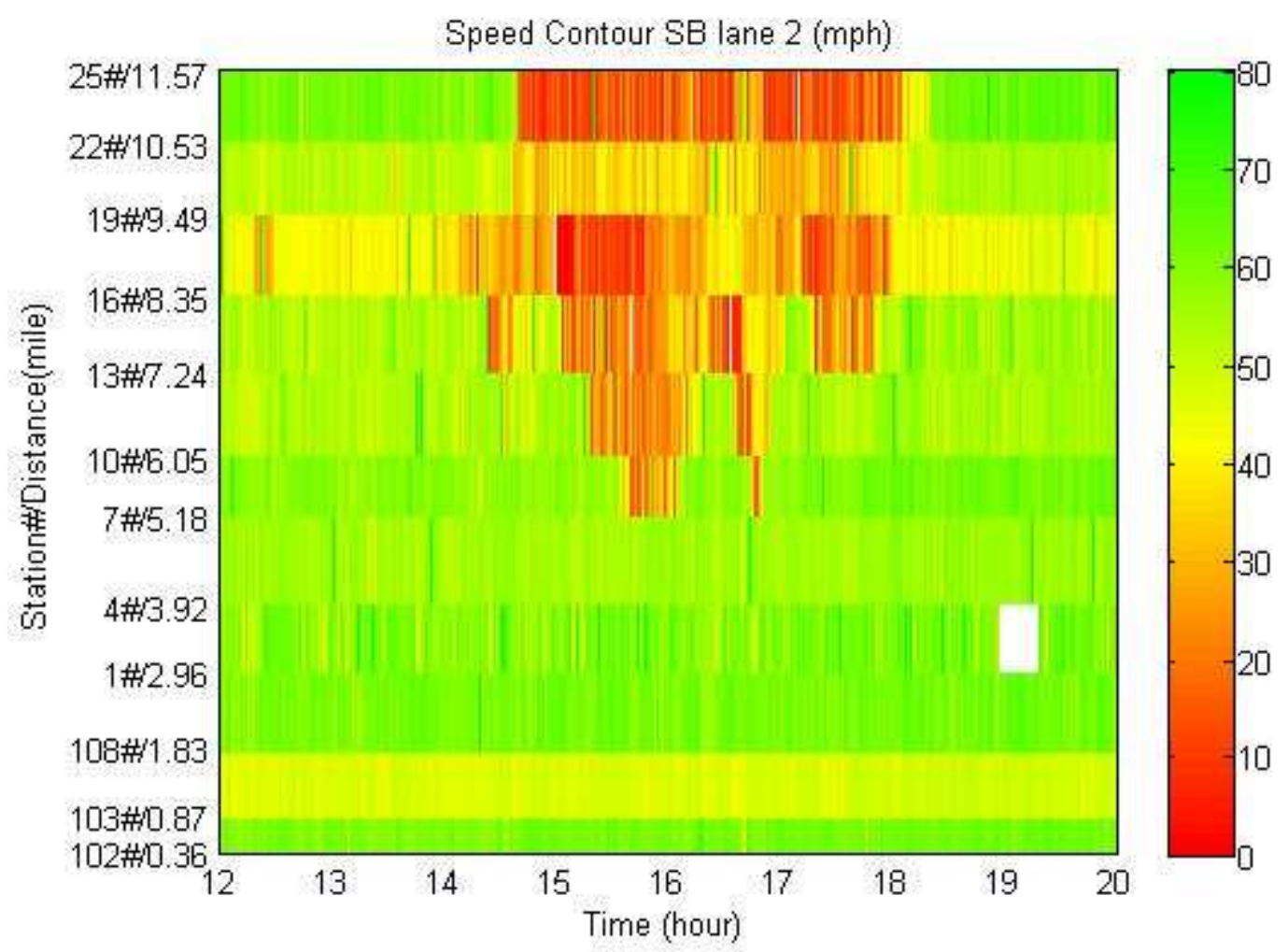

FIGURE A-4 Speed contour (lane 2) of I-71 SB from 12-8 p.m., July 20, 2007

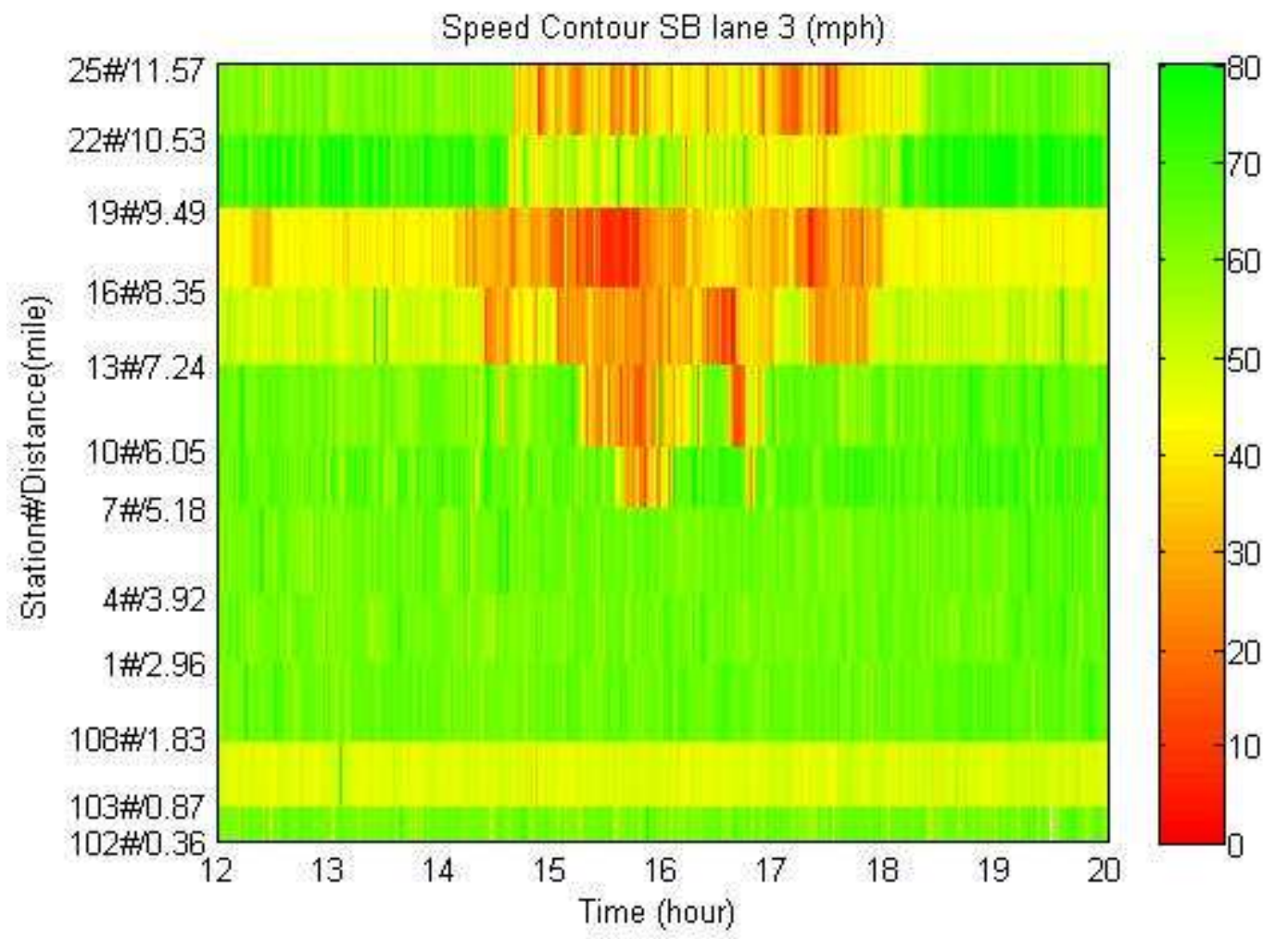

FIGURE A-5 Speed contour (lane 3) of I-71 SB from 12-8 p.m., July 20, 2007 


\section{Time series plots at each station at each lane:}
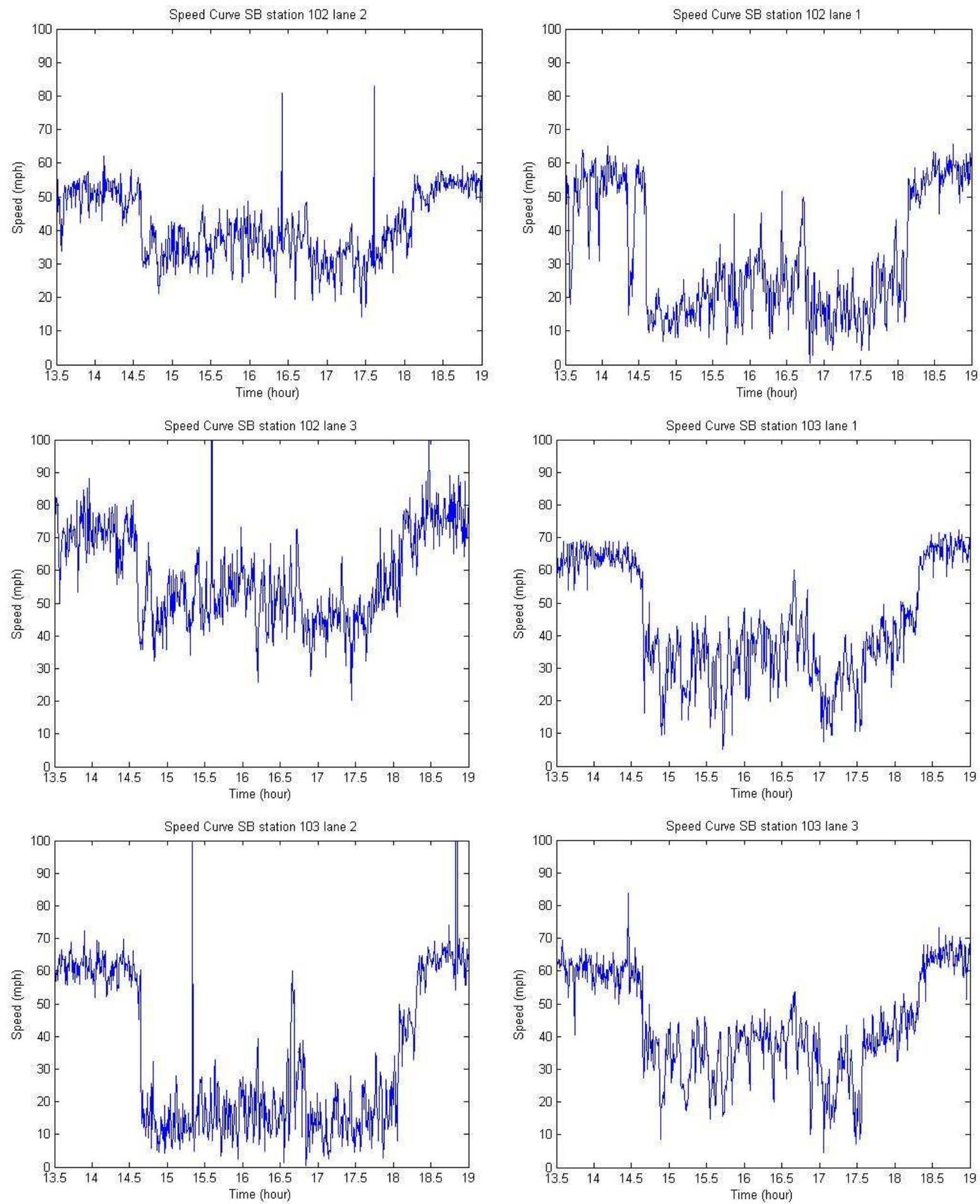

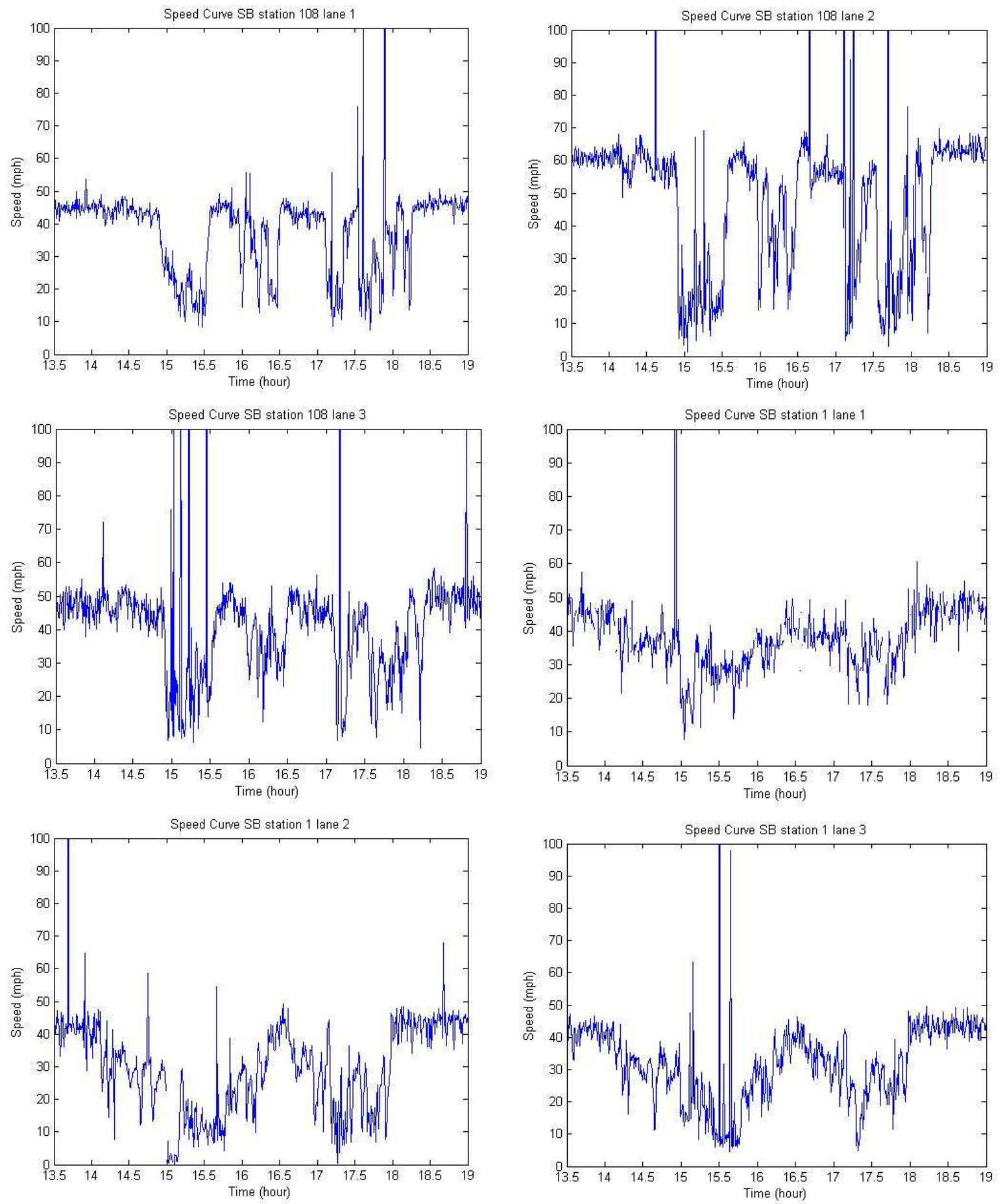

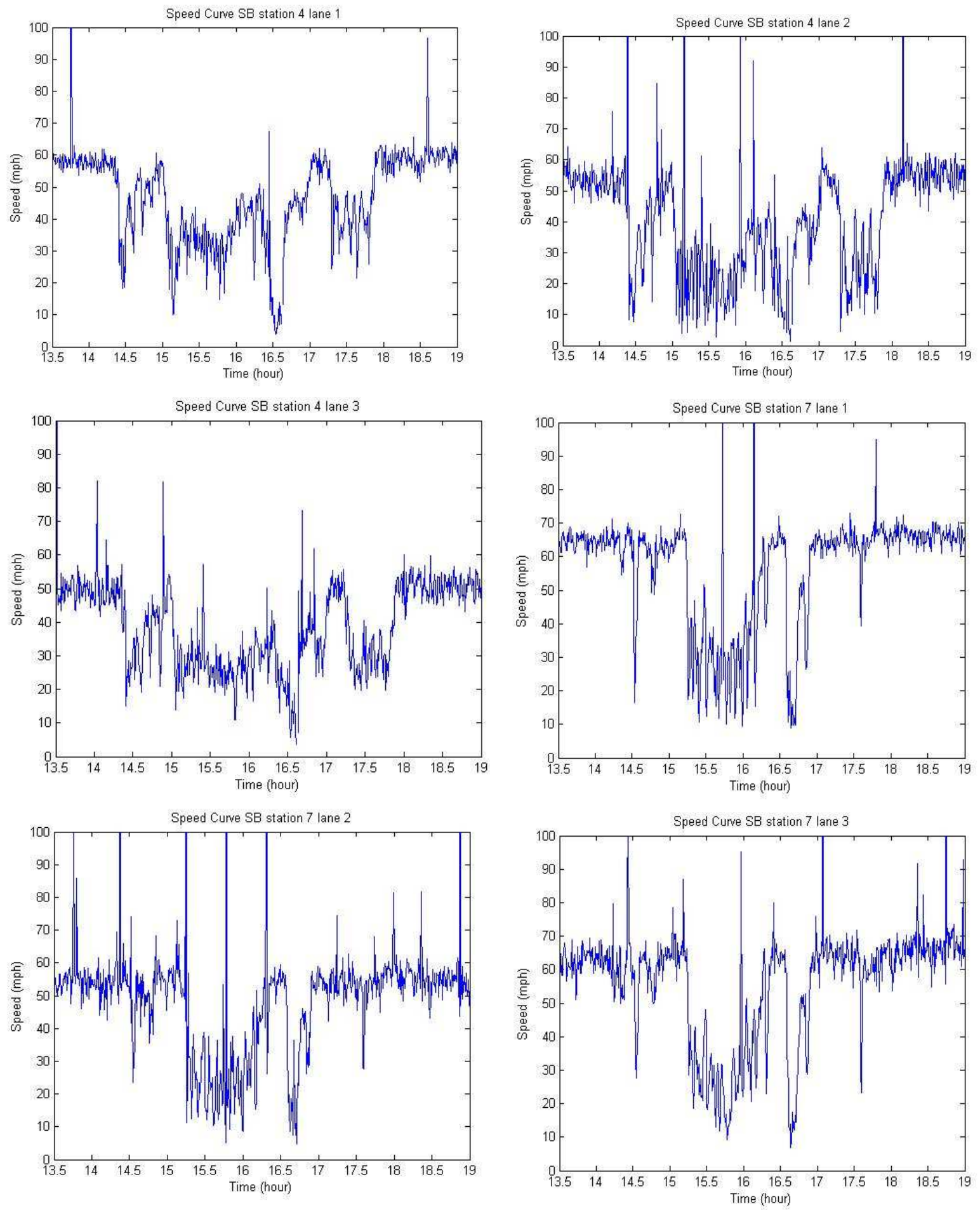

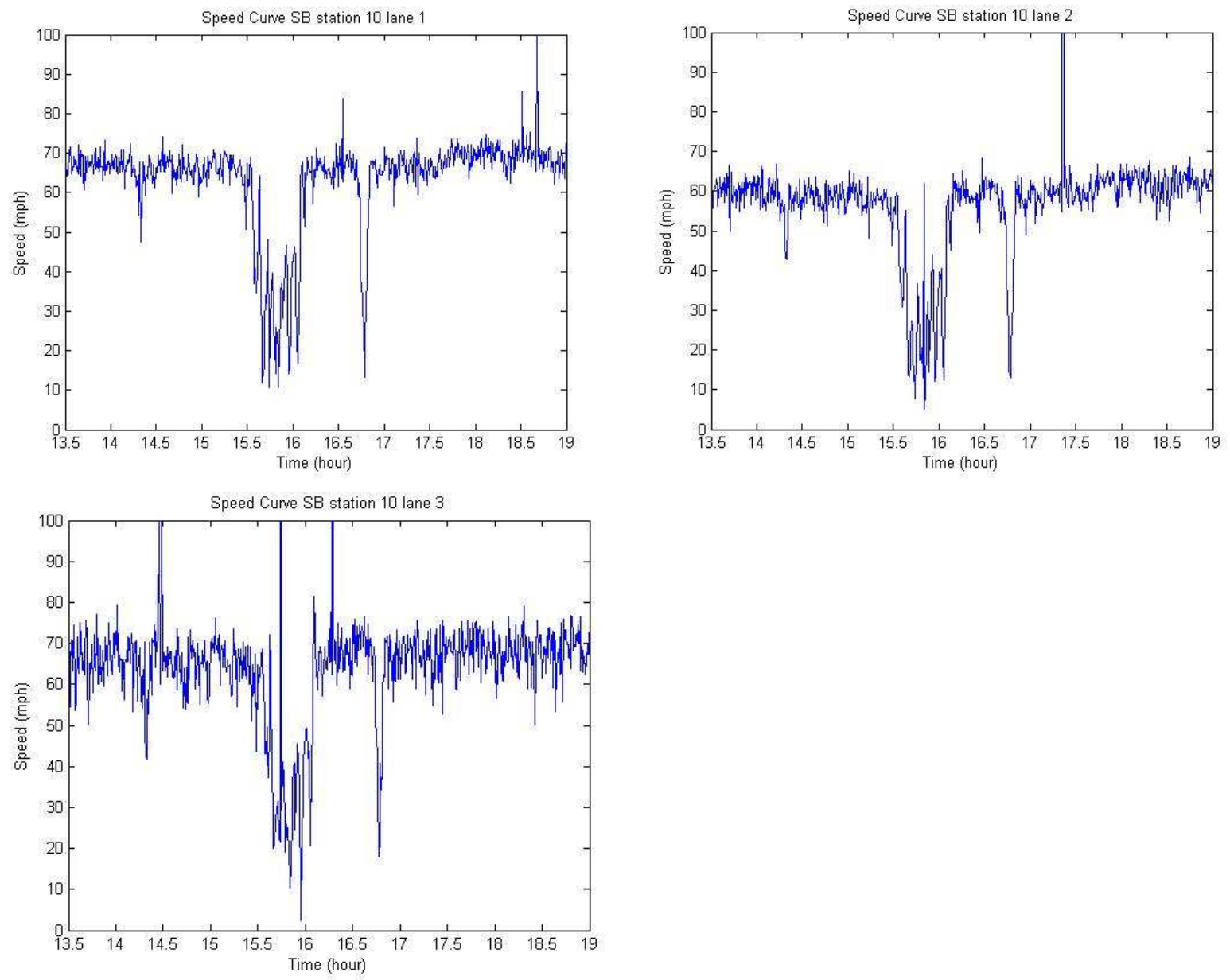

FIGURE A-6 Speed vs. Time (for each lane at each station) of I-71 SB from 1:30-7 p.m., July 20, 2007

\section{Results:}

In order to analyze the relationships between dependant variables (transition duration and transition rates) and explanatory variables (initial speed, end speed, speed reduction and wave speed, etc.), data are calculated according to the time-speed plots for each lane at each station. The results are shown below: 
Table A-1 dependant and explanatory variable data results of I-71 SB (Onset)

\begin{tabular}{|c|c|c|c|c|c|c|c|c|c|c|c|}
\hline Lane & Station & $\begin{array}{l}\text { Milepo } \\
\text { st }(\mathrm{km})\end{array}$ & $\begin{array}{c}\text { Start } \\
\text { time(h) }\end{array}$ & $\begin{array}{c}\text { End } \\
\text { time(h) }\end{array}$ & $\begin{array}{c}\text { Duratio } \\
\mathrm{n}(\mathrm{h})\end{array}$ & $\begin{array}{l}\text { Duration( } \\
\text { min.) }\end{array}$ & $\begin{array}{c}\text { Wave } \\
\text { speed } \\
(\mathrm{km} / \mathrm{h})\end{array}$ & $\begin{array}{c}\text { Start } \\
\text { speed(k } \\
\mathrm{m} / \mathrm{h})\end{array}$ & $\begin{array}{c}\text { End } \\
\text { speed(k } \\
\mathrm{m} / \mathrm{h})\end{array}$ & $\begin{array}{c}\Delta \text { Speed } \\
(\mathrm{km} / \mathrm{h})\end{array}$ & $\begin{array}{c}\text { Transitio } \\
\text { n rate } \\
(\mathrm{km} / \mathrm{h} / \mathrm{m} \\
\text { in. })\end{array}$ \\
\hline 1 & 102 & 0.59 & 14.56 & 14.61 & 0.05 & 3 & \multirow{7}{*}{-0.61} & 94.65 & 24.02 & -70.63 & -23.54 \\
\hline 1 & 103 & 1.40 & 14.64 & 14.66 & 0.02 & 1.2 & & 95.81 & 57.62 & -38.19 & -31.82 \\
\hline 1 & 108 & 2.94 & 14.9 & 14.97 & 0.07 & 4.2 & & 68.76 & 38.25 & -30.51 & -7.26 \\
\hline 1 & 1 & 4.76 & 14.96 & 15 & 0.04 & 2.4 & & 69.68 & 27.68 & -42.00 & -17.50 \\
\hline 1 & 4 & 6.31 & 14.35 & 14.41 & 0.06 & 3.6 & & 95.96 & 44.00 & -51.95 & -14.43 \\
\hline 1 & 7 & 8.34 & 15.2 & 15.26 & 0.06 & 3.6 & & 108.11 & 28.05 & -80.07 & -22.24 \\
\hline 1 & 10 & 9.74 & 15.53 & 15.59 & 0.06 & 3.6 & & 110.78 & 55.88 & -54.90 & -15.25 \\
\hline 2 & 102 & 0.59 & 14.58 & 14.62 & 0.04 & 2.4 & \multirow{7}{*}{-0.56} & 86.70 & 46.61 & -40.09 & -16.70 \\
\hline 2 & 103 & 1.40 & 14.64 & 14.67 & 0.03 & 1.8 & & 95.81 & 26.19 & -69.62 & -38.68 \\
\hline 2 & 108 & 2.94 & 14.88 & 14.93 & 0.05 & 3 & & 91.63 & 16.83 & -74.80 & -24.93 \\
\hline 2 & 1 & 4.76 & 14.11 & 14.14 & 0.03 & 1.8 & & 75.38 & 36.00 & -39.38 & -21.88 \\
\hline 2 & 4 & 6.31 & 14.35 & 14.41 & 0.06 & 3.6 & & 98.55 & 13.56 & -84.99 & -23.61 \\
\hline 2 & 7 & 8.34 & 15.16 & 15.32 & 0.16 & 9.6 & & 89.74 & 19.92 & -69.83 & -7.27 \\
\hline 2 & 10 & 9.74 & 15.63 & 15.68 & 0.05 & 3 & & 88.94 & 21.43 & -67.51 & -22.50 \\
\hline 3 & 102 & 0.59 & 14.57 & 14.64 & 0.07 & 4.2 & \multirow{7}{*}{-0.60} & 119.25 & 57.49 & -61.76 & -14.70 \\
\hline 3 & 103 & 1.40 & 14.63 & 14.66 & 0.03 & 1.8 & & 93.67 & 43.84 & -49.83 & -27.68 \\
\hline 3 & 108 & 2.94 & 14.89 & 14.95 & 0.06 & 3.6 & & 78.28 & 11.09 & -67.18 & -18.66 \\
\hline 3 & 1 & 4.76 & 14.2 & 14.23 & 0.03 & 1.8 & & 70.79 & 35.47 & -35.32 & -19.62 \\
\hline 3 & 4 & 6.31 & 14.35 & 14.41 & 0.06 & 3.6 & & 91.96 & 24.13 & -67.83 & -18.84 \\
\hline 3 & 7 & 8.34 & 15.23 & 15.26 & 0.03 & 1.8 & & 106.74 & 45.71 & -61.04 & -33.91 \\
\hline 3 & 10 & 9.74 & 15.55 & 15.59 & 0.04 & 2.4 & & 106.78 & 64.72 & -42.05 & -17.52 \\
\hline
\end{tabular}


Table A-2 dependant and explanatory variable data results of I-71 SB (Clearance)

\begin{tabular}{|c|c|c|c|c|c|c|c|c|c|c|c|}
\hline Lane & Station & $\begin{array}{c}\text { Milliag } \\
\text { e (km) }\end{array}$ & $\begin{array}{c}\text { Start } \\
\text { time(h) }\end{array}$ & $\begin{array}{c}\text { End } \\
\text { time(h) }\end{array}$ & $\begin{array}{c}\text { Duratio } \\
\mathrm{n}(\mathrm{h})\end{array}$ & $\begin{array}{l}\text { Duration( } \\
\text { min.) }\end{array}$ & $\begin{array}{c}\text { Wave } \\
\text { speed } \\
(\mathrm{km} / \mathrm{h})\end{array}$ & $\begin{array}{c}\text { Start } \\
\text { speed }(\mathrm{k} \\
\mathrm{m} / \mathrm{h})\end{array}$ & $\begin{array}{c}\text { End } \\
\text { speed(k } \\
\mathrm{m} / \mathrm{h})\end{array}$ & $\begin{array}{c}\Delta \text { Speed } \\
(\mathrm{km} / \mathrm{h})\end{array}$ & $\begin{array}{c}\text { Transiti } \\
\text { on rate } \\
(\mathrm{km} / \mathrm{h} / \\
\mathrm{min} .)\end{array}$ \\
\hline 1 & 102 & 0.59 & 18.1 & 18.16 & 0.06 & 3.6 & \multirow{7}{*}{0.29} & 20.38 & 88.92 & 68.54 & 19.04 \\
\hline 1 & 103 & 1.40 & 18.27 & 18.34 & 0.07 & 4.2 & & 64.72 & 103.51 & 38.78 & 9.23 \\
\hline 1 & 108 & 2.94 & 18.23 & 18.27 & 0.04 & 2.4 & & 21.77 & 72.55 & 50.78 & 21.16 \\
\hline 1 & 1 & 4.76 & 15.15 & 15.19 & 0.04 & 2.4 & & 19.80 & 59.88 & 40.07 & 16.70 \\
\hline 1 & 4 & 6.31 & 17.8 & 17.9 & 0.1 & 6 & & 41.49 & 98.39 & 56.90 & 9.48 \\
\hline 1 & 7 & 8.34 & 16.86 & 16.9 & 0.04 & 2.4 & & 44.18 & 101.83 & 57.65 & 24.02 \\
\hline 1 & 10 & 9.74 & 16.05 & 16.09 & 0.04 & 2.4 & & 26.84 & 107.05 & 80.21 & 33.42 \\
\hline 2 & 102 & 0.59 & 18.09 & 18.13 & 0.04 & 2.4 & \multirow{7}{*}{0.29} & 56.21 & 91.01 & 34.81 & 14.50 \\
\hline 2 & 103 & 1.40 & 18.27 & 18.34 & 0.07 & 4.2 & & 64.72 & 103.51 & 38.78 & 9.23 \\
\hline 2 & 108 & 2.94 & 18.22 & 18.27 & 0.05 & 3 & & 11.70 & 98.16 & 86.47 & 28.82 \\
\hline 2 & 1 & 4.76 & 17.92 & 17.98 & 0.06 & 3.6 & & 15.01 & 74.46 & 59.45 & 16.51 \\
\hline 2 & 4 & 6.31 & 17.81 & 17.91 & 0.1 & 6 & & 19.21 & 94.62 & 75.41 & 12.57 \\
\hline 2 & 7 & 8.34 & 16.71 & 16.79 & 0.08 & 4.8 & & 7.70 & 74.12 & 66.43 & 13.84 \\
\hline 2 & 10 & 9.74 & 16.05 & 16.1 & 0.05 & 3 & & 19.96 & 94.75 & 74.78 & 24.93 \\
\hline 3 & 102 & 0.59 & 17.44 & 17.48 & 0.04 & 2.4 & \multirow{7}{*}{0.42} & 32.80 & 77.04 & 44.24 & 18.43 \\
\hline 3 & 103 & 1.40 & 17.54 & 17.59 & 0.05 & 3 & & 14.04 & 73.17 & 59.13 & 19.71 \\
\hline 3 & 108 & 2.94 & 18.22 & 18.29 & 0.07 & 4.2 & & 7.16 & 81.08 & 73.92 & 17.60 \\
\hline 3 & 1 & 4.76 & 17.94 & 17.99 & 0.05 & 3 & & 32.33 & 72.18 & 39.85 & 13.28 \\
\hline 3 & 4 & 6.31 & 17.77 & 17.93 & 0.16 & 9.6 & & 31.70 & 86.01 & 54.31 & 5.66 \\
\hline 3 & 7 & 8.34 & 16.86 & 16.9 & 0.04 & 2.4 & & 46.29 & 97.81 & 51.52 & 21.47 \\
\hline 3 & 10 & 9.74 & 16.05 & 16.08 & 0.03 & 1.8 & & 33.07 & 110.96 & 77.89 & 43.27 \\
\hline
\end{tabular}

Once transition start and end times were identified, various explanatory variables were measured to identify factors that influenced transition durations along shock waves. Unlike the other four sites, there is only a one-day sample of data for this site. Thus, only the bivariate relations between transition duration and different lanes or stations are analyzed for this site. Statistical significance of influence of each explanatory variable will be studied in the future.

\section{Findings:}

The observed individual transition durations of SB I-71 on this sample day range from two to 10 minutes for both onset and clearance. The observed individual transition rates range from -7 to $39 \mathrm{~km} / \mathrm{h} / \mathrm{min}$ for onset and from 6 to $43 \mathrm{~km} / \mathrm{h} / \mathrm{min}$ for clearance.

\section{Effect of distance to the bottleneck on transition duration}

Figures A-7(a) and (b) show plots of average transition durations of all lanes (red circles) vs. stations located upstream of the bottleneck. The vertical lines crossing each circle represent the bounds of standard error. The average duration is 3.14 minutes for the onset and 3.66 minutes for 
clearance, and the standard error for both is less than one minute. It appears that the average durations do not change significantly longitudinally over space for both onset and clearance.

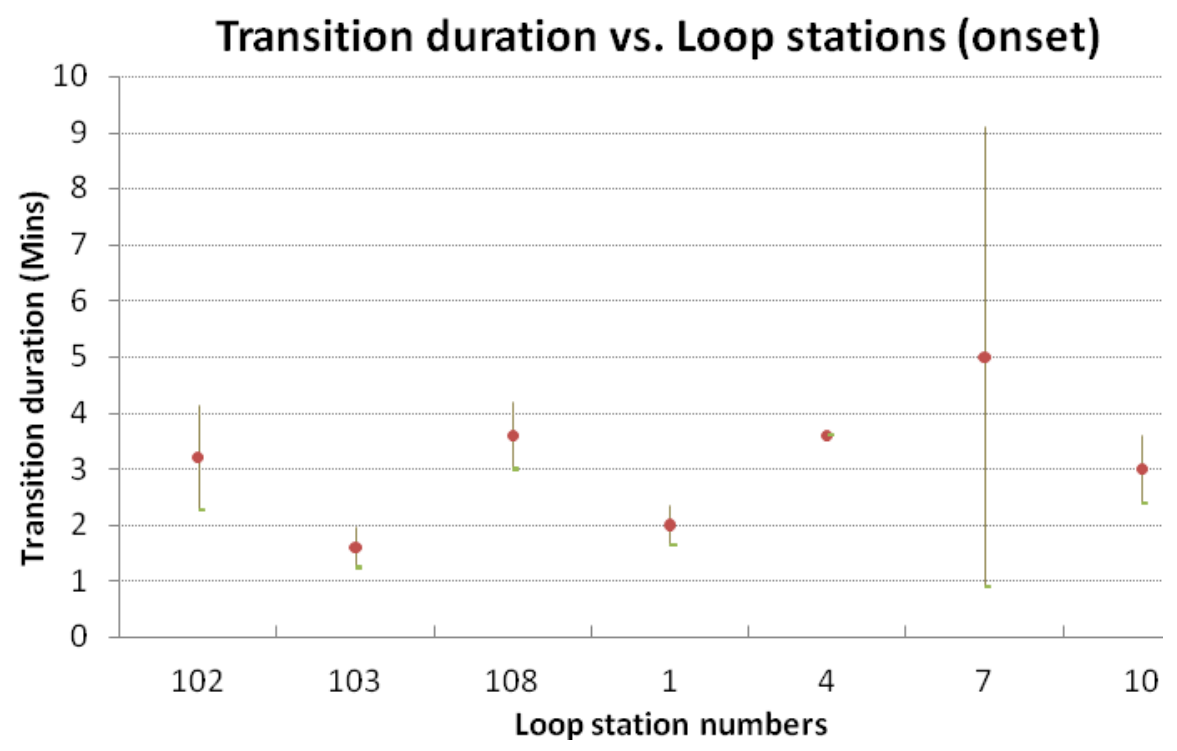

(a)

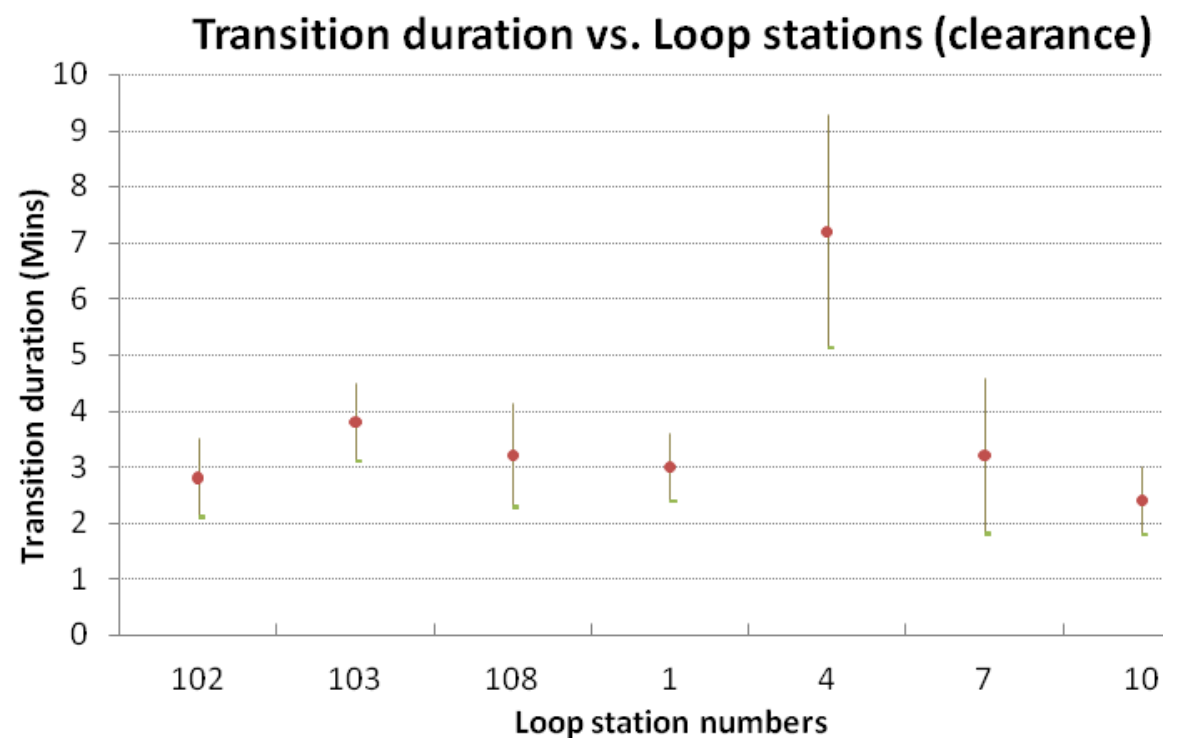

(b)

FIGURE A-7 Average transition duration (and standard error) vs. detector station for I-71 SB (a) all lanes onset; (b) all lanes clearance.

\section{Effect of lanes on transition duration}

In Figure A-8 (a) and (b), the average durations and their standard error bounds are presented by lane (with data from the seven stations combined). Figure A-8 (a) and (b) show that the average durations of both onset and clearance change little across lanes; only the average duration of onset is a little lower ( 0.5 minute) than that of clearance. 


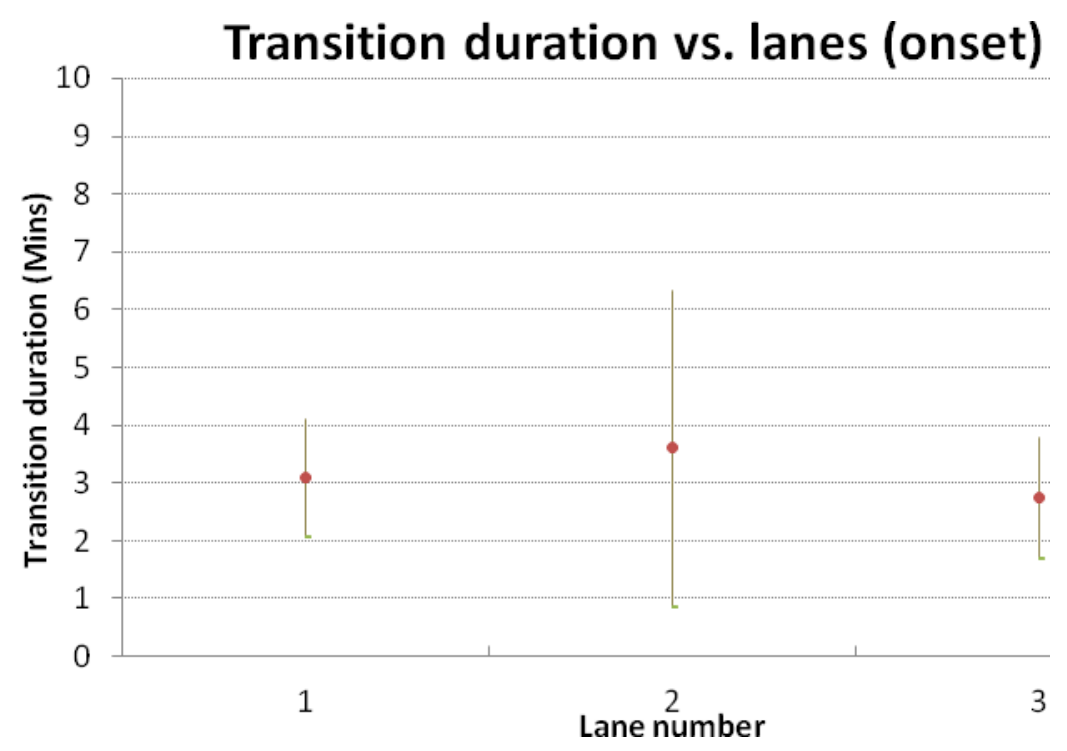

(a)

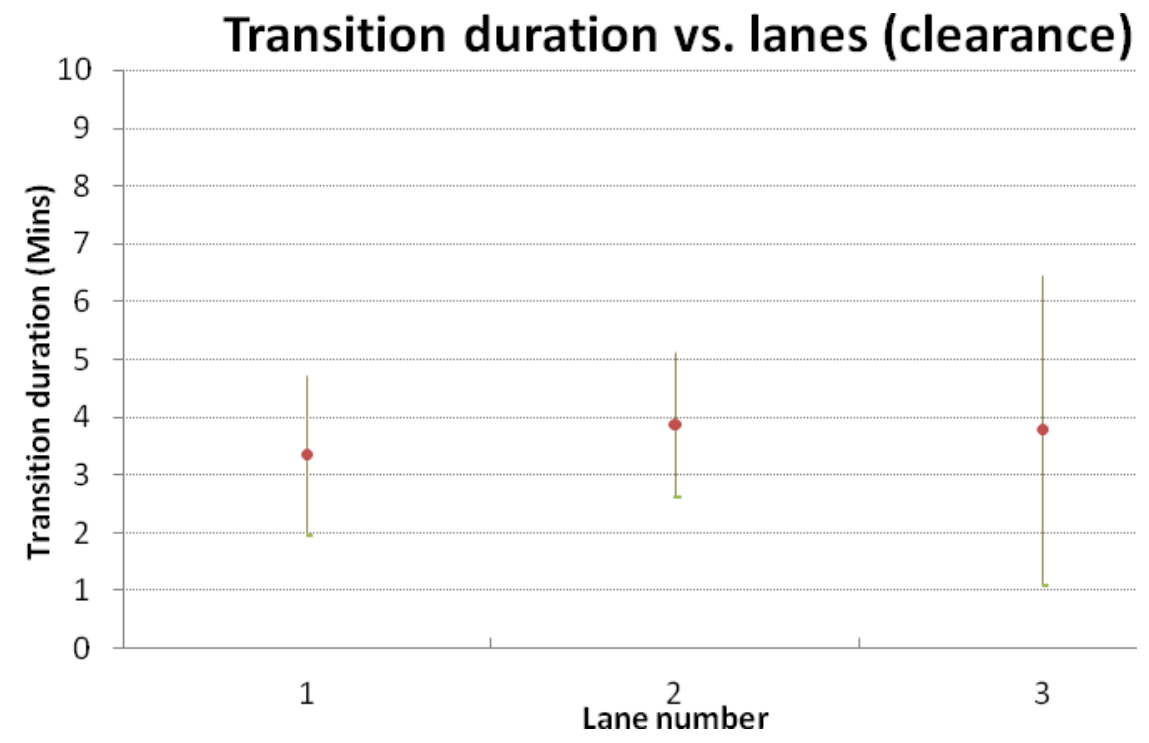

(b)

FIGURE A-8 Average transition duration (and standard error) vs. lane number for I-71 SB.

(a) All stations onset; (b) All stations clearance

\section{Effect of distance to the bottleneck on transition rate}

Figures A-9 (a) and (b) show plots of average transition rates of all lanes (red circles) vs. stations located upstream of the bottleneck. The vertical lines crossing each circle represent the bounds of standard error. The average rate is $-20.88 \mathrm{~km} / \mathrm{h} / \mathrm{min}$ for the onset with the standard deviation 6.14 $\mathrm{km} / \mathrm{h} / \mathrm{min}$, and the average rate is 18.71 minutes for clearance with the standard error 4.87 $\mathrm{km} / \mathrm{h} / \mathrm{min}$. It appears that the average rates do not change significantly longitudinally over space for both onset and clearance. 


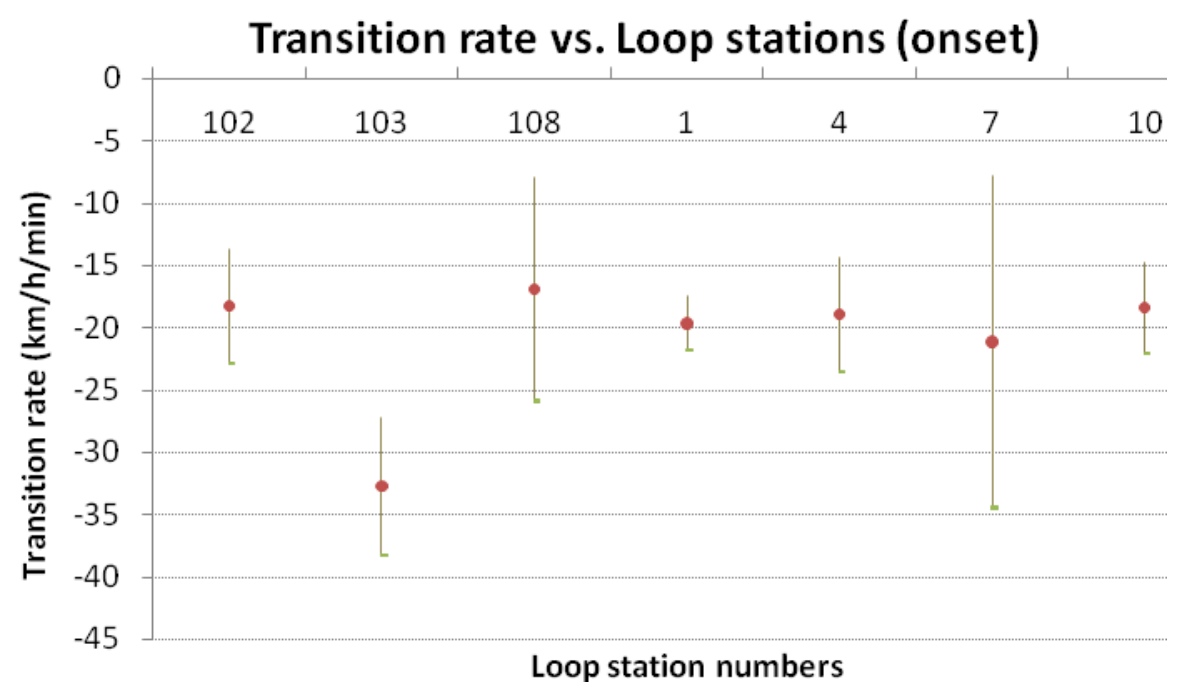

(a)

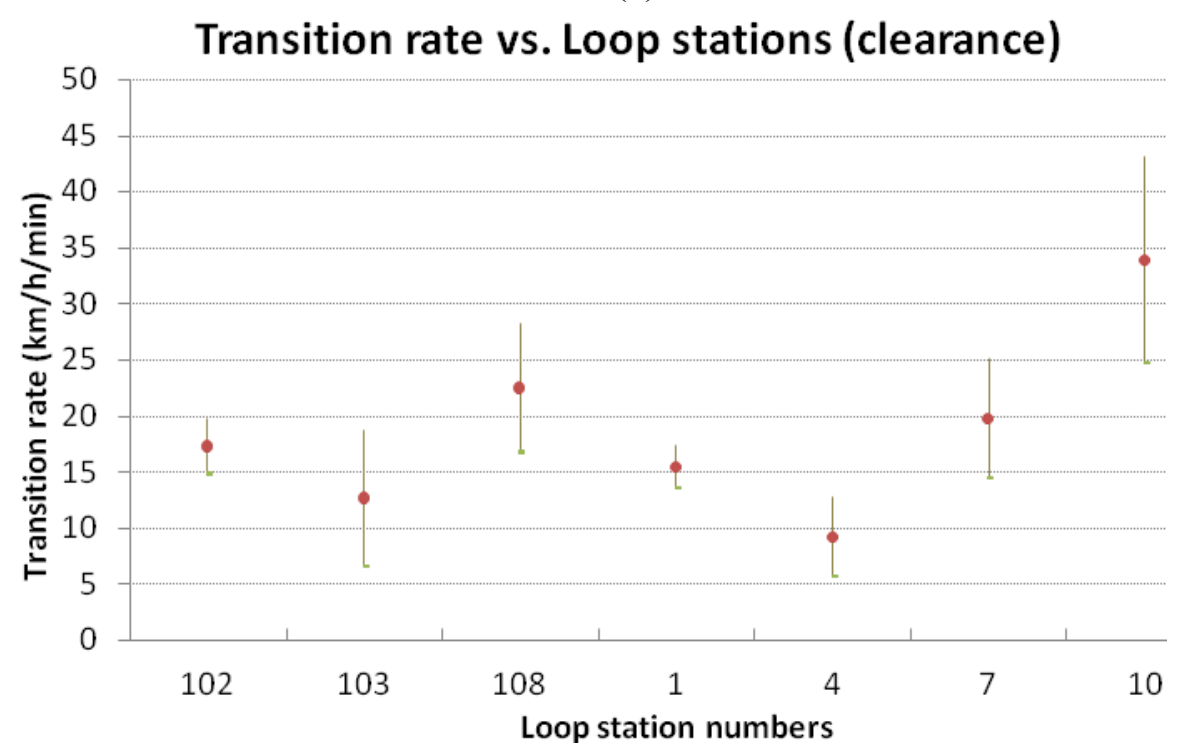

(b)

FIGURE A-9 Average transition rate (and standard error) vs. detector station for I-71 SB (a) all lanes onset; (b) all lanes clearance.

\section{Effect of lanes on transition rate}

In Figure A-10 (a) and (b), the average rates and their standard error bounds are presented by lane (with data from the seven stations combined). Figure A-10 (a) and (b) show that the average rates of both onset and clearance change little across lanes; only the average rate of onset is a little longer $(2.1 \mathrm{~km} / \mathrm{h} / \mathrm{min}$ in obsolete value $)$ than that of clearance. 


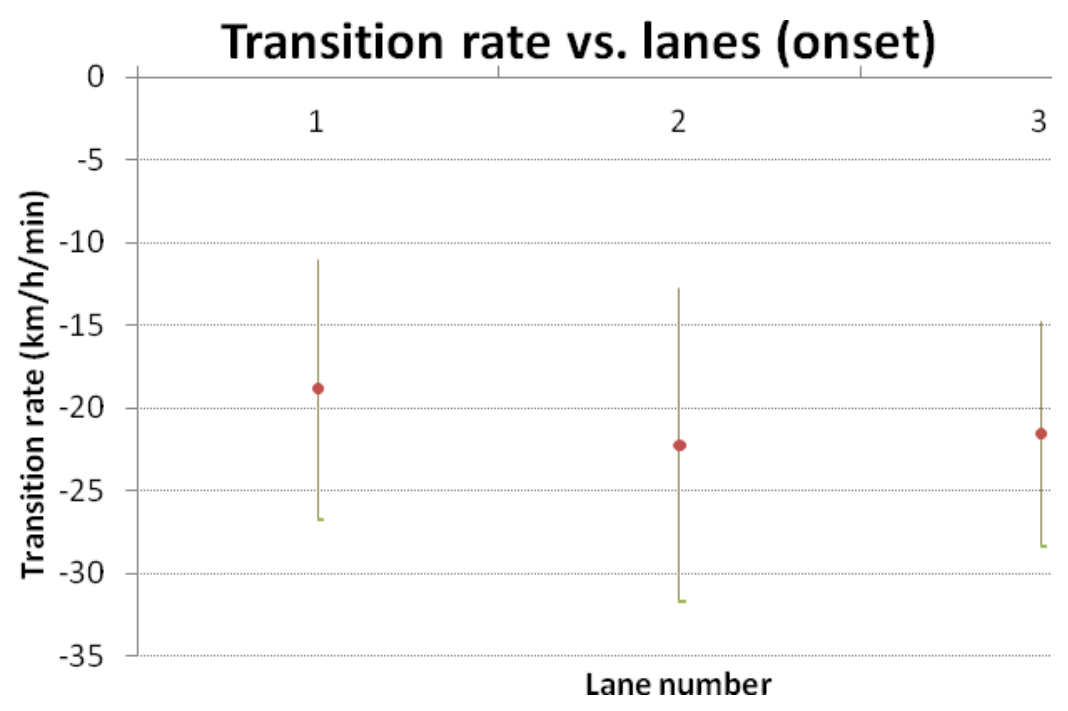

(a)

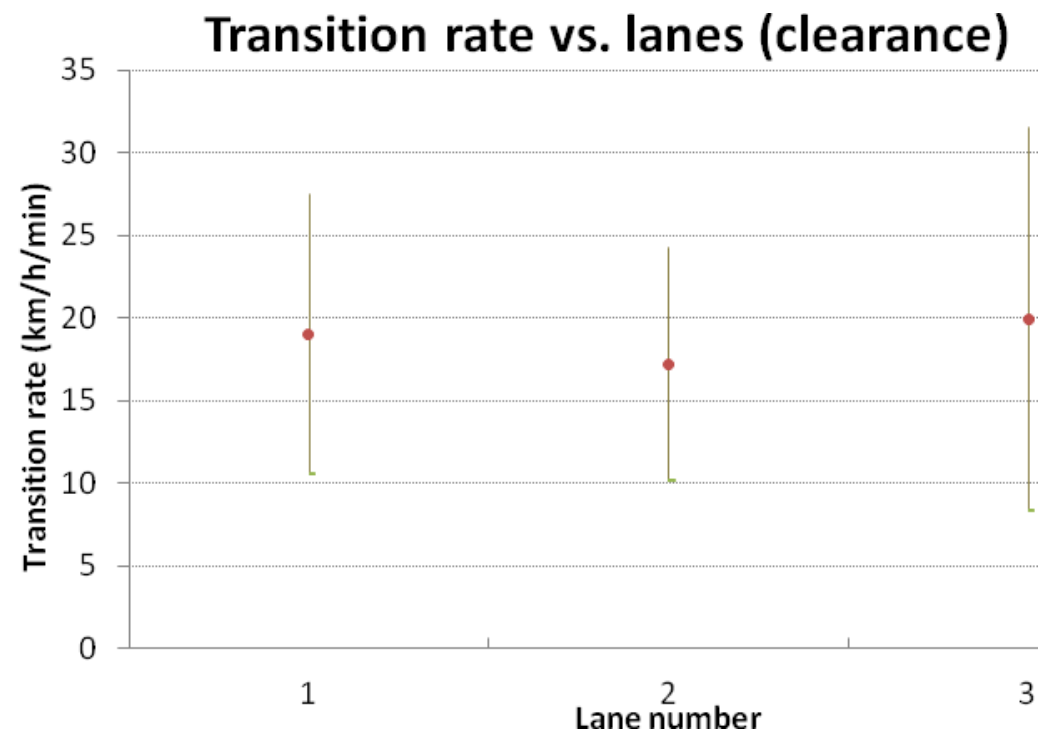

(b)

FIGURE A-10 Average transition rate (and standard error) vs. lane number for I-71 SB. (a) All stations onset; (b) All stations clearance

Statistical proof of such relationships between dependent variables and explainable variables requires more sample-day data to achieve more queues at the four sites studied in the body of this report.

\section{SITE: I-71 NB, OH, U.S.A.}

\section{Site description:}

Figure A-11 shows the schematic of northbound Interstate 71 near Columbus, Ohio. A major non-recurrent bottleneck (as labeled in the figure) activates during afternoon rush periods, and 
the resulting queues propagate backward for about half an hour. There are seven loop detector stations throughout the stretch, as shown in the figure. The distances between loop detector stations are different, from 500 meters to two kilemeters, and there are many on- and off-ramps during this long distance. Data from each detector consist of event-based speed. Speed contours and time-speed plots are drawn from raw speed data, and these data were aggregated at 30second sampling intervals for more efficient handling. This site is suitable to study the effect of merges and diverges on transition durations. Data from one sample day on July 20, 2007, were analyzed. More sample days would be introduced in the near future depends on the results of this sample day.

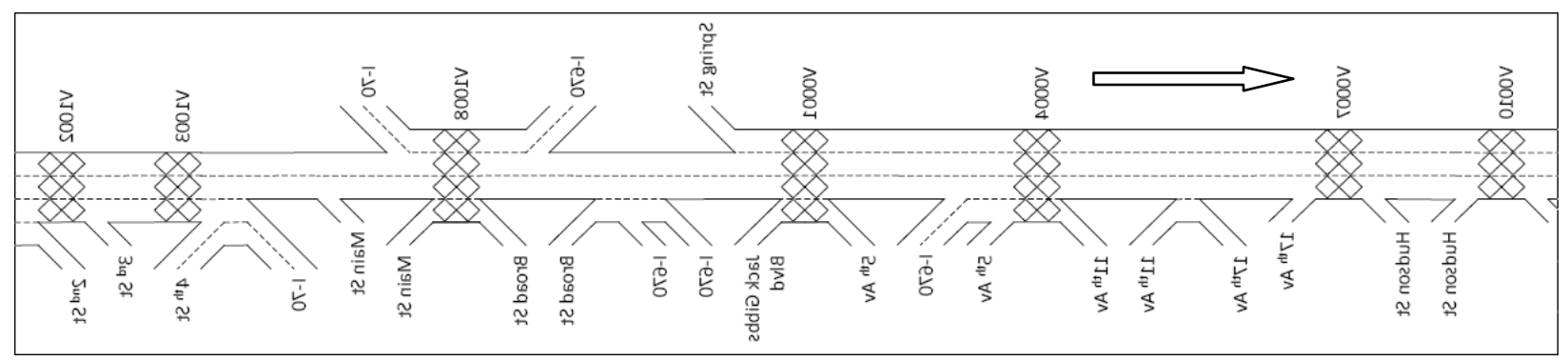

FIGURE A-11 Schematic of study site I-71 (NB), OH, U.S.A.

\section{Speed contours:}

Speed contours were constructed to assess general conditions on the study section. The all-lane speed contour of I-71 NB from 12-8 p.m.is presented in Figure A-12 as an example. As noted in the figure's legend, speed lower than $20 \mathrm{mph}$ is shaded in brown scale generated from the raw event-based speed. It shows a slight accident that propagated from station 7 to station 22 from 56 p.m. The figure illustrates a shock wave (brown band in the figure) that propagated backward. 


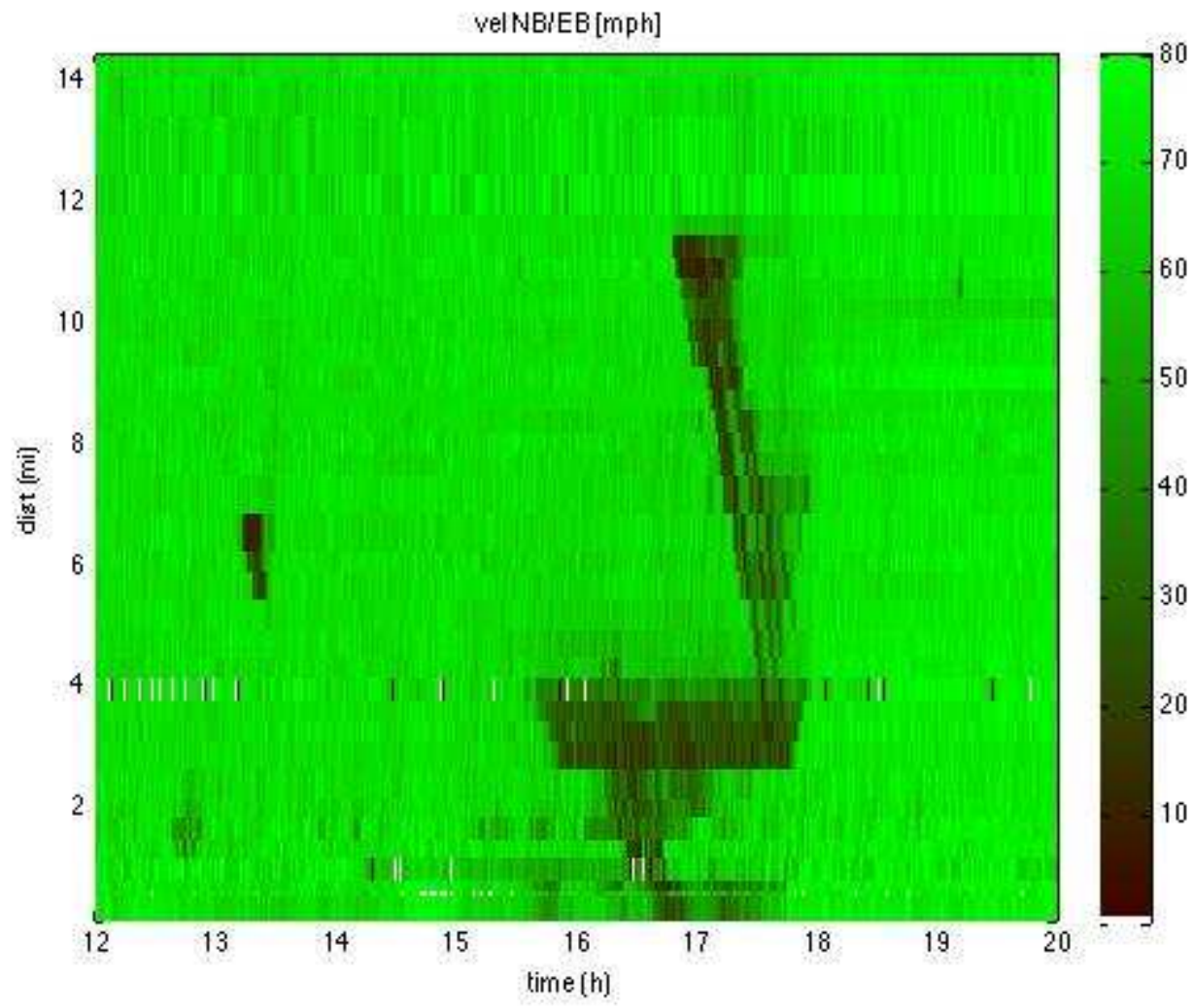

FIGURE A-12 Speed contour (all lanes) of I-71 NB from 12-8 p.m., July 20, 2007 


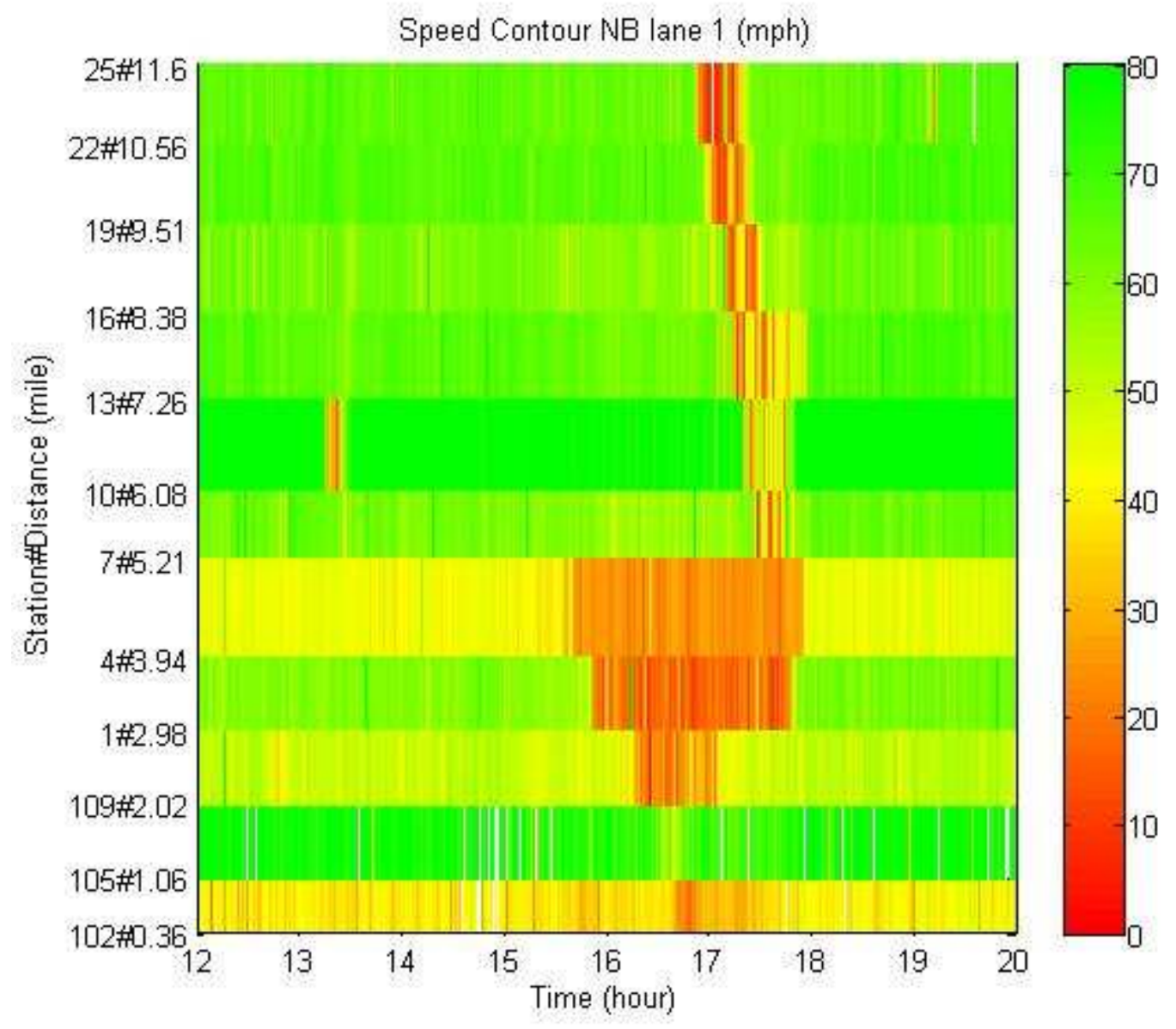

FIGURE A-13 Speed contour (lane 1) of I-71 NB from 12-8 p.m., July 20, 2007 


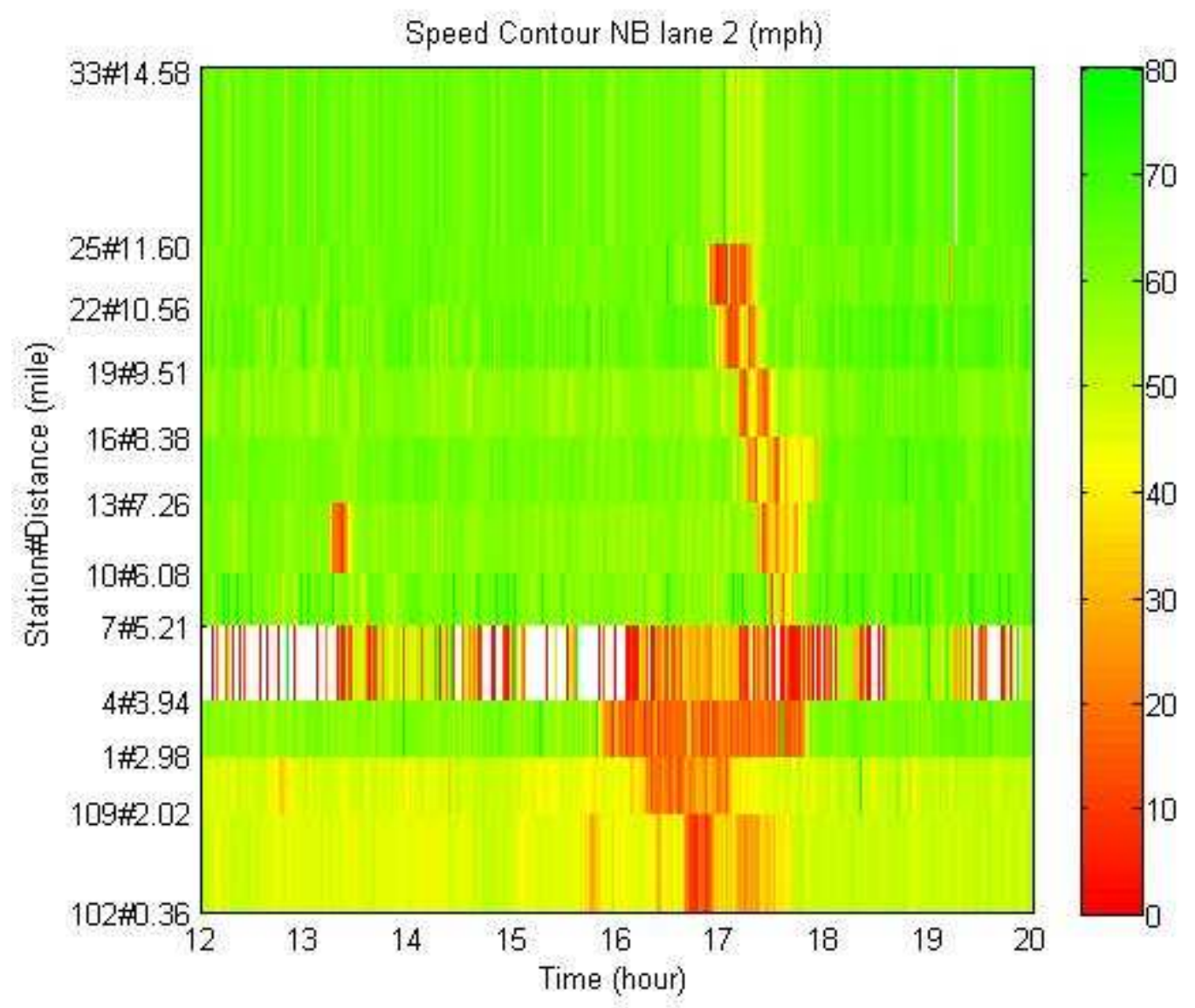

FIGURE A-14 Speed contour (lane 2) of I-71 NB from 12-8 p.m., July 20, 2007 


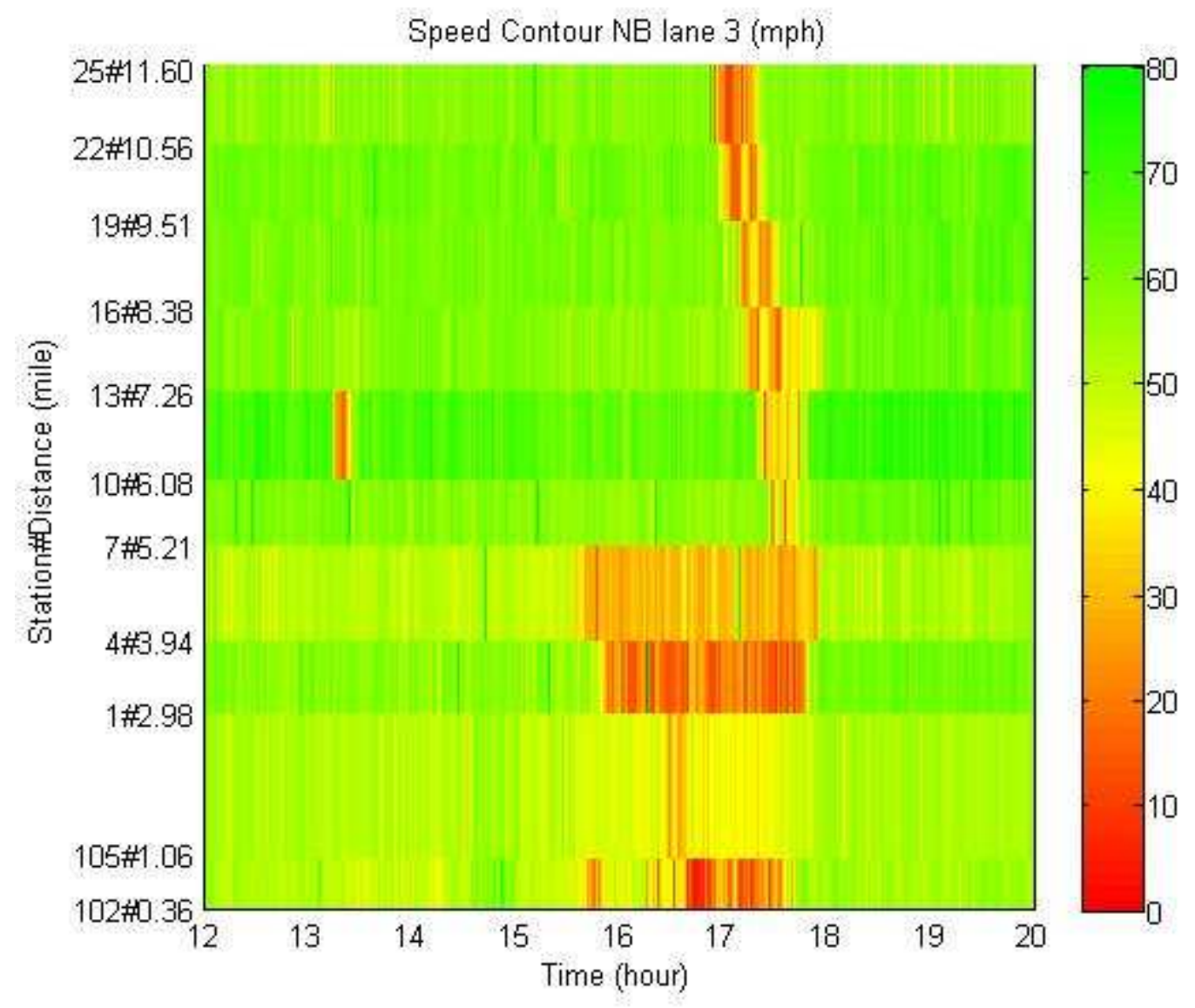

FIGURE A-15 Speed contour (lane 3) of I-71 NB from 12-8 p.m., July 20, 2007 


\section{Time series plots at each station at each lane:}
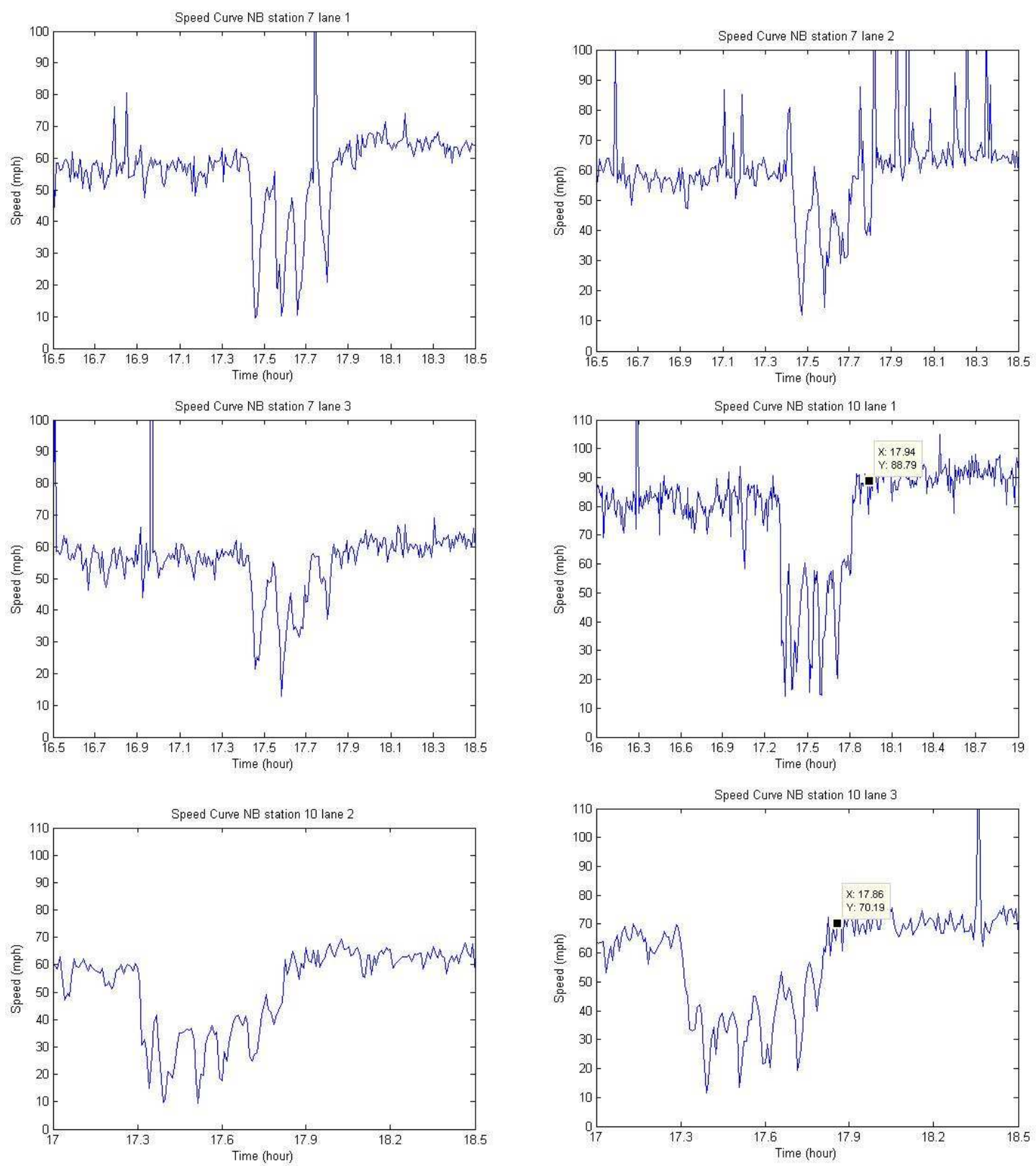

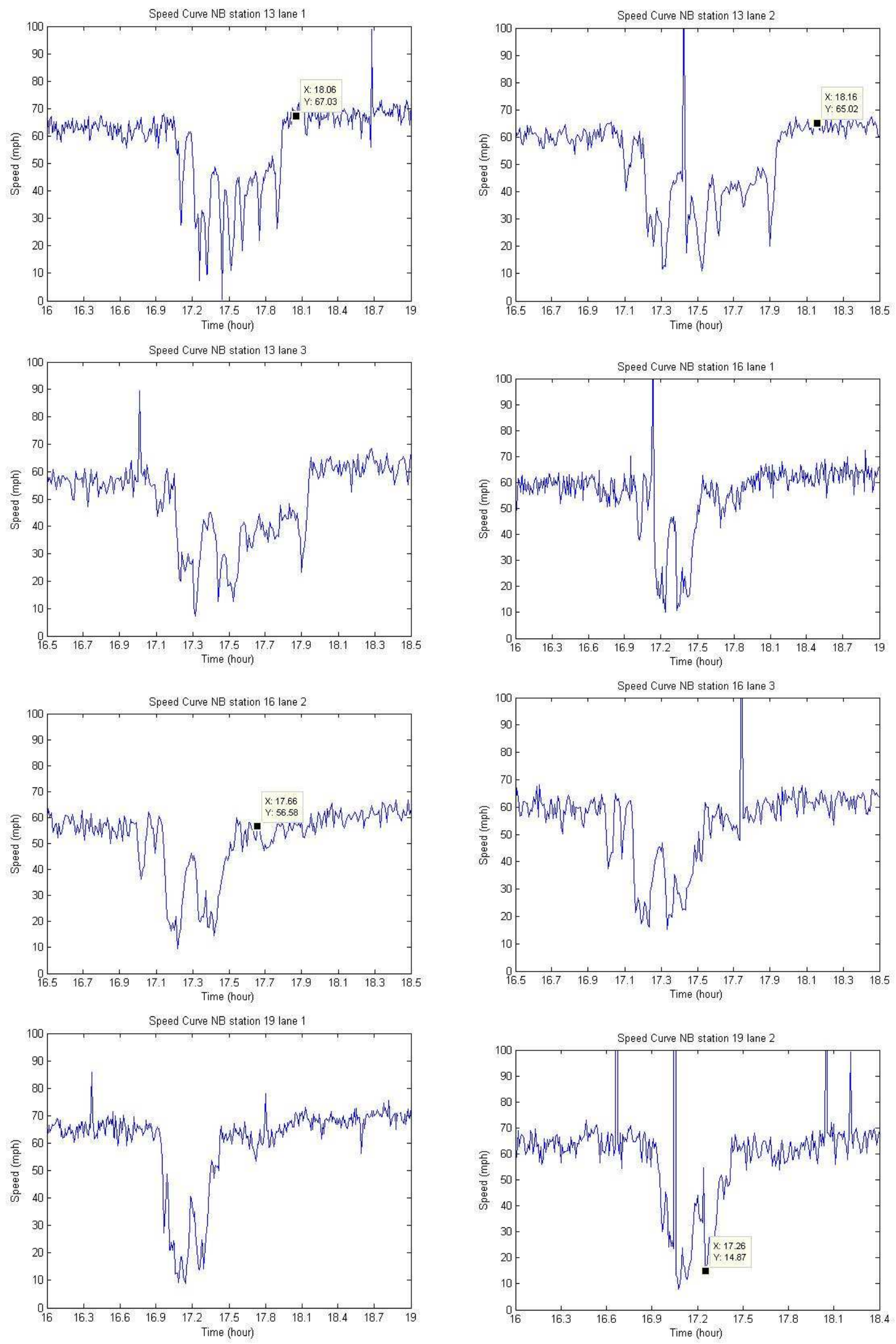

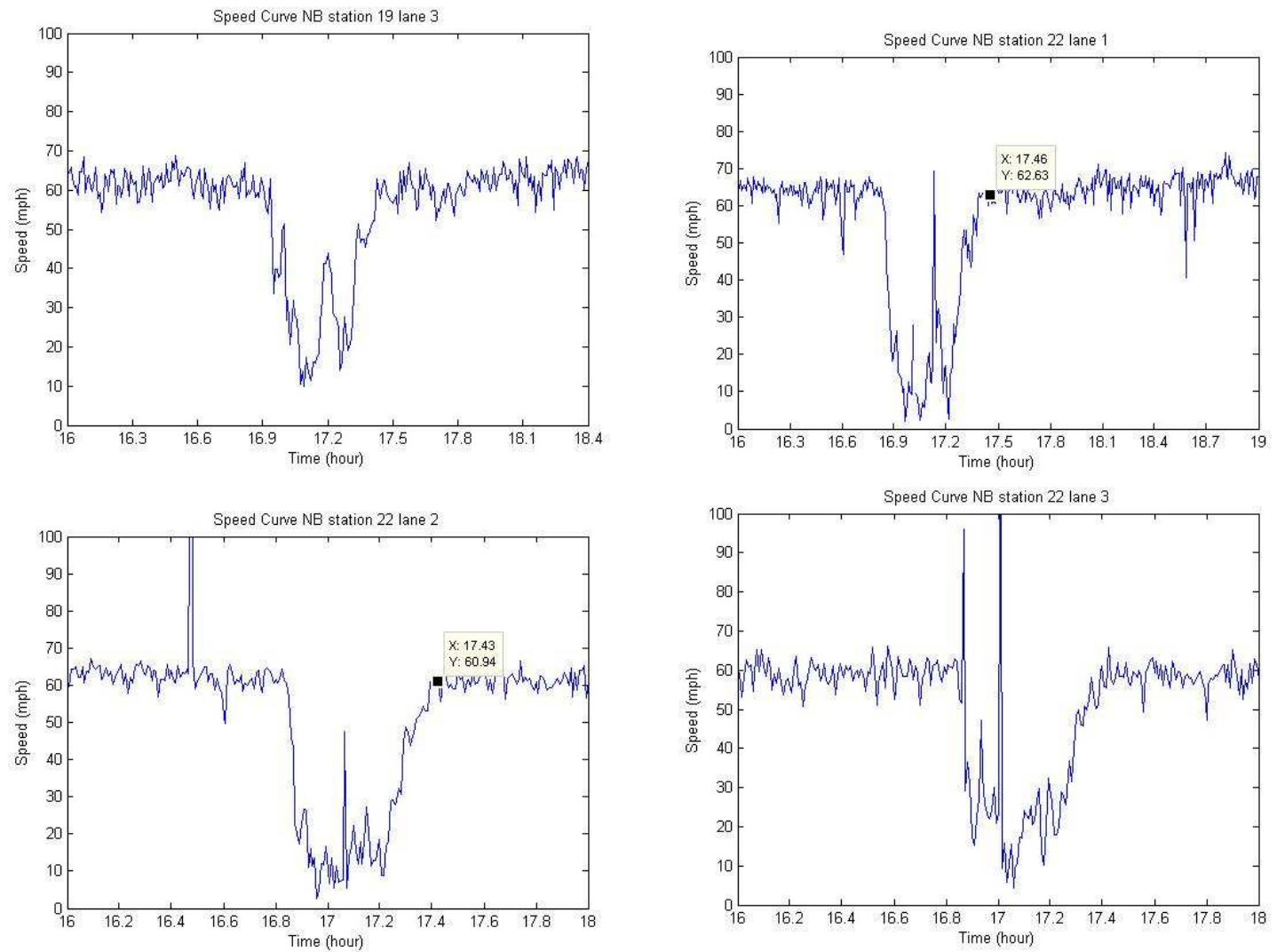

FIGURE A-16 Speed vs. Time (for each lane at each station) of I-71 NB from 5-6 p.m., July 20, 2007 


\section{Results:}

In order to analyze the relationships between dependant variables (transition duration and rates) and explanatory variables (initial speed, end speed, speed reduction and wave speed, etc.), data are calculated according to the time-speed plots for each lane at each station. The results are shown below:

Table A-3 dependant and explanatory variable data results of I-71 NB (Onset)

\begin{tabular}{|c|c|c|c|c|c|c|c|c|c|c|c|}
\hline $\begin{array}{c}\text { Lan } \\
\mathrm{e}\end{array}$ & $\begin{array}{c}\text { Statio } \\
\mathrm{n}\end{array}$ & $\begin{array}{l}\text { Milepo } \\
\text { st (km) }\end{array}$ & $\begin{array}{c}\text { Start } \\
\text { time( } \\
\text { h) }\end{array}$ & $\begin{array}{c}\text { End } \\
\text { time( } \\
\text { h) }\end{array}$ & $\begin{array}{l}\text { Duration( } \\
\mathrm{h})\end{array}$ & $\begin{array}{l}\text { Duration( } \\
\text { min.) }\end{array}$ & $\begin{array}{c}\text { Wave } \\
\text { speed } \\
(\mathrm{km} / \mathrm{h})\end{array}$ & $\begin{array}{c}\text { Start } \\
\text { speed }(\mathrm{km} / \\
\mathrm{h})\end{array}$ & $\begin{array}{c}\text { End } \\
\text { speed(k } \\
\mathrm{m} / \mathrm{h})\end{array}$ & $\begin{array}{c}\Delta \text { Speed } \\
(\mathrm{km} / \mathrm{h})\end{array}$ & $\begin{array}{c}\text { Transitio } \\
\text { n rate } \\
(\mathrm{km} / \mathrm{h} / \mathrm{mi} \\
\mathrm{n} .)\end{array}$ \\
\hline 1 & 7 & 8.38 & 17.42 & 17.46 & 0.04 & 2.4 & \multirow{6}{*}{-14.35} & 96.68 & 15.32 & -81.36 & -33.90 \\
\hline 1 & 10 & 9.79 & 17.29 & 17.34 & 0.05 & 3 & & 135.03 & 22.67 & -112.36 & -37.45 \\
\hline 1 & 13 & 11.69 & 17.18 & 17.26 & 0.08 & 4.8 & & 99.10 & 11.72 & -87.37 & -18.20 \\
\hline 1 & 16 & 13.49 & 17.13 & 17.17 & 0.04 & 2.4 & & 102.44 & 26.79 & -75.65 & -31.52 \\
\hline 1 & 19 & 15.32 & 16.93 & 17.01 & 0.08 & 4.8 & & 105.29 & 34.08 & -71.21 & -14.84 \\
\hline 1 & 22 & 16.99 & 16.82 & 16.97 & 0.15 & 9 & & 104.65 & 3.28 & -101.37 & -11.26 \\
\hline 2 & 7 & 8.38 & 17.43 & 17.48 & 0.05 & 3 & \multirow{6}{*}{-14.35} & 97.71 & 19.32 & -78.39 & -26.13 \\
\hline 2 & 10 & 9.79 & 17.29 & 17.34 & 0.05 & 3 & & 97.13 & 23.86 & -73.27 & -24.42 \\
\hline 2 & 13 & 11.69 & 17.2 & 17.23 & 0.03 & 1.8 & & 96.08 & 37.74 & -58.35 & -32.41 \\
\hline 2 & 16 & 13.49 & 17.12 & 17.18 & 0.06 & 3.6 & & 97.73 & 31.57 & -66.15 & -18.38 \\
\hline 2 & 19 & 15.32 & 16.93 & 17.01 & 0.08 & 4.8 & & 103.76 & 38.78 & -64.98 & -13.54 \\
\hline 2 & 22 & 16.99 & 16.83 & 16.93 & 0.1 & 6 & & 98.52 & 17.82 & -80.69 & -13.45 \\
\hline 3 & 7 & 8.38 & 17.43 & 17.46 & 0.03 & 1.8 & \multirow{6}{*}{-14.60} & 91.95 & 34.42 & -57.53 & -31.96 \\
\hline 3 & 10 & 9.79 & 17.29 & 17.39 & 0.1 & 6 & & 110.67 & 18.77 & -91.90 & -15.32 \\
\hline 3 & 13 & 11.69 & 17.2 & 17.23 & 0.03 & 1.8 & & 95.63 & 32.33 & -63.31 & -35.17 \\
\hline 3 & 16 & 13.49 & 17.13 & 17.16 & 0.03 & 1.8 & & 99.90 & 34.76 & -65.14 & -36.19 \\
\hline 3 & 19 & 15.32 & 16.93 & 17.07 & 0.14 & 8.4 & & 101.19 & 16.78 & -84.41 & -10.05 \\
\hline 3 & 22 & 16.99 & 16.84 & 16.91 & 0.07 & 4.2 & & 100.43 & 24.60 & -75.83 & -18.05 \\
\hline
\end{tabular}


Table A-4 dependant and explanatory variable data results of I-71 SB (Clearance)

\begin{tabular}{|c|c|c|c|c|c|c|c|c|c|c|c|}
\hline $\begin{array}{c}\text { Lan } \\
\mathrm{e}\end{array}$ & $\begin{array}{c}\text { Statio } \\
\mathrm{n}\end{array}$ & $\begin{array}{l}\text { Millia } \\
\text { ge } \\
(\mathrm{km})\end{array}$ & $\begin{array}{l}\text { Start } \\
\text { time }( \\
\text { h) }\end{array}$ & $\begin{array}{c}\text { End } \\
\text { time( } \\
\text { h) }\end{array}$ & $\begin{array}{c}\text { Duration( } \\
\text { h) }\end{array}$ & $\begin{array}{l}\text { Duration } \\
\text { (min.) }\end{array}$ & $\begin{array}{c}\text { Wave } \\
\text { speed } \\
(\mathrm{km} / \mathrm{h})\end{array}$ & $\begin{array}{c}\text { Start } \\
\text { speed }(\mathrm{k} \\
\mathrm{m} / \mathrm{h})\end{array}$ & $\begin{array}{c}\text { End } \\
\text { speed(km/ } \\
\text { h) }\end{array}$ & $\begin{array}{l}\Delta \text { Speed } \\
(\mathrm{km} / \mathrm{h})\end{array}$ & $\begin{array}{c}\text { Transitio } \\
\text { n rate } \\
(\mathrm{km} / \mathrm{h} / \mathrm{mi} \\
\mathrm{n} .)\end{array}$ \\
\hline 1 & 7 & 8.38 & 17.8 & 17.83 & 0.03 & 1.8 & \multirow{6}{*}{-14.85} & 33.81 & 94.33 & 60.52 & 33.62 \\
\hline 1 & 10 & 9.79 & 17.72 & 17.82 & 0.1 & 6 & & 32.75 & 134.76 & 102.01 & 17.00 \\
\hline 1 & 13 & 11.69 & 17.9 & 17.94 & 0.04 & 2.4 & & 42.50 & 106.32 & 63.82 & 26.59 \\
\hline 1 & 16 & 13.49 & 17.43 & 17.53 & 0.1 & 6 & & 25.62 & 90.82 & 65.21 & 10.87 \\
\hline 1 & 19 & 15.32 & 17.29 & 17.43 & 0.14 & 8.4 & & 22.88 & 99.56 & 76.68 & 9.13 \\
\hline 1 & 22 & 16.99 & 17.22 & 17.39 & 0.17 & 10.2 & & 4.05 & 102.14 & 98.09 & 9.62 \\
\hline 2 & 7 & 8.38 & 17.58 & 17.73 & 0.15 & 9 & \multirow{6}{*}{-23.92} & 23.41 & 93.70 & 70.29 & 7.81 \\
\hline 2 & 10 & 9.79 & 17.71 & 17.82 & 0.11 & 6.6 & & 40.04 & 99.35 & 59.31 & 8.99 \\
\hline 2 & 13 & 11.69 & 17.9 & 17.94 & 0.04 & 2.4 & & 32.33 & 95.25 & 62.92 & 26.22 \\
\hline 2 & 16 & 13.49 & 17.42 & 17.51 & 0.09 & 5.4 & & 23.20 & 81.61 & 58.41 & 10.82 \\
\hline 2 & 19 & 15.32 & 17.29 & 17.43 & 0.14 & 8.4 & & 26.53 & 106.58 & 80.05 & 9.53 \\
\hline 2 & 22 & 16.99 & 17.22 & 17.39 & 0.17 & 10.2 & & 14.15 & 98.23 & 84.08 & 8.24 \\
\hline 3 & 7 & 8.38 & 17.8 & 17.82 & 0.02 & 1.2 & \multirow{6}{*}{-14.85} & 59.92 & 95.63 & 35.71 & 29.76 \\
\hline 3 & 10 & 9.79 & 17.72 & 17.82 & 0.1 & 6 & & 31.28 & 116.35 & 85.07 & 14.18 \\
\hline 3 & 13 & 11.69 & 17.9 & 17.94 & 0.04 & 2.4 & & 37.38 & 96.31 & 58.93 & 24.55 \\
\hline 3 & 16 & 13.49 & 17.43 & 17.54 & 0.11 & 6.6 & & 35.77 & 99.53 & 63.76 & 9.66 \\
\hline 3 & 19 & 15.32 & 17.3 & 17.43 & 0.13 & 7.8 & & 31.99 & 101.33 & 69.34 & 8.89 \\
\hline 3 & 22 & 16.99 & 17.22 & 17.39 & 0.17 & 10.2 & & 28.92 & 96.60 & 67.68 & 6.64 \\
\hline
\end{tabular}

Once transition start and end times were identified, various explanatory variables were measured to identify factors that influence transition durations along shock waves. Unlike the other four sites, there is only a one-day sample of data for this site. Thus, only the bivariate relations between transition duration and different lanes or stations are analyzed for this site. Statistical significance of influence of each explanatory variable will be studied in the future.

\section{Findings:}

The observed individual transition durations of NB I-71 on this sample day range from two to eight minutes for onset and one to 10 minutes for clearance. The observed individual transition rates range from -12.81 to $-30.66 \mathrm{~km} / \mathrm{h} / \mathrm{min}$ for onset, and from 8.16 to $25.79 \mathrm{~km} / \mathrm{h} / \mathrm{min}$ for clearance.

\section{Effect of distance to the bottleneck on transition duration}

Figures A-17 (a) and (b) show plots of average transition durations of all lanes (red circles) vs. stations located upstream of the bottleneck. The vertical lines crossing each circle represent the bounds of standard error. The average duration is 4.03 minutes for the onset with the standard error of 1.58 minutes, and the average duration is 6.17 minutes for the clearance with the standard error 0.94 minutes. It appears that the average durations do not change significantly longitudinally over space for the onset but significantly for the clearance. 


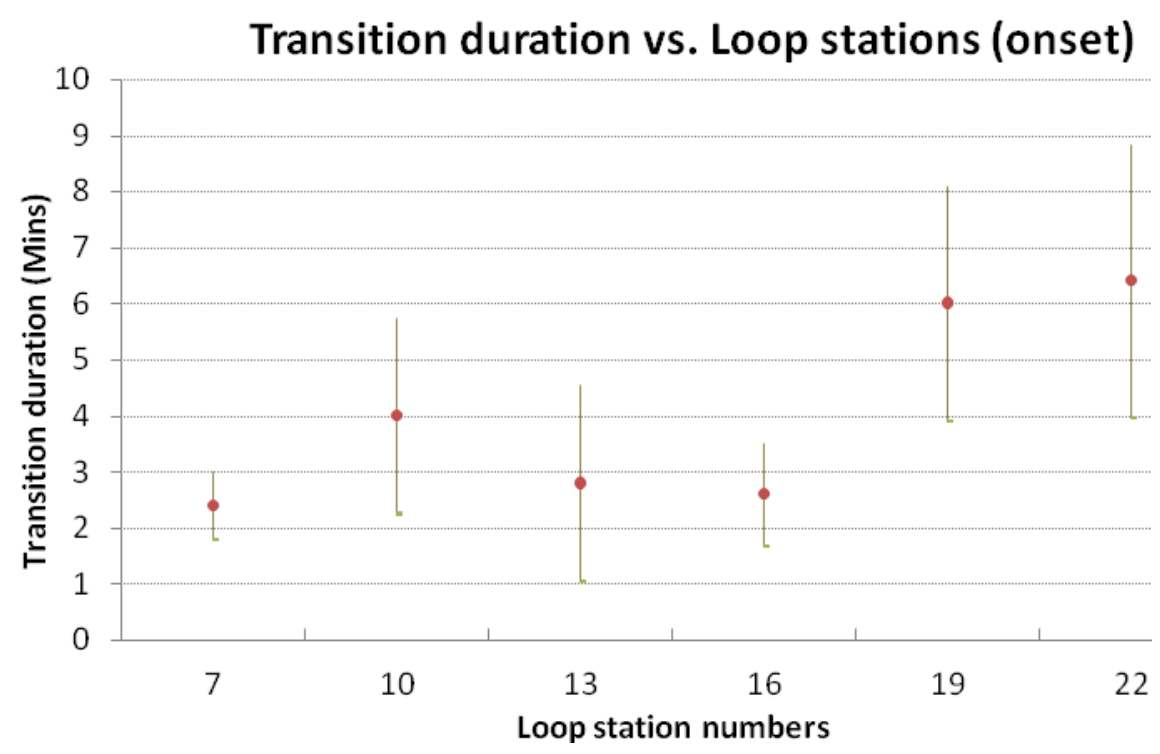

(a)

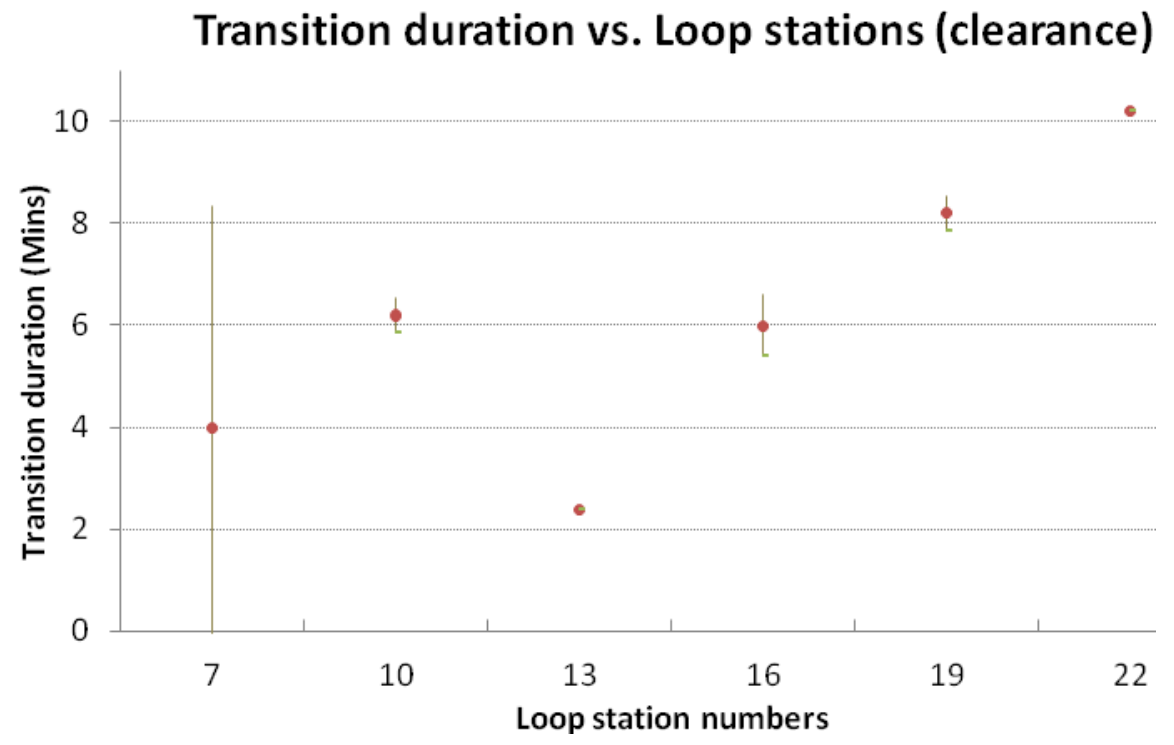

(b)

FIGURE A-17 Average transition duration (and standard error) vs. detector station for I71 NB (a) all lanes onset; (b) all lanes clearance.

\section{Effect of lanes on transition duration}

In Figure A-18 (a) and (b), the average durations and their standard error bounds are presented by lane (with data from the seven stations combined). Figure A-18 (a) and (b) show that the average durations of both onset and clearance change little across lanes; only the average duration of onset is a little lower (2.14 minutes) than that of clearance. 


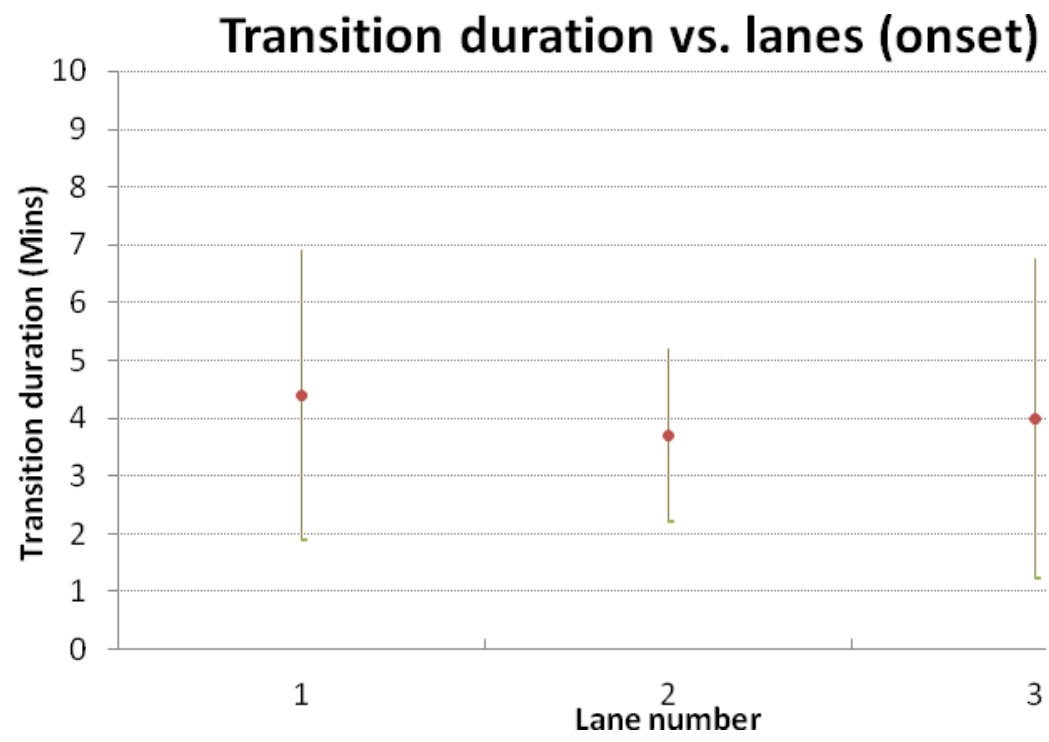

(a)

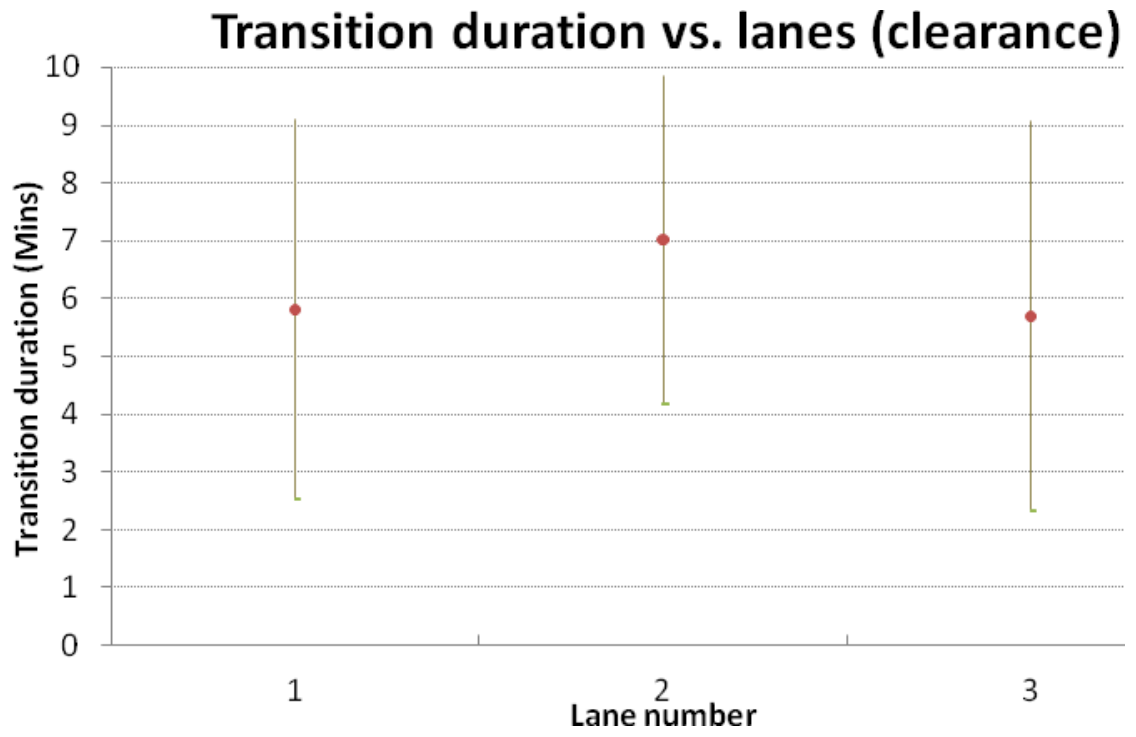

(b)

FIGURE A-18 Average transition duration (and standard error) vs. lane number for I-71 NB.

(a) All stations onset; (b) All stations clearance

\section{Effect of distance to the bottleneck on transition rate}

Figures A-19 (a) and (b) show plots of average transition rates of all lanes (red circles) vs. stations located upstream of the bottleneck. The vertical lines crossing each circle represent the bounds of standard error. The average rate is $-23.46 \mathrm{~km} / \mathrm{h} / \mathrm{min}$ for the onset with the standard error of $6.58 \mathrm{~km} / \mathrm{h} / \mathrm{min}$, and average rate is $15.12 \mathrm{~km} / \mathrm{h} / \mathrm{min}$ for the clearance with the standard error of $3.60 \mathrm{~km} / \mathrm{h} / \mathrm{min}$. It appears that the average rates do not change significantly longitudinally over space for the onset but seem significant for the clearance. 


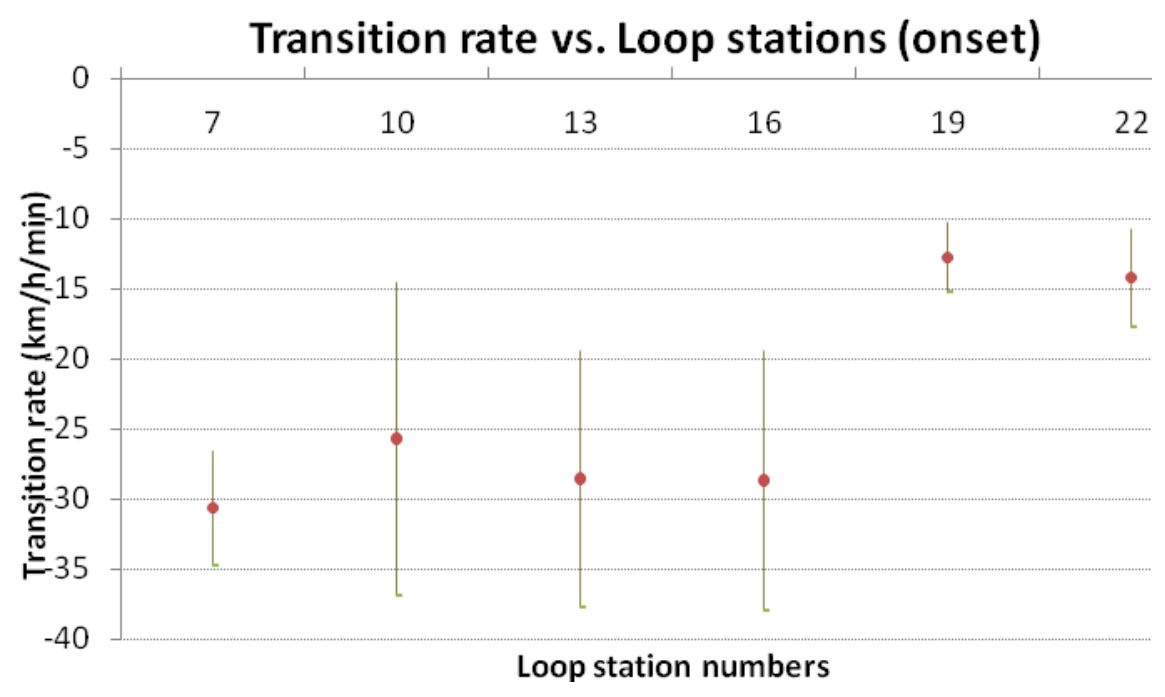

(a)

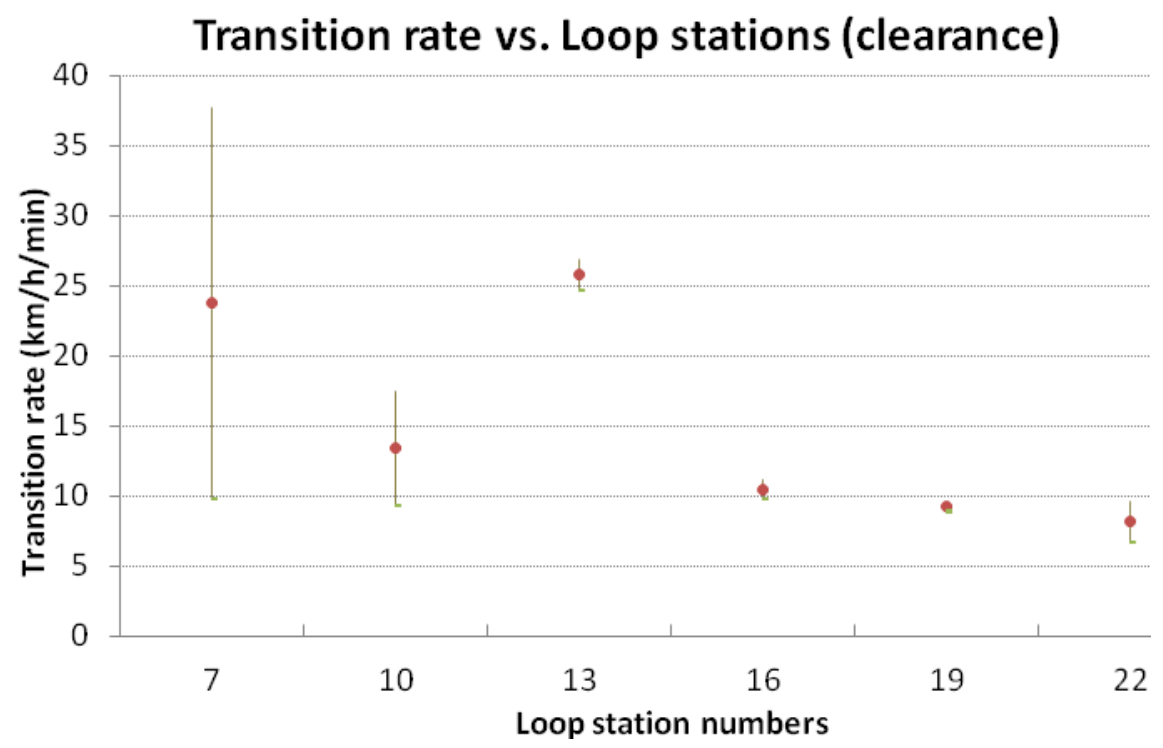

(b)

FIGURE A-19 Average transition rate (and standard error) vs. detector station for I-71 NB (a) all lanes onset; (b) all lanes clearance.

\section{Effect of lanes on transition rate}

In Figure A-20 (a) and (b), the average rates and their standard error bounds are presented by lane (with data from the seven stations combined). Figure A-20 (a) and (b) show that the average rates of both onset and clearance change little across lanes; only the average rate of onset is longer $(8.34 \mathrm{~km} / \mathrm{h} / \mathrm{min}$ in obsolete value $)$ than that of clearance. 


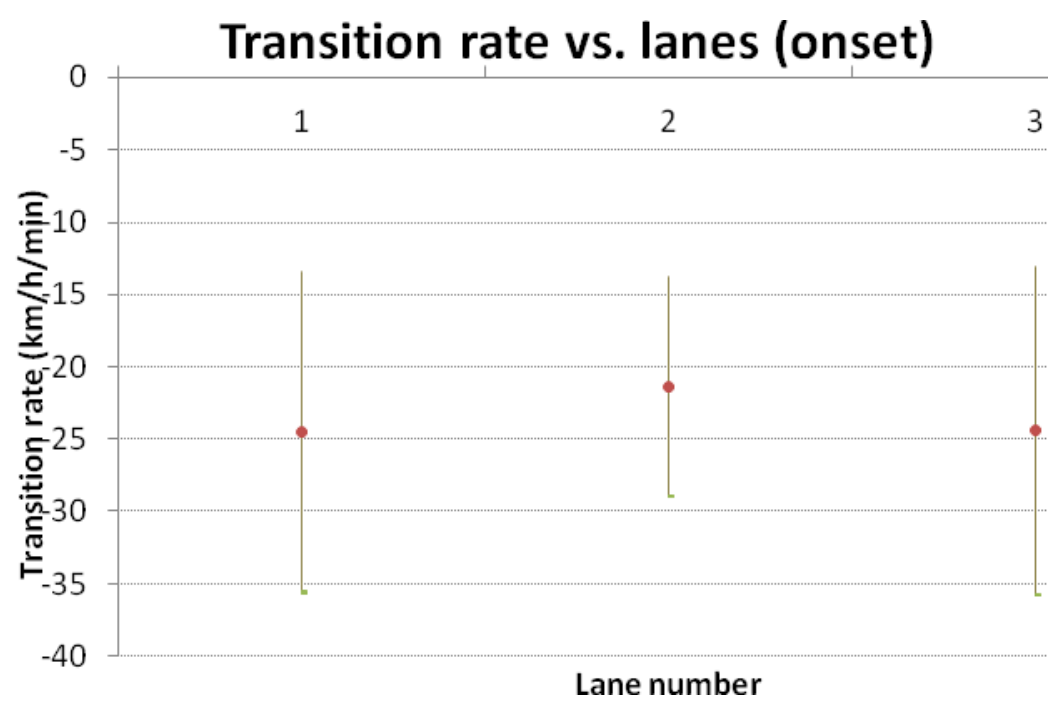

(a)

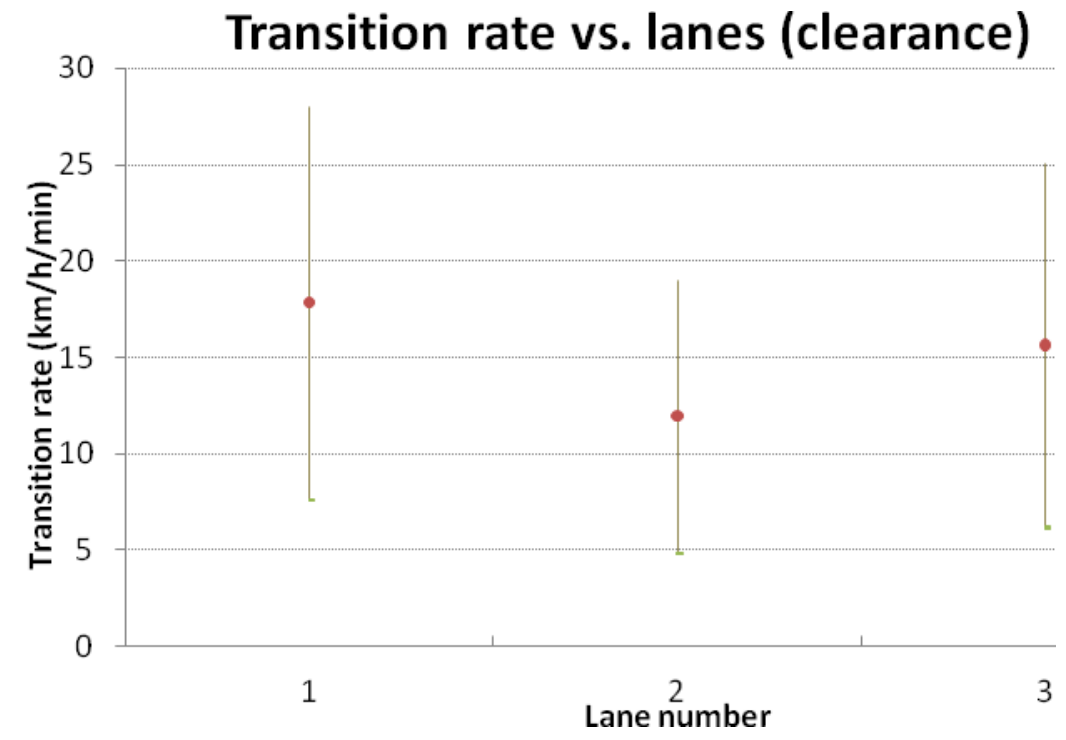

(b)

FIGURE A-20 Average transition rate (and standard error) vs. lane number for I-71 NB. (a) All stations onset; (b) All stations clearance

Statistical proof of such relationships between dependent variables and explainable variables requires more sample-day data to achieve more queues at the four sites studied in the body of this report. 


\section{SITE: A2-SB, EVERDINGEN, UTRECHT, HOLLAND.}

Data type: $\quad$ Individual trajectory data captured by helicopter

Data resolution: $\quad$ Digital black and white camera (1400 x 1392); Images are collected at $11.6 \mathrm{~Hz}$.

Data process: $\quad$ aggregate in forms of $50 \mathrm{~m}$ by 1 second to achieve spatial-mean speed and density.

\section{Site description:}

This site is located in A2 southbound, near Everdingen, Utrecht, in Holland. A nonrecurrent bottleneck near an off-ramp activates during afternoon rush hour periods, causing a traffic jam that propagates backwards. The study egment is divided into 18 sections of 50 meters each. The original data resolution is 0.1 second, and aggregation leads to $50 \mathrm{~m} * 0.1 \mathrm{sec}$ time-space speed plots, as shown as below.

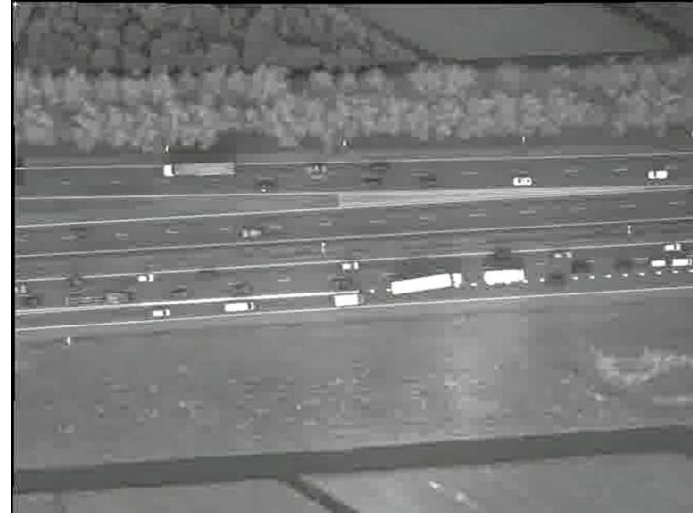

(a)

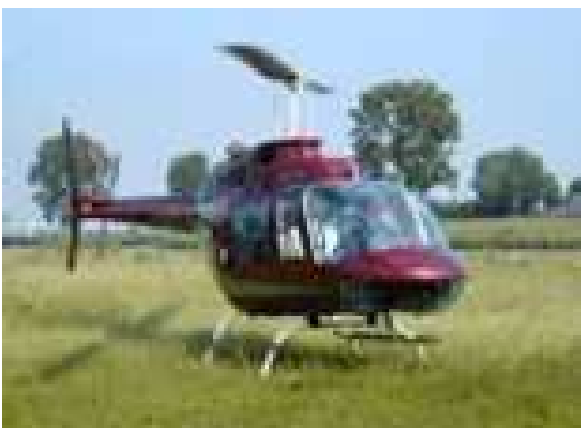

(b)

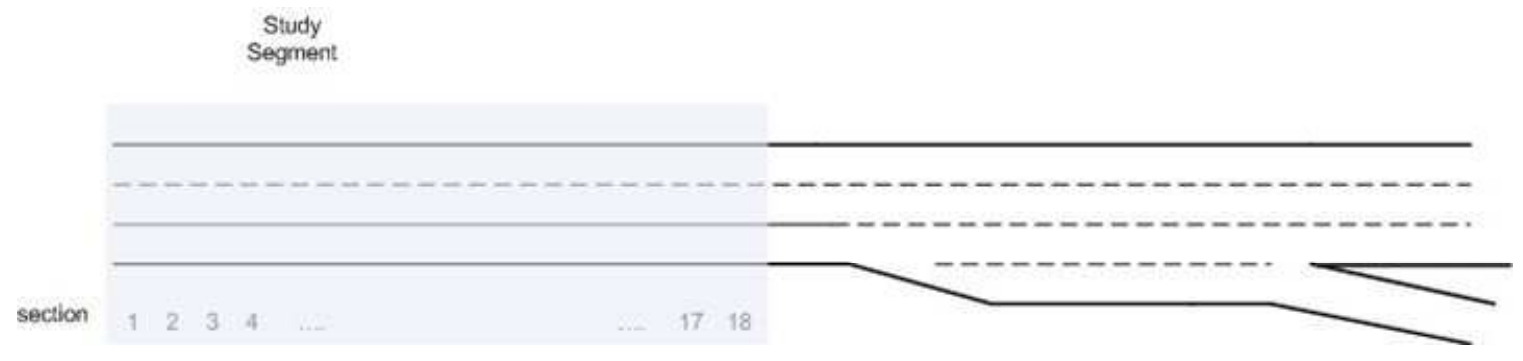

(c)

FIGURE A-21 (A) The layout of the study segment (B) Helicopter for data collection (C) Scheme of the segment. 


\section{Speed contours:}

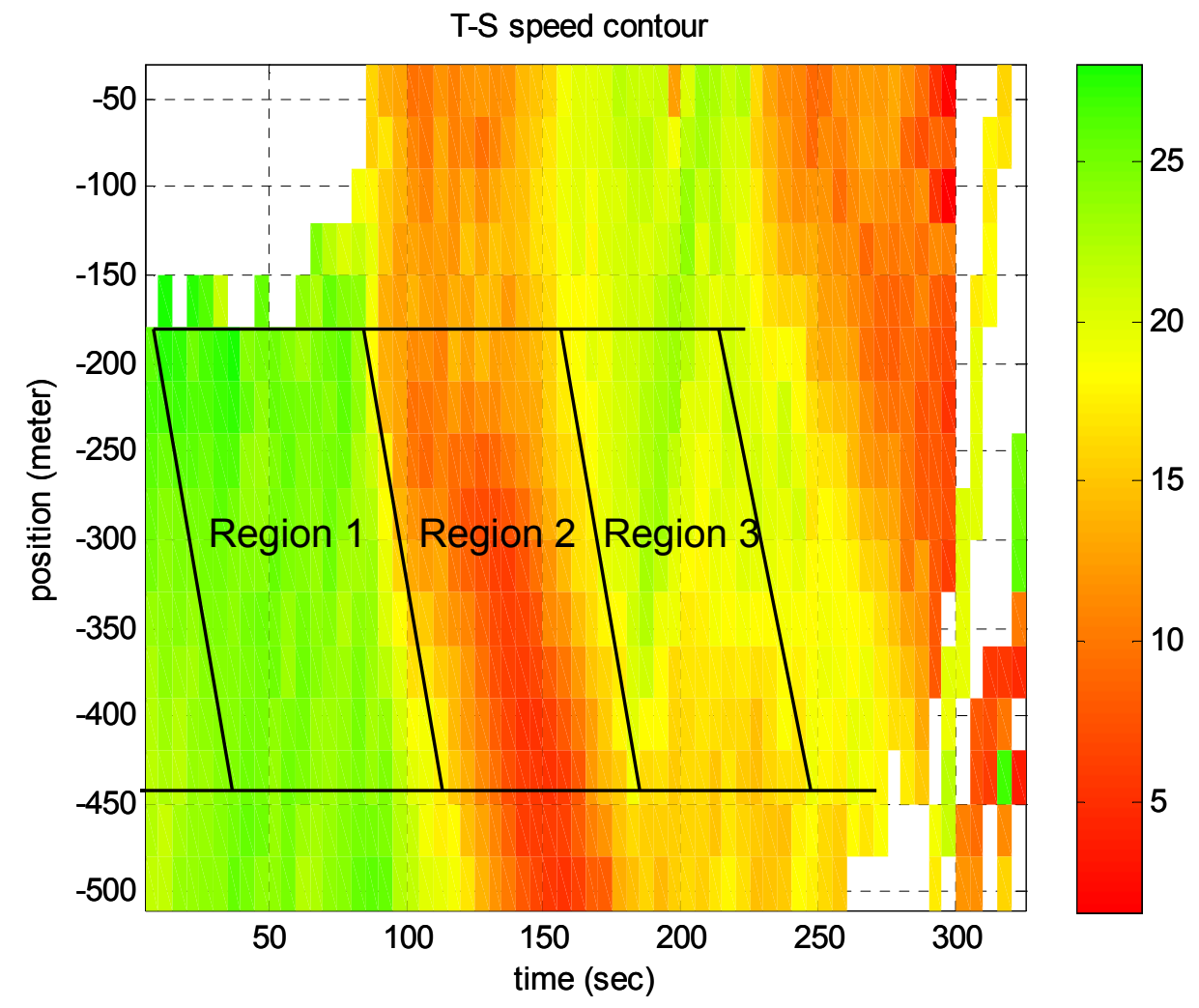

FIGURE A-22 Time-space speed (meter/sec) contour plots on Dutch site A2, collected and generated by helicopter. 


\section{Time series plots at each region:}
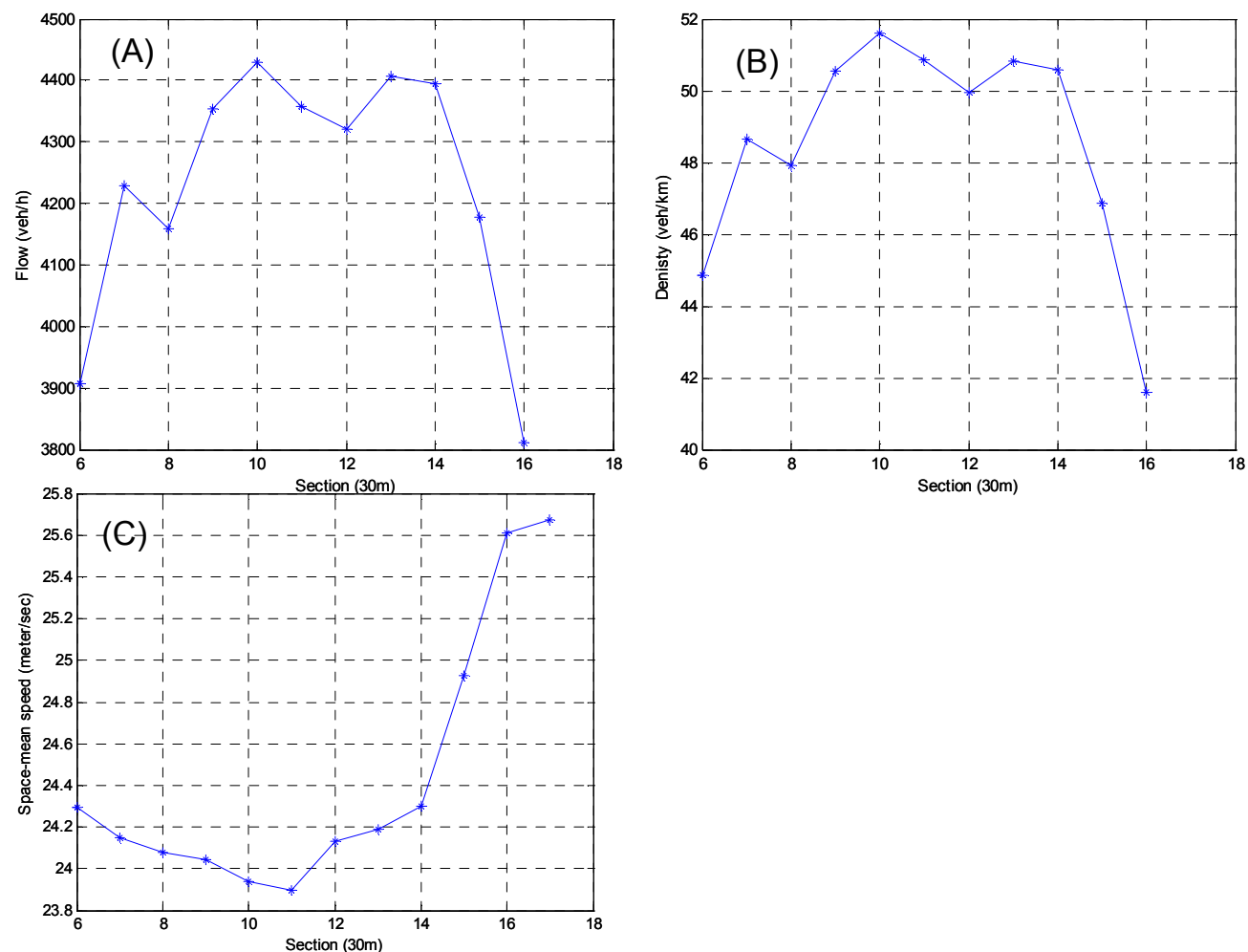

FIGURE A-23: Region 1--Flow, Density and Space-mean speed vs Freeway section on Dutch site 

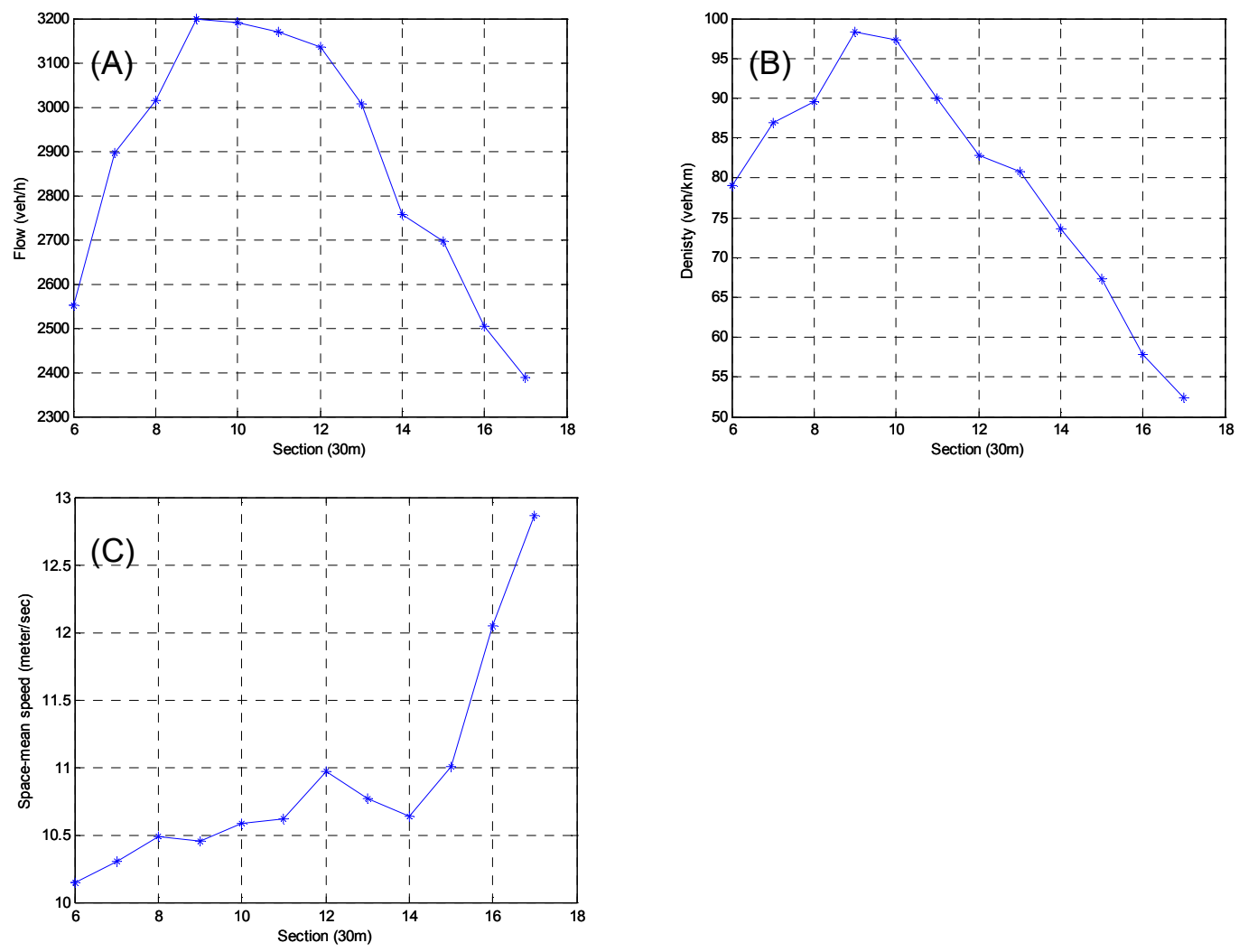

FIGURE A-24: Region 2--Flow, Density and Space-mean speed vs Freeway section on Dutch site 

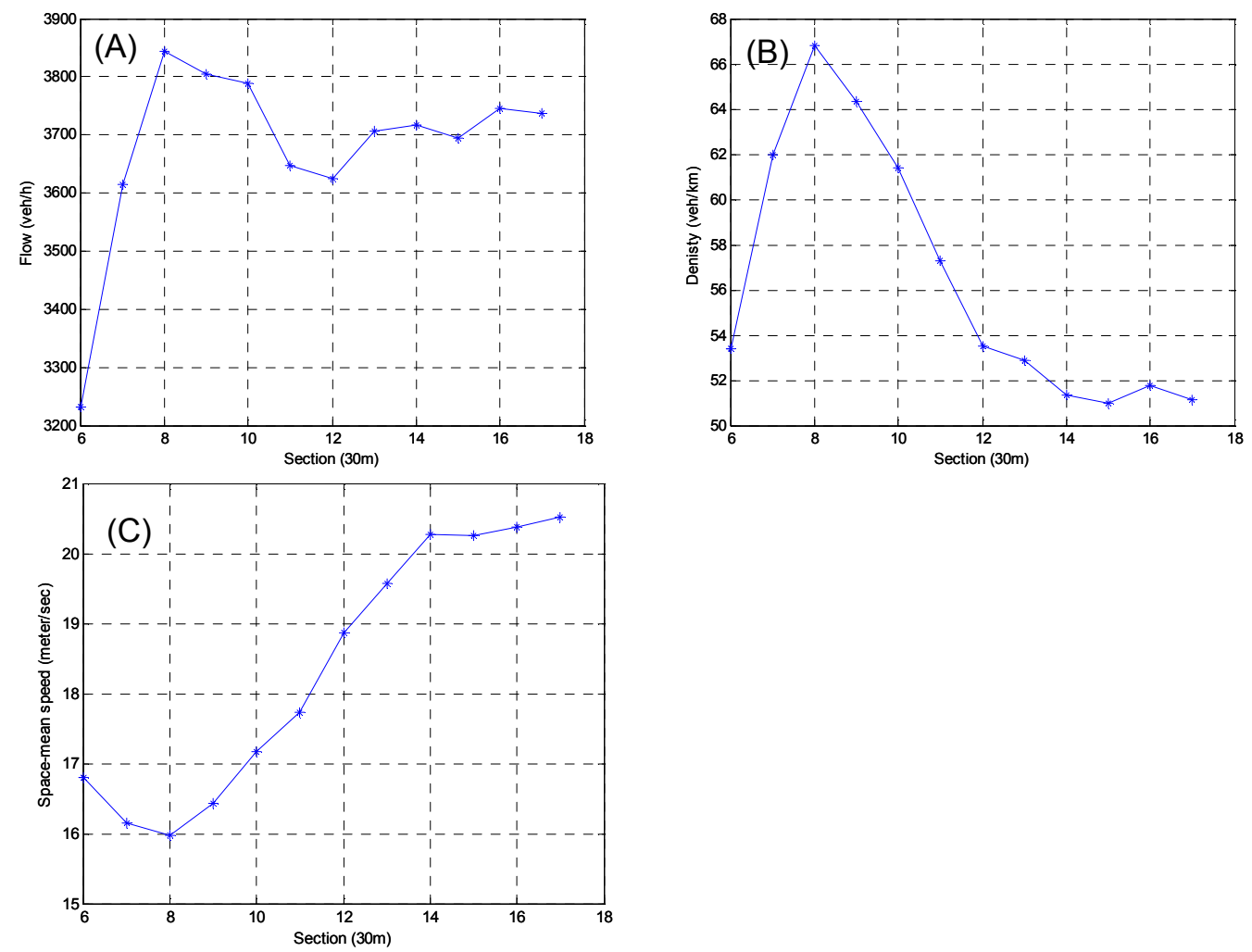

FIGURE A-25: Region 3--Flow, Density and Space-mean speed vs Freeway section on Dutch site
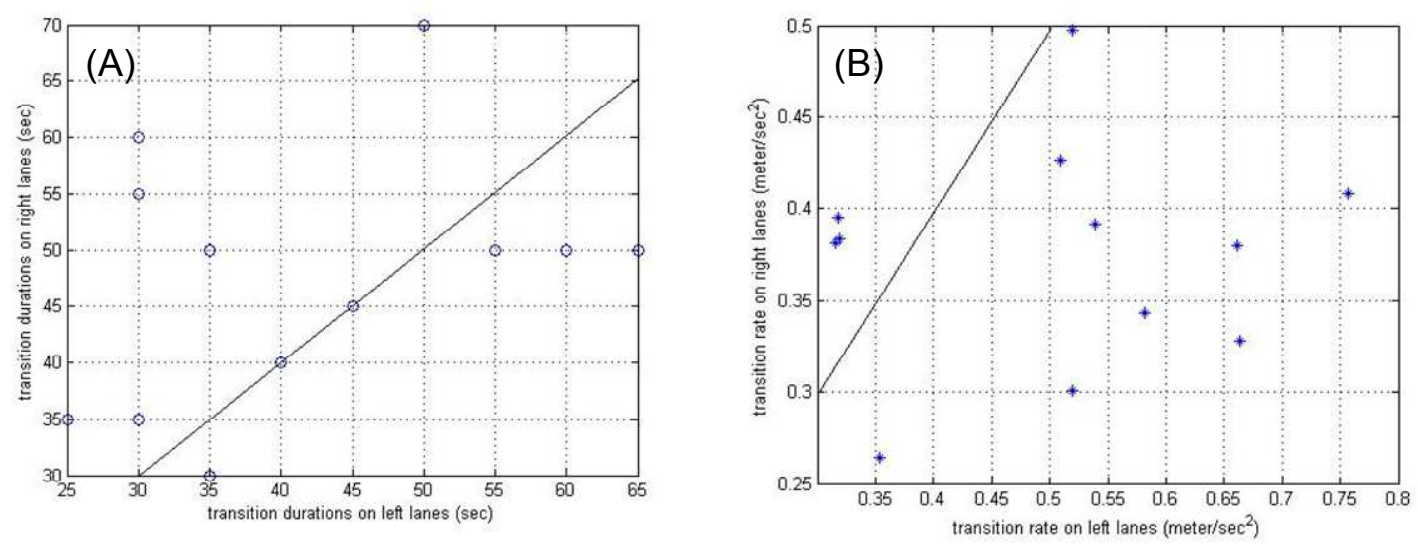

FIGURE A-26: Dutch Site Analysis. (A) transition durations on left lanes and right lanes; (B) transition rates on left lanes and right lanes

\section{Results:}

The figures from A-23 to A-25 show the changes of flow, density and speed over sections, but no appropriate explanation for the results can be given for now.

Figure B-26 attempts to find the transition difference in different lanes. For this purpose, a simple Chi-square hypothesis is given below: 


\begin{abstract}
(A) Hypothesis test on transition durations

H0: Transition duration on the left lane = transition duration on right lane

H1: There is significant difference between the transition durations on the left and right lanes.

Chi-Square test: 6 points fall into the left plane, and 5 points fall into the right plane. Chi Square $=0.091$ and $\mathrm{P}$-value $=0.95$.

Therefore, $\mathrm{H} 0$ is accepted.
\end{abstract}

\title{
(B)
}

\section{Hypothesis test on transition rate}

H0: Transition duration on the left lane = transition duration on the right lane

H1: There is significant difference between the transition durations on the left and right lanes

Chi-Square test: 3 points fall into the left plane, and 9 points fall into the right plane. Chi Square $=3$ and $\mathrm{P}$-value $=0.07$.

Therefore, $\mathrm{H} 1$ is accepted. (H1 is accepted under $90 \%$ confidence circumstances considering only very few data available)

[avergage transition from the left to the right]

$=\left[\begin{array}{ll}41.667 & 47.500\end{array}\right](\mathrm{sec})$

[Average transition rate from the left to the right]

$=[2.52421 .8743]\left(\right.$ meter $\left./ \mathrm{sec}^{\wedge} 2\right)$

\section{Findings:}

No substantial findings about traffic state changes over sections are given for now. More microscopic data may be required for further analysis. The most differences lie between microscopic data and loop detector data. Transition durations obtained from loop detectors range from four minutes up to 11 minutes, while transition durations obtained from microscopic trajectory data are only less than one minute.

$\begin{array}{lll} & \text { left-lanes }==\text { right-lanes } \\ \text { Transition durations } & \text { YES } & \mathrm{P}=0.95 \\ \text { Transition rates } & \text { NO } & \mathrm{P}=0.07\end{array}$

[avergage transition from the left to the right] $=\left[\begin{array}{ll}41.667 & 47.500\end{array}\right](\mathrm{sec})$

[Average transition rate from the left to the right] $=\left[\begin{array}{ll}2.5242 & 1.8743\end{array}\right]\left(\right.$ meter $\left./ \mathrm{sec}^{\wedge} 2\right)$

According to the above result, it is clearly shown that transition durations have no significant difference between lanes, but transition rates do under $90 \%$ confidence circumstances. The transition rates decrease from the left lane to the right lane. 


\section{SITE: I-405 NB AT MULHOLLAND DRIVE, LOS ANGELES, CA}

Data type: Individual trajectory data

Data resolution: $\quad 0.1 \mathrm{sec}$

Data process: $\quad$ aggregate in forms of 50 meters by 30 seconds to achieve spatial-mean speed and density.

\section{Site description:}

This site is an upgrade measuring 1,341 feet long. The section is approximately one-half mile from the top of a 2.5-mile long grade. The level of service was in the $\mathrm{C}$ to $\mathrm{E}$ range. The site consists of a tangent section with five 11-foot lanes and a narrow left shoulder.

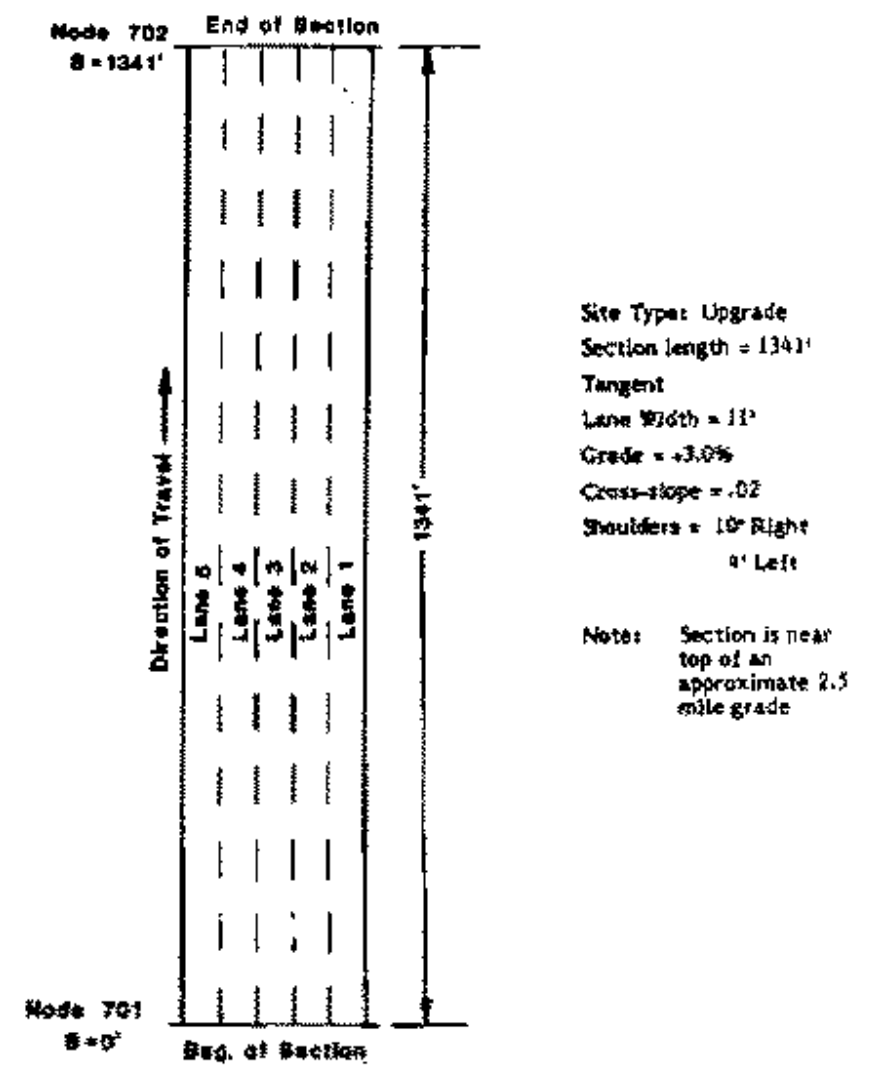

NOF TO SCALE

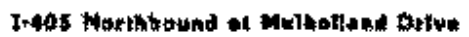

FIGURE A-27: The layout of study road segment on I-405 NB. 
Speed contours:

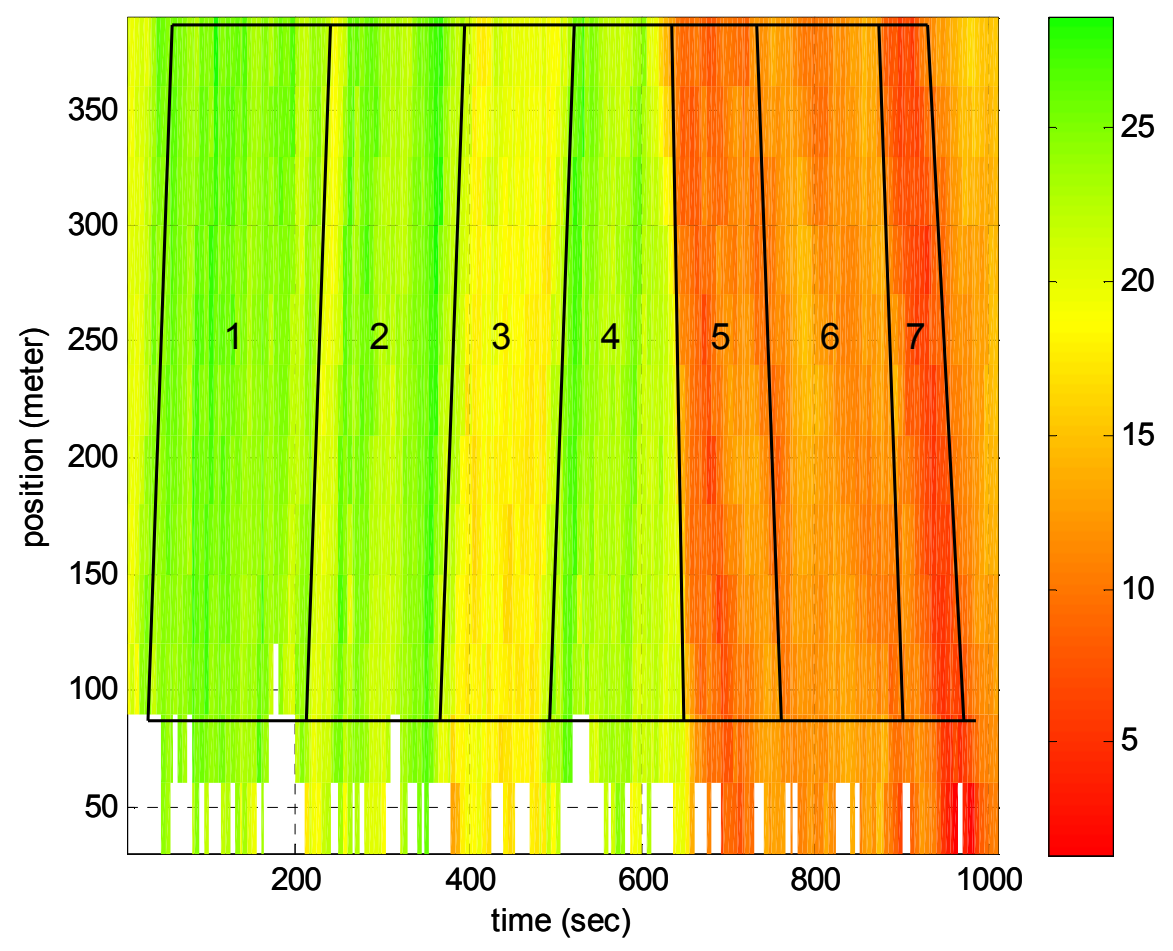

FIGURE A-28: Time-space speed contour plots on CA site I-405NB

Time series plots at each region: 

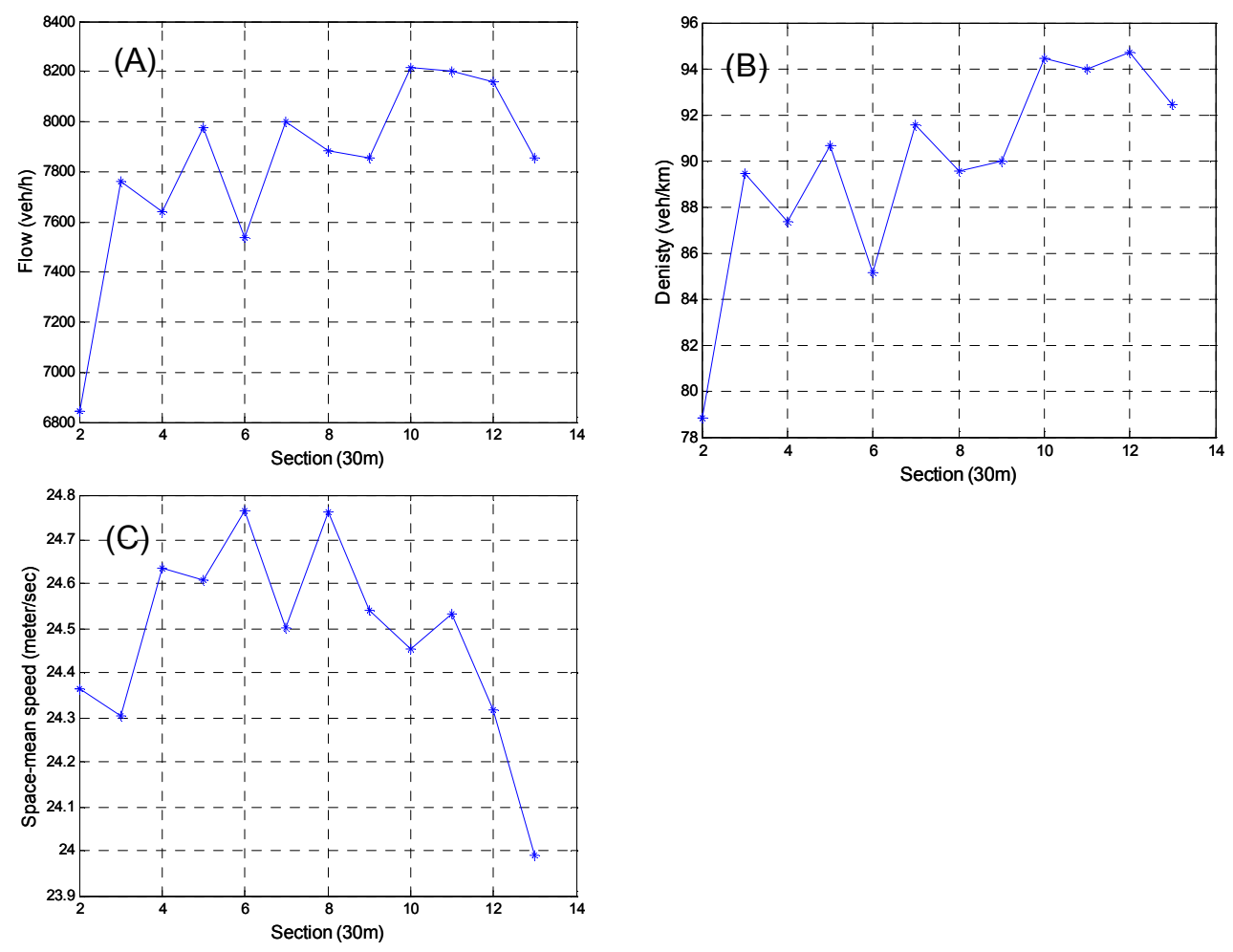

FIGURE A-29: Region 1--Flow, Density and Space-mean speed vs Freeway section on I405NB
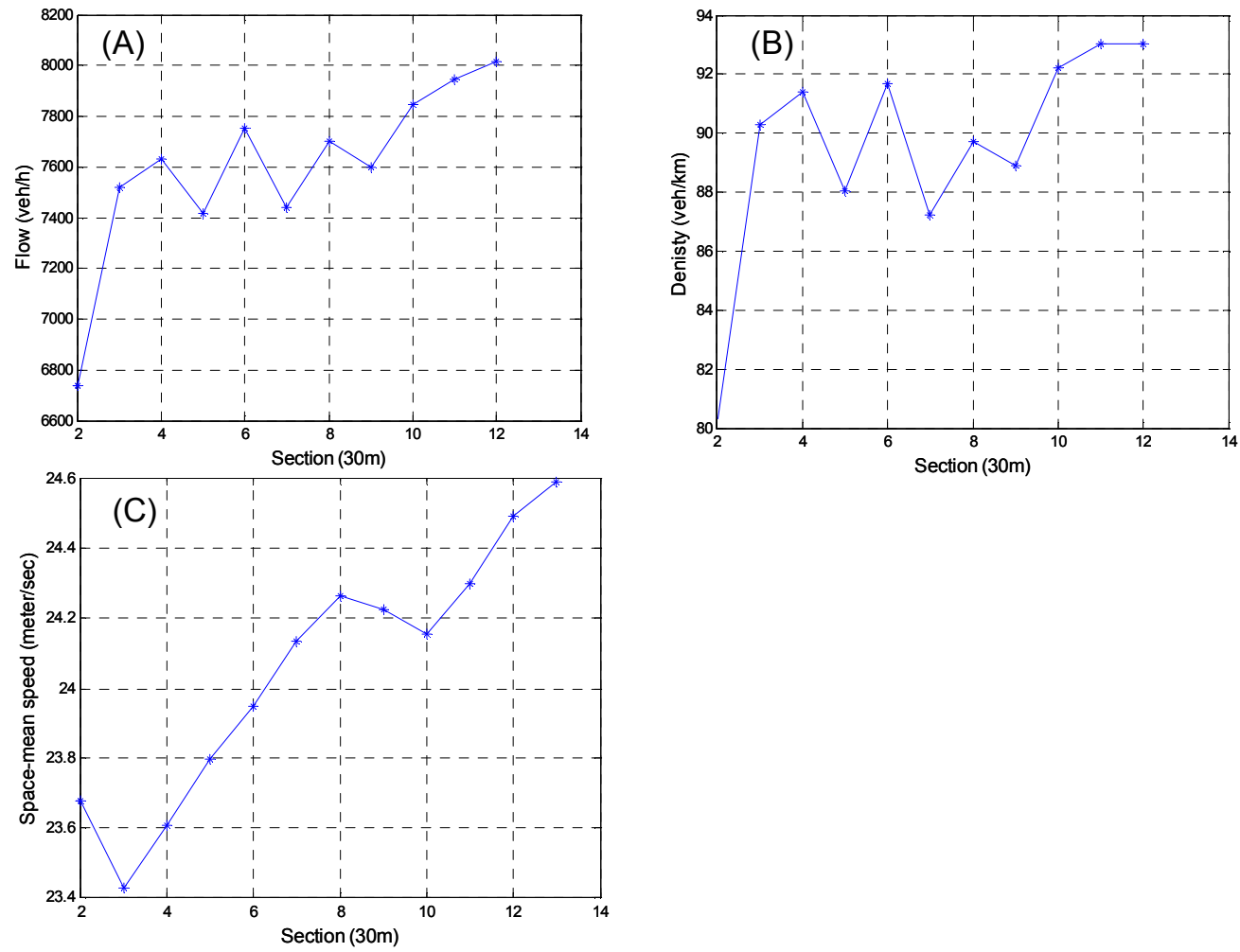

FIGURE A-30: Region 2--Flow, Density and Space-mean speed vs Freeway section on I405NB 

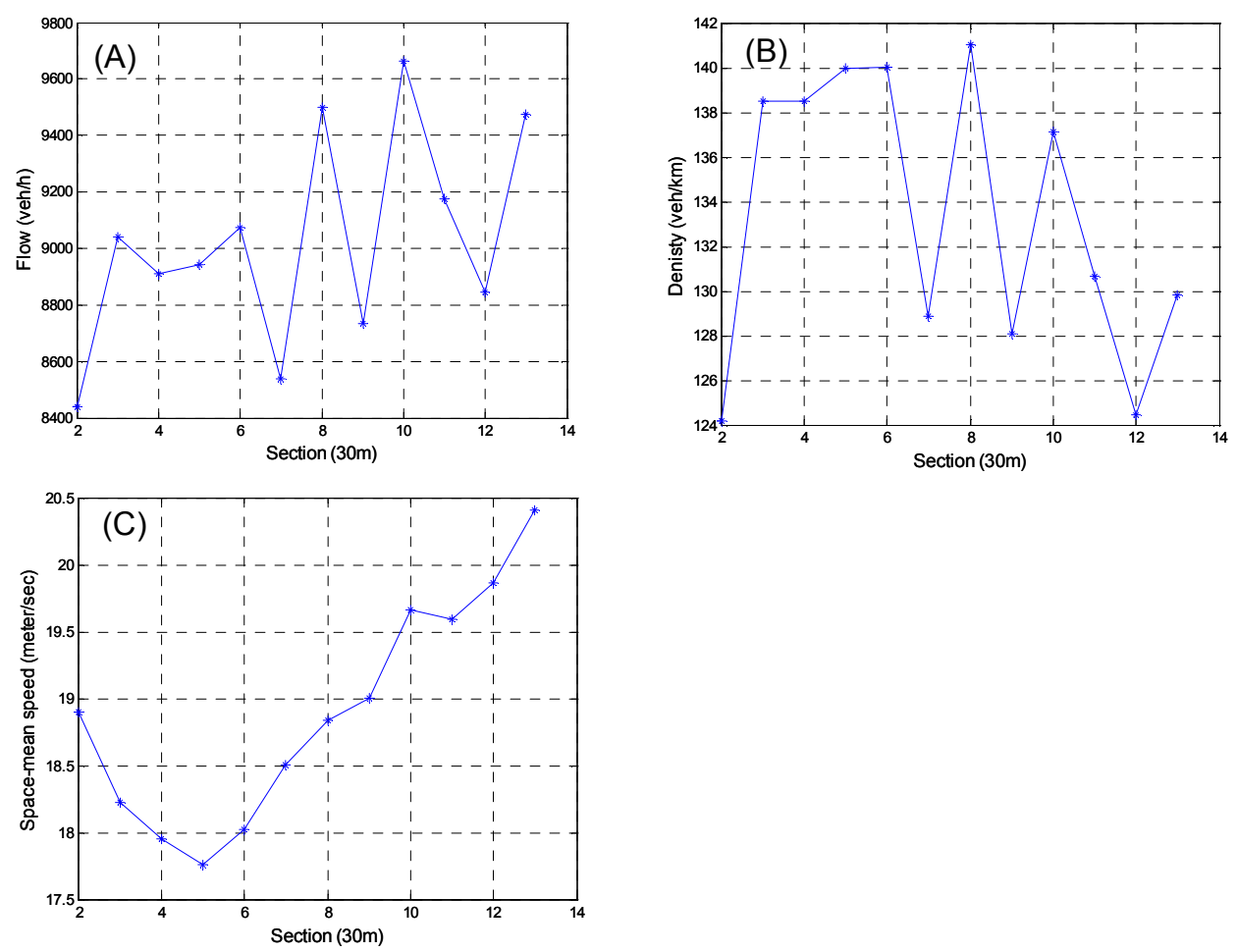

FIGURE A-31: Region 3--Flow, Density and Space-mean speed vs Freeway section on I405NB
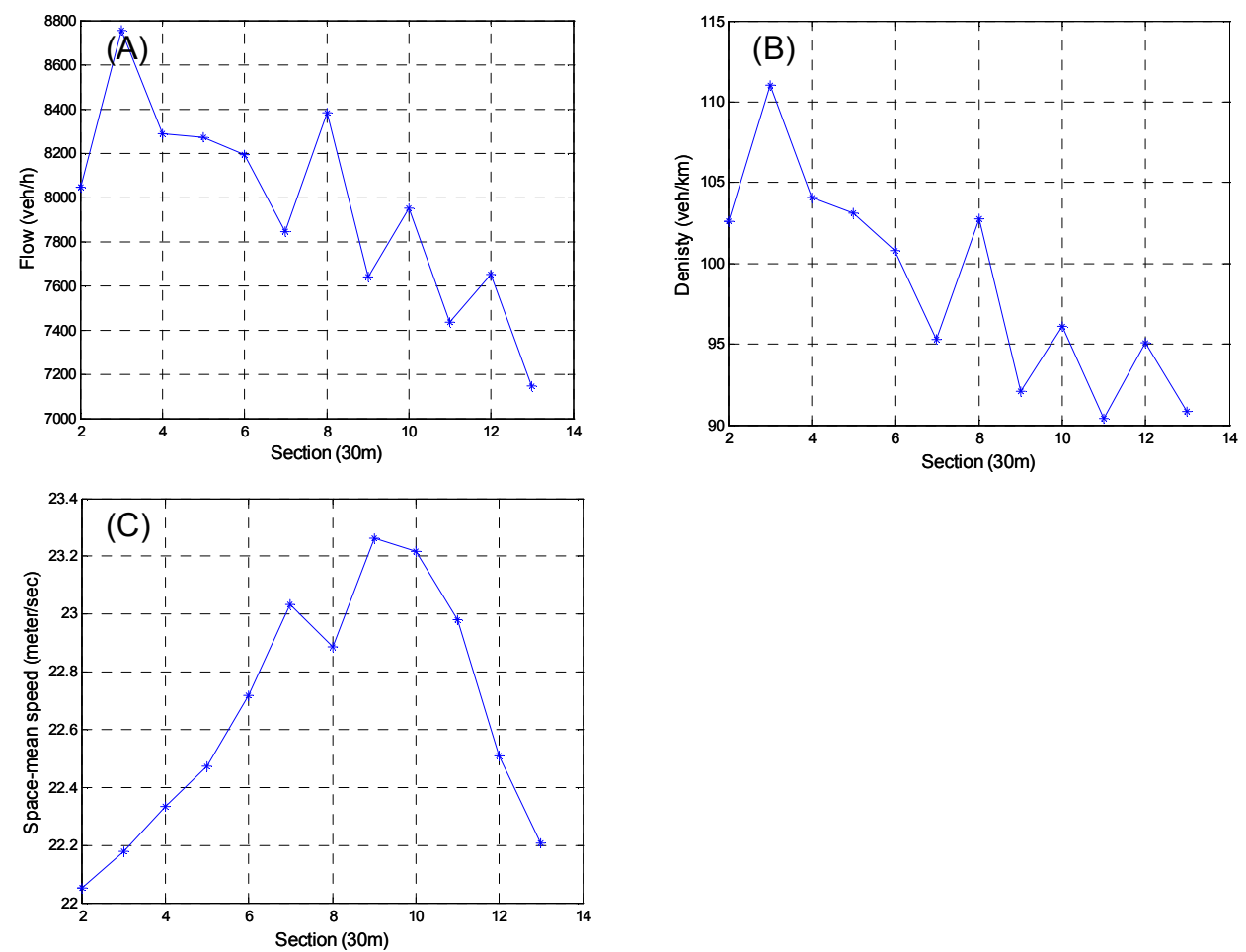

FIGURE A-32: Region 4--Flow, Density and Space-mean speed vs Freeway section on I405NB 

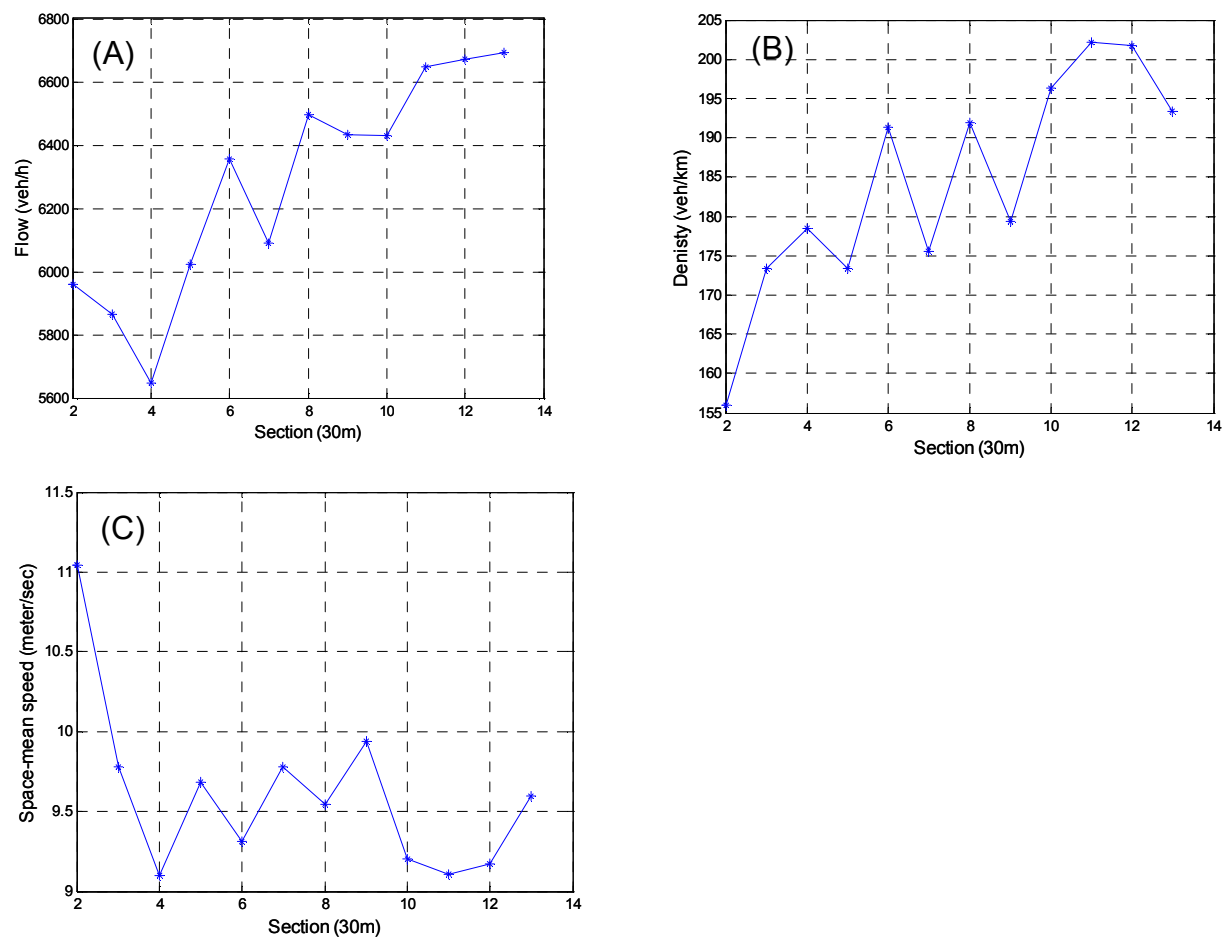

FIGURE A-33: Region 5--Flow, Density and Space-mean speed vs Freeway section on I405NB
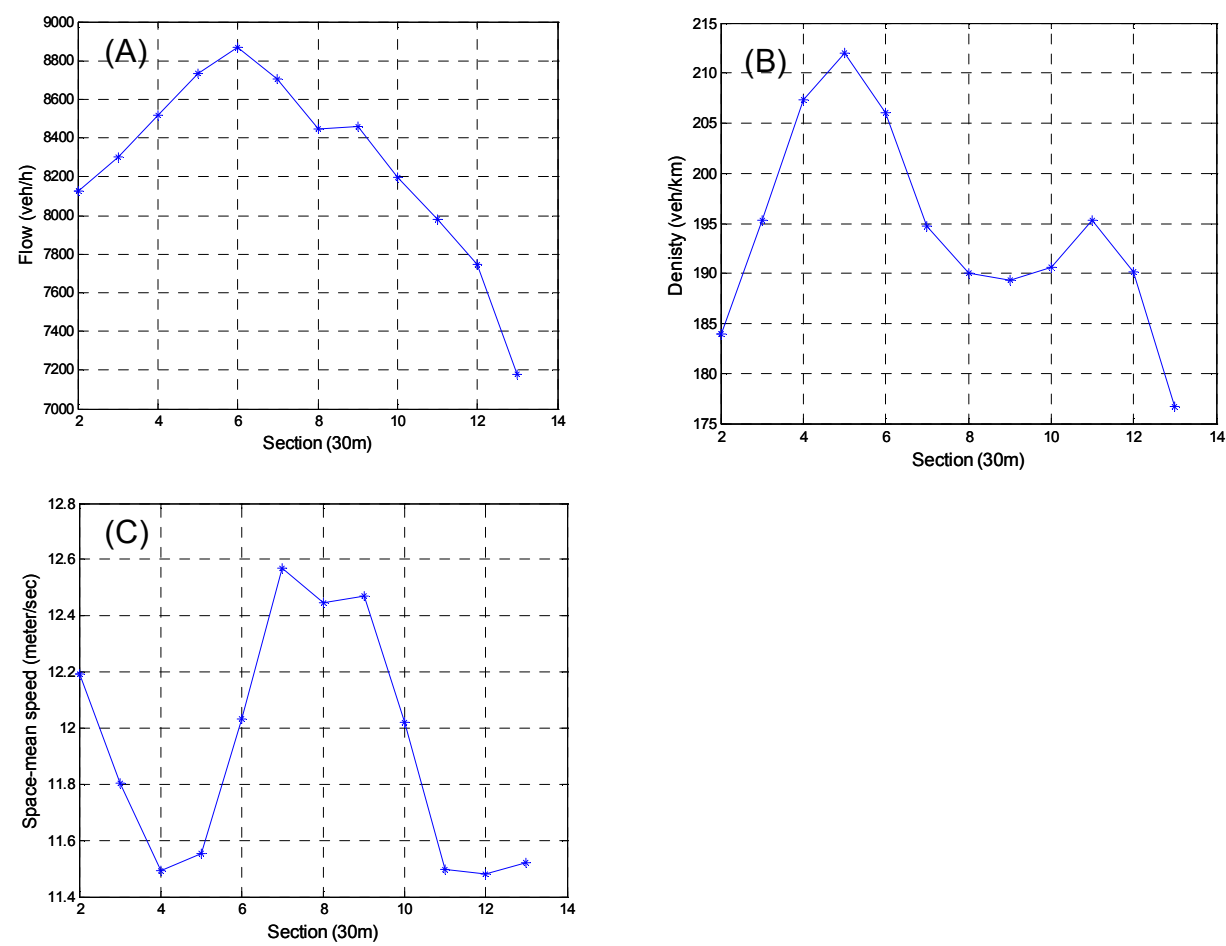

FIGURE A-34: Region 6--Flow, Density and Space-mean speed vs Freeway section on I405NB 

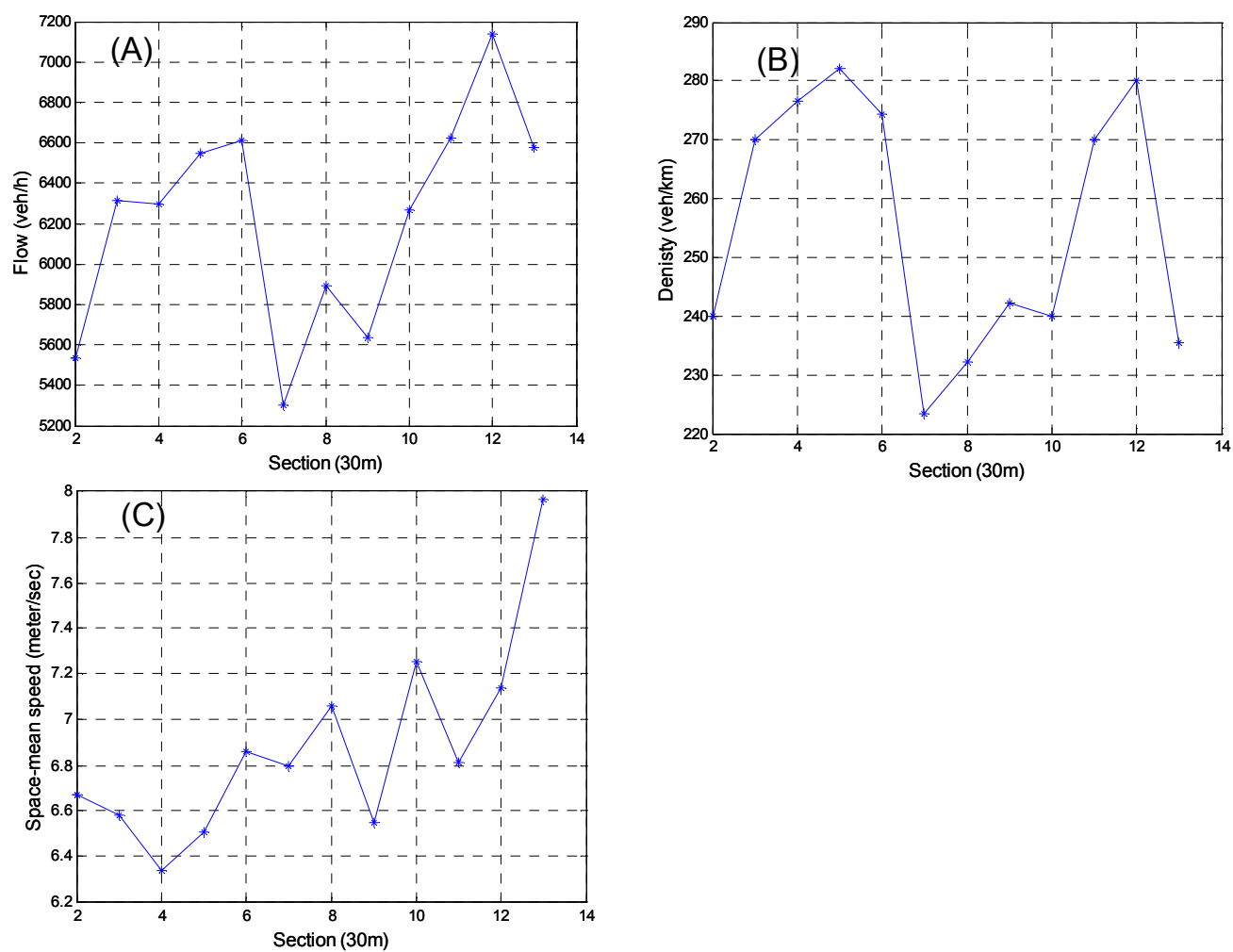

FIGURE A-35: Region 7--Flow, Density and Space-mean speed vs Freeway section on I405NB

\section{Results:}

The figures from B-29 to B-34 show the changes of flow, density and speed over sections, but no appropriate explanation for the results can be given for now. The most differences lie between microscopic data and loop detector data. Transition durations obtained from loop detectors range from four minutes up to 11 minutes, while transition durations obtained from microscopic trajectory data are only less than one minute. This finding is consistent with the analysis results from Dutch microscopic data.

Figure B-35 attempts to find the transition difference in different lanes. For this purpose, a simple Chi-square hypothesis is given as below:

\section{(A)}

Hypothesis test on transition durations

$\mathrm{H} 0$ : Transition duration on the left lane = transition duration on the right lane

H1: There is a significant difference between the transition durations on the left and right lanes. Chi-Square test: 11.5 points fall into the left plane, and 9.5 points fall into the right plane. Chi Square $=0.190$ and $\mathrm{P}$-value $=0.65$.

Therefore, H0 is accepted. 
(B)

Hypothesis test on transition rate

H0: Transition duration on the left lane $=$ transition duration on the right lane

H1: There is a significant difference between the transition durations on the left and right lanes

Chi-Square test: 7.5 points fall into the left plane, and 16.5 points fall into the right plane. Chi

Square $=3.375$ and P-value $=0.06$.

Therefore, $\mathrm{H} 1$ is accepted.

[Average transition from the left to the right]

$=[34.233 .837 .531 .67](\mathrm{sec})$

[Average transition rate from the left to the right]

$=\left[\begin{array}{llll}2.63 & 2.29 & 2.08 & 1.87\end{array}\right]\left(\right.$ meter $\left./ \sec ^{\wedge} 2\right)$

\section{Findings:}

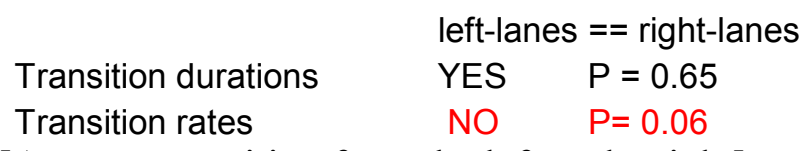

[Average transition from the left to the right]

$=[34.233 .837 .531 .67](\mathrm{sec})$

[Average transition rate from the left to the right]

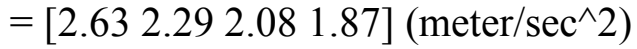

According to the above result, it is clearly shown that the transition durations have no significant difference between lanes, but transition rates do under $90 \%$ confidence circumstances. The

transition rates decrease from the left lane to the right lane. 



\section{SO OTREC \\ AND EDUCATION CONSORTIUM}

P.O. Box 751

Portland, OR 97207

OTREC is dedicated to stimulating and conducting collaborative multi-disciplinary research on multi-modal surface transportation issues, educating a diverse array of current practitioners and future leaders in the transportation field, and encouraging implementation of relevant research results. 